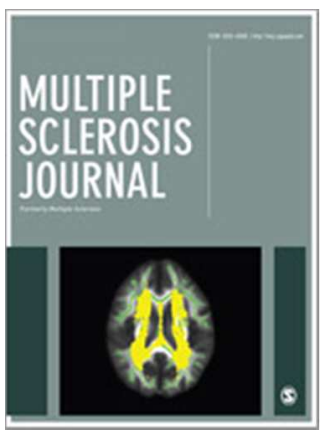

\title{
Data quality evaluation for observational multiple sclerosis registries
}

\begin{tabular}{|c|c|}
\hline Journal: & Multiple Sclerosis Journal \\
\hline Manuscript ID & MSJ-16-0400.R1 \\
\hline Manuscript Type: & Original Research Paper \\
\hline Date Submitted by the Author: & $\mathrm{n} / \mathrm{a}$ \\
\hline Complete List of Authors: & $\begin{array}{l}\text { Kalincik, Tomas; University of Melbourne, Department of Medicine; Royal } \\
\text { Melbourne Hospital, Department of Neurology } \\
\text { Kuhle, Jens; University Hospital Basel, Neurology } \\
\text { Pucci, Eugenio; Hospital of Macerata, Neurology Department } \\
\text { Rojas, Juan; Italian Hospital of Buenos Aires, MS section } \\
\text { Tsolaki, Magda; Aristotle University of Thessaloniki, 3rd Department of } \\
\text { Neurology } \\
\text { Sirbu, Carmen-Adella; Central Clinical Emergency Military Hospital, } \\
\text { Department of Neurology } \\
\text { Slee, Mark; Flinders Medical Centre, Department of Neurology } \\
\text { Butzkueven, Helmut; University of Melbourne, Department of Medicine; } \\
\text { Royal Melbourne Hospital, Department of Neurology }\end{array}$ \\
\hline Keywords: & Epidemiology, Multiple sclerosis \\
\hline Abstract: & $\begin{array}{l}\text { Objective } \\
\text { Objective and reproducible evaluation of data quality is of paramount } \\
\text { importance for studies of 'real-world' observational data. Here we } \\
\text { summarise a standardised data quality, density and generalisability process } \\
\text { implemented by MSBase, a global multiple sclerosis (MS) cohort study. } \\
\text { Methods } \\
\text { Error rate, data density score and generalisability score were developed } \\
\text { using all 35,869 patients enrolled in MSBase as of November } 2015 \text {. The } \\
\text { data density score was calculated across six domains (follow-up, } \\
\text { demography, visits, MS relapses, paraclinical data, therapy) and } \\
\text { emphasised data completeness. The error rate evaluated syntactic } \\
\text { accuracy and consistency of data. The generalisability score evaluated } \\
\text { believability of the demographic and treatment information. Correlations } \\
\text { among the three scores and the number of patients per centre were } \\
\text { evaluated. } \\
\text { Results }\end{array}$ \\
\hline
\end{tabular}


Errors were identified at the median rate of 3 per 100 patient-years. The generalisability score indicated the samples' representativeness of the known MS epidemiology. Moderate correlation between the density and generalisability scores $(\rho=0.58)$ and a weak correlation between the error rate and the other two scores $(\rho=-0.32-0.33)$ were observed. The generalisability score was strongly correlated with centre size $(\rho=0.79)$. Conclusion

The implemented scores enable objective evaluation of the quality of observational MS data, with an impact on the design of future analyses.

\section{SCHOLARONE ${ }^{\text {in }}$}

Manuscripts 


\section{Title page}

Title

Data quality evaluation for observational multiple sclerosis registries

\section{Authors and affiliations}

Tomas Kalincik; Department of Medicine, University of Melbourne, Melbourne, Australia; and Department of Neurology, Royal Melbourne Hospital, Melbourne, Australia

Jens Kuhle; Neurology, Departments of Medicine, Biomedicine and Clinical Research, University Hospital Basel, Basel, Switzerland

Eugenio Pucci; Neurology Unit, ASUR Marche AV3, Macerata, Italy

Juan Ignacio Rojas; Hospital Italiano, Buenos Aires, Argentina

Magda Tsolaki; 3rd Department of Neurology, G. Papanicolaou Hospital, Aristotle University of Thessaloniki, Thessaloniki, Greece

Carmen-Adella Sirbu; Central Military Emergency University Hospital, Bucharest, Romania

Mark Slee; Flinders University and Medical Centre, Adelaide, Australia

Helmut Butzkueven; Department of Medicine, University of Melbourne, Melbourne, Australia; Department of Neurology, Royal Melbourne Hospital, Melbourne, Australia; and Department of Neurology, Box Hill Hospital, Monash University, Melbourne, Australia

on behalf of the MSBase Scientific Leadership Group and MSBase Study Group*

*The list of the Scientific Leadership Group members and the MSBase Study Group members is provided in the online supplement.

Corresponding author: Tomas Kalincik; L4 Centre, Melbourne Brain Centre at Royal Melbourne Hospital, Grattan St, Parkville VIC 3050, Australia; Tel: +61 39342 4404, Fax: +61 39349 5997; email: tomas.kalincik@unimelb.edu.au

\section{Keywords}

multiple sclerosis, generalisability, representativeness, quality, data reporting, cohort study

\section{Word count}

Main text: 3173

Abstract: 200

References: 24 
Abstract

Objective

Objective and reproducible evaluation of data quality is of paramount importance for studies of 'real-world' observational data. Here we summarise a standardised data quality, density and generalisability process implemented by MSBase, a global multiple sclerosis (MS) cohort study.

\section{Methods}

Error rate, data density score and generalisability score were developed using all 35,869 patients enrolled in MSBase as of November 2015. The data density score was calculated across six domains (follow-up, demography, visits, MS relapses, paraclinical data, therapy) and emphasised data completeness. The error rate evaluated syntactic accuracy and consistency of data. The generalisability score evaluated believability of the demographic and treatment information. Correlations among the three scores and the number of patients per centre were evaluated.

\section{Results}

Errors were identified at the median rate of 3 per 100 patient-years. The generalisability score indicated the samples' representativeness of the known MS epidemiology. Moderate correlation between the density and generalisability scores $(\rho=0.58)$ and a weak correlation between the error rate and the other two scores $(\rho=-$ 0.32-0.33) were observed. The generalisability score was strongly correlated with centre size $(\rho=0.79)$.

\section{Conclusion}

The implemented scores enable objective evaluation of the quality of observational MS data, with an impact on the design of future analyses. 


\section{Main text}

\section{Introduction}

Observational data play an important role in the multiple sclerosis (MS) research. While traditionally utilised in epidemiology and the research of risk factors, observational cohorts have become a valuable source of information for the studies of treatment effectiveness and safety, disease management and outcomes. ${ }^{1}$ In particular, pragmatic trials utilising sophisticated analytical methodology, which have been known in diabetology and cardiology for more than two decades ${ }^{2,3}$ are now gaining momentum in neuroimmunology. ${ }^{4-6}$

The evidence generated from observational data carries two important limitations: residual bias (for review see ${ }^{7}$ ) and variable data quality. In MS research, the latter has yet to be addressed in a systematic way. The National Institutes of Health has emphasised data quality and included it as a review criterion. ${ }^{8}$ A Data Quality Collaborative was established in order to address the problems with observational data quality and has published recommendations comprising descriptions of data capture, processing, data elements and analysis-specific data elements. ${ }^{9}$

MSBase is a large, global observational cohort study, whose mission is to further the knowledge of MS epidemiology, aetiology and management and to generate evidence that will lead to improved outcomes of patients with this disease. ${ }^{10} \mathrm{We}$ have developed and implemented an automated data quality, density and generalisability process aiming at providing standardised, transparent, reproducible and highly granular information about the published data. The process will facilitate further improvement of data quality in MSBase and its collaborating centres. It will also enable quality-driven selection of research data and will enhance quality and generalisability of the generated evidence. Here we provide a detailed overview of 
the MSBase data quality, density and generalisability process and demonstrate its translational potential.

\section{Materials and Methods}

\section{Ethics}

MSBase (registered with WHO ICTRP, ID ACTRN12605000455662) was approved by the Melbourne Health Human Research Ethics Committee ${ }^{10}$ and the local ethics committees in all participating centres (or exemptions were granted, according to local laws and regulations). Enrolled patients provided written informed consent as required.

\section{Participants and definitions}

The data quality and generalisability scores were developed using the information from all 35,869 patients diagnosed with MS or clinically isolated syndrome represented in MSBase as of November 2015.

The prospective follow-up period was defined as the time between the first and last recorded disability scores. Disability was quantified by treating neurologists, using Expanded Disability Status Scale (EDSS). In order to minimise the EDSS inter-rater error, online Neurostatus certification was required at each participating centre (www.neurostatus.net). Relapses were defined as the occurrence of new symptoms, or exacerbation of existing symptoms, persisting for at least 24 hours, in the absence of concurrent illness or fever, and occurring at least 30 days after a previous relapse. ${ }^{11}$ Confirmation by increased EDSS was not required. 
Quality, density and generalisability of the recorded patient information was evaluated separately at each MSBase centre, assessing four quality metrics: ${ }^{12}$

- completeness (the proportion of the critical variables with data not missing),

- syntactic accuracy (the proportion of the recorded variables with the values corresponding to their range),

- consistency (the proportion of variables congruent with other recorded variables), and

- believability (the proportion of entries which are regarded true and credible).

\section{Data density score}

Data density was assessed primarily with respect to completeness across six domains: cumulative follow-up, patient demography, clinical visits, MS relapses, paraclinical data, and disease modifying therapy (Figure 1). The overall data density score was calculated as the sum of the scores within the six domains. The weights were chosen empirically in order to reflect the emphasis of the previous studies conducted using the MSBase data - i.e. clinical descriptors of disease course and activity, and MS therapy.

\section{Cumulative follow-up}

Centres were awarded one point for every 1000 patient-years' worth of prospective clinical follow-up. To valorise the centres with patients followed prospectively from early after clinical disease onset, the proportion of patients with first EDSS visit recorded within the year following the first clinical presentation of MS was valued with up to 3 points. Recency of clinical follow-up was assessed by EDSS visits recorded within the preceding year and was valued with 1 point. 


\section{Patient demography}

Centres reporting $\geq 0.02$ pregnancies per female (corresponding to the $4^{\text {th }}$ centile of the non-zero values) were awarded a data density point, in order to mitigate underreporting of pregnancies.

\section{Clinical visits}

Density points were awarded for each visit recorded on average per patient, 1.3 points for the visits with recorded EDSS and 0.2 points for the visits with no EDSS available, which reflects the importance of the documented clinical information (administrative or phone visits were excluded). Higher coefficient of variance of the annual visit frequency indicates greater variability in the frequency of clinical followup. Therefore, its inverse value was used as the score for regular clinical follow-up. MS relapses

Mean annualised relapse rate per centre was rated with 6 (if 0.2-1.5 relapse/year $\left[10^{\text {th }}-75^{\text {th }}\right.$ centile]), 3 (if $0-0.2$ or $1.5-2.5$ relapses/year $\left[>10^{\text {th }}\right.$ or $75^{\text {th }}-85^{\text {th }}$ centile $]$ ) or 0 points (if 0 or $>2.5$ relapse/year $\left[0^{\text {th }}\right.$ or $>85^{\text {th }}$ centile]), based on the known epidemiology of MS relapses. ${ }^{13}$ The completeness of the relapse-related information was evaluated as follows: the proportion of relapses with the information about the symptoms (max 3 points), severity (max 1.5 points), acute treatment (max 2 points), degree of recovery (max 1.5 points), and in addition up to 1 point for the proportion of relapses with all the above information recorded.

\section{Paraclinical data}

The paraclinical domain consisted of the information about brain magnetic resonance imaging $(\mathrm{MRI})$ and cerebrospinal fluid: the mean annual frequency of $\mathrm{MRI}$ investigations (using square root transformation to mitigate its markedly skewed 
distribution), the proportions of MRl's with categorised information about contrastenhancing lesions (max 4 points), new hyperintense T2 lesions (max 3 points), hyperintense T2 lesions ( $\max 2$ points), the proportion of patients with a recorded lumbar puncture (max 0.5 point), result of the cerebrospinal fluid analysis (max 1.5 points), and presence/absence of IgG oligoclonal bands indicating intrathecal immunoglobulin synthesis (max 2 points).

Disease modifying therapy

The therapy domain consisted of the score for the proportion of patients with $\geq 5$-year prospective follow-up after the diagnosis of clinically definite MS in whom disease modifying therapy has been recorded (max 6 points when $\geq 60 \%$ patients treated [ $\geq 40^{\text {th }}$ centile]), and the proportion of patients with adverse events recorded ( $\max 3$ points when $\geq 2 \%\left[\geq 40^{\text {th }}\right.$ centile $]$ ).

\section{Error rate}

Data quality procedure assessed the recorded data at each centre for syntactic accuracy (i.e. the proportion of records which fall within the realistic range of the given variable) and consistency (i.e. counting incomplete or erroneous dates, dates of MS-related events inconsistent with the date of MS onset or birth date, contradictory entries etc.). Data quality was evaluated across all six domains (see above) and quantified as the number of identified errors per 100 patient-years. In order to minimise inflation of its value, error rate was only calculated for centres with $\geq 25$ patient-years of cumulative follow-up. 


\section{Generalisability score}

Generalisability score is indicative of data believability, which is defined as the proportion of entries that are regarded true and credible with respect to the known epidemiology of MS.

Information about demography and disease modifying therapy was used to calculate the generalisability score separately at each MSBase centre. Female-to-male ratio was quantified with $6(1.5-5.7: 1), 3(1-1.5: 1$ or $5.7-9: 1)$ or 0 points $(0-1: 1$ or $>9: 1){ }^{14}$ The proportions of patients with the different MS phenotypes were evaluated as follows: relapsing-remitting MS, 4 (60-80\%), 2 (15-20\% or $80-90 \%)$ or 0 points (0$50 \%$ or $>90 \%$ ); secondary progressive MS,1 points (if $20-35 \%$, otherwise 0 points); and primary progressive MS, 0.5 points (if $7-15 \%$, otherwise 0 points). ${ }^{15}$ The proportion of patients with $\geq 5$-year prospective follow-up after the diagnosis of clinically definite MS in whom any disease modifying therapy has been recorded was scored with up to 4 points (when $\geq 80 \%$ patients were treated). The proportion of the prospective follow-up time for which patients were treated with disease modifying therapies was awarded up to 2.7 points (max at $90 \%$ of time, for more than $90 \%$ of the follow-up time, 2.4 were awarded). For each disease modifying therapy represented at MSBase centre (max 14 therapies), 0.4 points were awarded.

\section{Data analysis}

The scores were calculated and the analysis was performed using the $\mathrm{R}$ package (version 3.1.0). ${ }^{16}$ The inferential statistics consisted of six tests of Spearman's rank correlation coefficients between the data error rate, data density score, generalisability score and the number of patients contributed to the MSBase cohort per centre. 


\section{Results}

In November 2015, the MSBase cohort consisted of 35,869 patients, representing 179,594 patients-years, and followed at 117 actively participating centres in 34 countries (see Supplementary Table 1). The median prospective follow-up was 3.4 years per patient, with the median interval between the visits with EDSS assessments being 8 months. The characteristics of the cohort are shown in Table 1. Distributions of the variables that informed this assessment are shown in Table 2. The list of individual variables used to calculate error rate and the density and generalisabiltiy scores for each centre is shown in Supplementary Table 2.

The error rate was calculated with the aim of quantifying syntactic accuracy and consistency of the information recorded by the MSBase centres. The median estimated error rate was 3 errors per 100 patient-years (interquartile range 1.9-8.7). Centres contributing greater sample to the MSBase cohort tended to record lower error rates $\left(\rho=-0.43, p=10^{-5}\right.$; see Figure 2).

Completeness of the data was quantified using the data density score. Of its six domains clinical visits and MS relapses contributed the largest weights to the overall score (31\% and 26\%, respectively). The data density score ranged from 1 to 103 points, with mean \pm standard deviation of $36 \pm 17$. Similarly, the data density score was positively associated with the size of the MSBase centres $\left(\rho=0.37, p=10^{-5}\right)$.

The purpose of the generalisability score was to quantify believability of the recorded information. We have estimated this as the representativeness of the patient cohort recorded at each of the MSBase centres with respect to the known epidemiology of 
MS and the use of disease modifying therapies. The generalisability score consisted of the information concerning patient demography, MS phenotypes and exposure to disease modifying therapy. It ranged from 0 to 21 points, with mean \pm standard deviation of $14 \pm 6$ and was strongly correlated with MSBase centre size $(\rho=0.79$, $\left.\mathrm{p}<10^{-16}\right)$.

The correlation of the error rate with the data density score $(\rho=-0.32, p=0.002)$ and the generalisability score $(\rho=-0.33, p=0.002)$ was only weak (see Figure 3$)$. The data density score and the generalisability score were correlated with a moderate strength $\left(\rho=0.58, p=10^{-11}\right)$.

Supplementary Figure shows error rates and the generalisability scores and a breakdown of the data density scores with its six domains for the individual MSBase centres. The majority of the centres reached error rates between 0 and 20 errors per 100 patient-years, with 7 centres reaching error rates of $<1$. While for the majority of the centres the representation of the score components was consistent with the contribution of the components to the overall score, a small number of centres included information from only a small number of patients and their scores were driven by the high granularity of the clinical visits data. These sub-cohorts typically represented treatment-specific cohorts with dense clinical follow-up (such as monthly EDSS in patients treated with natalizumab, which is a requisite of access to these therapies in some jurisdictions).

As an example of the implementation of the procedure aiming at maximising data quality, density and representativeness, we have applied the following criteria to model a patient sample from the MSBase cohort: maximum error rate of 1.6 per 100 patient-years, data density score $\geq 60$ (i.e. $\geq 58 \%$ of the maximum score), generalisability score $\geq 15$ (i.e. $\geq 71 \%$ of the maximum score) and number of patients 
contributed per centre $\geq 25$. The resulting sample consisted of 6327 patients $(71 \%$ female) from 3 centres (error rates $0.4,1.2,1.6$, data density scores $63,79,72$, and generalisability scores 19, 21, 16, and patients contributed per centre 1308, 3493, 1526, respectively), with 52,889 patient-years of prospective follow-up (mean followup 8.4 years per patient), the mean of 19 visits recorded per patient ( 2.3 visits per year, $95 \%$ of these including an EDSS score), and with $40 \%$ of the selected patients having a visit recorded within the previous year. The sample consisted of patients with the following MS phenotypes: relapsing-remitting $73 \%$, secondary progressive $10 \%$, primary progressive $4 \%$, progressive-relapsing $1 \%$, and clinically isolated syndrome $12 \%$. The annualised relapse rate reached 0.54 relapses per year, $91 \%$ of the relapse events contained information about relapse phenotype, and $6 \%$ of relapses had all information available (including phenotype, severity, therapy and recovery). In $45 \%$ of women a pregnancy was recorded. Of the selected patients, $70 \%$ have been exposed to disease modifying therapies with the mean on-treatment time being $56 \%$ of the prospective follow-up; 13 disease modifying therapies were represented. The recorded incidence of adverse events was 0.11 per patient-year. The mean frequency of brain MRI within the sample was 0.9 per year, with the entries containing the information about hyperintense T2 lesions, contrast-enhancing lesions or the number of new T2 lesions in $45 \%, 41 \%$ and $4 \%$, respectively. In $63 \%$ of these patients, a lumbar puncture was recorded, with the information about the cytology and biochemistry recorded in $61 \%$ and oligoclonal bands in $27 \%$ of the patients. The average frequency of data entry errors was 1.1 per 100 patient-years. 


\section{Discussion}

Using MSBase, a large global MS cohort study, we have developed and implemented a standardised data quality, density and generalisability process. The process quantifies completeness, accuracy, consistency and believability ${ }^{12}$ of demographic, clinical and paraclinical information with respect to the specific requirements of an observational MS cohort. The resulting metrics enable monitoring and improvement of the quality of the data recorded at the participating centres, and provide the basis for selection of high-quality representative data for future analyses.

Hidden confounders represent a major limitation of the studies using observational cohorts. ${ }^{1}$ These confounders often originate from the variability in the quality and density of the data recorded within multicentric observational datasets. The risk of hidden bias has prompted the development of recommendations for transparent reporting of data quality in distributed data networks. ${ }^{9}$ MSBase in its multiple publications of observational data has previously used data quality procedures (see Online Supplement 2 for a typical MSBase data quality procedure). ${ }^{17}$ However, there has been a need for a unified data quality, density and generalisability protocol, standardised across the MSBase centres, which would incorporate the recommendations of the Data Quality Collaborative, with the potential to inform similar procedures in other observational cohorts.

A methodological template for adopting the process quantifying data completeness, accuracy, consistency and believability was provided in the European Society of Thoracic Surgeons Database (in a setting of a cross-sectional registry). ${ }^{18}$ We have extended this concept to a global longitudinal observational cohort study. In a multicentric registry, such evaluation then becomes suitable for comparison of data quality among the contributing sites, as demonstrated in the Manchester 
Orthopaedic Database. ${ }^{19}$ Importantly, per-centre evaluation of data quality enables identification of error (mainly systematic, and to a lesser extent also random) and provides an invaluable opportunity for improving data quality. An example of this was provided by the data quality process implemented by the Perfusion Downunder Collaboration, collecting information about cardiopulmonary bypass from three regional centres in South Australia. ${ }^{20}$

Data quality and generalisability are closely related. For example, a Global Network's Maternal Newborn Health Registry (trial registration number NCT01073475) has implemented a performance metric in which data quality metrics (such as the proportion of enrolled subjects or the proportions of measured, estimated and missing birth weights) are combined with the generalisability metrics (such as gender ratio or ratio of stillbirths to early neonatal deaths) into a single composite score. ${ }^{21}$ We have chosen to separate the error rate, and data density and generalisability scores in order to allow for a greater flexibility of their implementation. This allows us to take into consideration the purpose of data acquisition in the individual centres with respect to the scope of the MSBase studies (e.g. generalisability may be of a relatively greater importance for studies of MS epidemiology than studies of a therapeutic intervention in a specific subpopulation). ${ }^{22}$ In fact, error rate was only weakly correlated with the data density and generalisability scores, and these are therefore likely to be complementary (for a more detailed discussion see ${ }^{1}$ ).

The data density and generalisability scores were moderately correlated, which is partly determined by a modest overlap in the included variables (i.e. the proportion of patients treated with immunomodulatory therapies). The associations between centre size and error rate or the data density score were only weak, which confirms that the scores are not biased against the smaller centres. It is also worth noting that 
the data contributed by MSBase centres with relatively larger patient populations were more likely to be of relatively higher generalisability and therefore more representative of the general MS population.

The new standardised MSBase data quality process is comparable to the data quality assessment for drug safety surveillance system proposed by the Observational Medical Outcomes Partnership, with the emphasis on the detection of data anomalies (represented by the error rate) and determining their cause (as captured by the data density score). ${ }^{23}$ Characterisation of the data source has been provided elsewhere. ${ }^{10}$ Thus the MSBase error rate and the density score address the issue of data plausibility and the generalisability score (together with the requirement of Neurostatus certification (www.neurostatus.net) for the purpose of disability evaluation at each active MSBase centre) partially addresses the issue of organisation. $^{24}$

However, in its present format the MSBase data quality process does not involve source data verification and unified, global auditing. This is primarily restricted by the prohibitive cost of source data verification at the scale of a large international collaborative initiative. Hence, the actual data fidelity can only be expected in those MSBase centres where high plausibility and good organisation have been observed. ${ }^{24}$ In addition, the limited scope of the audit trail implemented within the current MSBase data entry software (iMed) limits the ability to quantify the time lag between events and their entries. The above represent the opportunities for further improvement, namely establishing a unified auditing system and redeveloping the current MSBase audit trail. Because no normative base exists for longitudinal evaluation of MS data quality, ongoing audit of the long-term impact of the data quality process is essential. Further areas for improvement are bundled with 
implementation of real-time congruence assessment within the MSBase data entry system.

The standardised data quality process described above will be implemented as an automated MSBase process. MSBase will periodically provide feedback to the participating centres including detailed characteristics of their contributed cohorts, their overall ranking in data quality, density and generalisability, as well as the ranking in each of the data density score domains. The three scores will also be used to define cohorts above the minimum data quality, density and generalisability for inclusion in the future studies and, where applicable, to calculate weights used in statistical models. The flexibility allowed by the three separate scores and the six domains of the data density score will enable researchers to tailor these criteria with respect to the aims of the particular research studies. 


\section{Disclosure statement}

Authors did not have any conflict of interest relevant to the presented work. Tomas Kalincik has served on an advisory scientific board for Novartis, Merck and Biogen, has received conference travel support and/or speaker honoraria from WebMD Global, Novartis, Biogen, Sanofi, Genzyme, Teva, BioCSL and Merck and has received research support from Biogen.

Jens Kuhle's institution (University Hospital Basel) received in the last 3 years and used exclusively for research support: consulting fees from Novartis, Protagen AG; speaker fees from the Swiss MS Society, Biogen, Novartis, Roche, Genzyme; travel expenses from Merck Serono, Novartis; grants from ECTRIMS Research Fellowship Programme, University of Basel, Swiss MS Society, Swiss National Research Foundation, Bayer (Schweiz) AG, Genzyme, Novartis.

Eugenio Pucci served on scientific advisory boards for Merck Serono, Genzyme and Biogen; he has received honoraria and travel grants from Sanofi Aventis, UCB, Lundbeck, Novartis, Bayer Schering, Biogen, Merck Serono, Genzyme and Teva; he has received travel grants and equipment from "Associazione Marchigiana Sclerosi Multipla e altre malattie neurologiche". Juan Ignacio Rojas has received honoraria from Novartis as a scientific advisor. He has received travel grants and attended courses and conferences on behalf of Merck-Serono and Novartis.

Magda Tsolaki did not declare any competing interests.

Carmen-Adella Sirbu received speaking honoraria from Teva, and travel grants from Bayer-Schering and Teva.

Mark Slee has participated in, but not received honoraria for, advisory board activity for Biogen, MerckSerono, BayerSchering, Sanofi Aventis and Novartis.

Helmut Butzkueven has served on scientific advisory boards for Biogen, Novartis and Sanofi-Aventis and has received conference travel support from Novartis, Biogen and Sanofi Aventis. He serves on steering committees for trials conducted by Biogen and Novartis, and has received research support from Merck Serono, Novartis and Biogen. 


\section{References}

1. Kalincik T and Butzkueven $\mathrm{H}$. Observational data: understanding the real MS world. Mult Scler. Epub ahead of print 6 June 2016; DOI: $10.1177 / 1352458516653667$.

2. Ziff OJ, Lane DA, Samra M, et al. Safety and efficacy of digoxin: systematic review and meta-analysis of observational and controlled trial data. Bmj. 2015; 351: h4451.

3. Steineck I, Cederholm J, Eliasson B, et al. Insulin pump therapy, multiple daily injections, and cardiovascular mortality in 18,168 people with type 1 diabetes: observational study. Bmj. 2015; 350: h3234.

4. Busch RM, Love TE, Jehi LE, et al. Effect of invasive EEG monitoring on cognitive outcome after left temporal lobe epilepsy surgery. Neurology. 2015; 85: 1475-81.

5. Kalincik T, Horakova D, Spelman T, et al. Switch to natalizumab vs fingolimod in active relapsing-remitting multiple sclerosis. Ann Neurol. 2015; 77: 425-35.

6. Iaffaldano P, Lucisano G, Pozzilli $C$, et al. Fingolimod versus interferon beta/glatiramer acetate after natalizumab suspension in multiple sclerosis. Brain. 2015.

7. Sormani MP and Bruzzi P. Can we measure long-term treatment effects in multiple sclerosis? Nat Rev Neurol. 2015; 11: 176-82.

8. NIH Health Care Systems Research Collaboratory - Demonstration Projects for Pragmatic Clinical Trials Focusing on Multiple Chronic Conditions (UH2/UH3), http:/grants.nih.gov/grants/guide/rfa-files/RFA-RM-13-012.html; accessed 10/01/2016. 
9. Kahn MG, Brown JS, Chun AT, et al. Transparent reporting of data quality in distributed data networks. EGEMS (Washington, DC). 2015; 3: 1052.

10. Butzkueven H, Chapman J, Cristiano E, et al. MSBase: an international, online registry and platform for collaborative outcomes research in multiple sclerosis. Mult Scler. 2006; 12: 769-74.

11. Schumacker GA, Beebe G, Kibler RF, et al. Problems of Experimental Trials of Therapy in Multiple Sclerosis: Report by the Panel on the Evaluation of Experimental Trials of Therapy in Multiple Sclerosis. Annals of the New York Academy of Sciences. 1965; 122: 552-68.

12. Batini C, Cappiello C, Francalanci C and Maurino A. Methodologies for data quality assessment and improvement. ACM Comput Surv. 2009: 1-52.

13. Kalincik T. Multiple Sclerosis Relapses: Epidemiology, Outcomes and Management. A Systematic Review. Neuroepidemiology. 2015; 44: 199-214.

14. Kalincik T, Vivek V, Jokubaitis V, et al. Sex as a determinant of relapse incidence and progressive course of multiple sclerosis. Brain. 2013; 136: 3609-17.

15. Pugliatti M, Rosati G, Carton $\mathrm{H}$, et al. The epidemiology of multiple sclerosis in Europe. Eur J Neurol. 2006; 13: 700-22.

16. R Development Core Team. R: A language and environment for statistical computing. Vienna, Austria: R Foundation for Statistical Computing; 2011.

17. Rossman IT and Cohen JA. Multiple sclerosis: Switching sides--fingolimod versus injectable MS therapies. Nat Rev Neurol. 2015; 11: 316-7.

18. Salati M, Brunelli A, Dahan M, Rocco G, Van Raemdonck DE and Varela G. Task-independent metrics to assess the data quality of medical registries using the European Society of Thoracic Surgeons (ESTS) Database. European journal of 
cardio-thoracic surgery : official journal of the European Association for Cardiothoracic Surgery. 2011; 40: 91-8.

19. Ricketts D, Newey M, Patterson M, Hitchin D and Fowler S. Markers of data quality in computer audit: the Manchester Orthopaedic Database. Annals of the Royal College of Surgeons of England. 1993; 75: 393-6.

20. Tuble SC. Perfusion Downunder Collaboration Database--data quality assurance: towards a high quality clinical database. The journal of extra-corporeal technology. 2011; 43: P44-51.

21. Goudar SS, Stolka KB, Koso-Thomas M, et al. Data quality monitoring and performance metrics of a prospective, population-based observational study of maternal and newborn health in low resource settings. Reproductive health. 2015; 12 Suppl 2: S2.

22. Sorensen HT, Sabroe S and Olsen J. A framework for evaluation of secondary data sources for epidemiological research. International journal of epidemiology. 1996; 25: 435-42.

23. Hartzema AG, Reich CG, Ryan PB, et al. Managing data quality for a drug safety surveillance system. Drug safety. 2013; 36 Suppl 1: S49-58.

24. Stausberg J, Nonnemacher M, Weiland D, Antony G and Neuhauser M. Management of data quality--development of a computer-mediated guideline. Studies in health technology and informatics. 2006; 124: 477-82. 


\author{
Tables \\ Table 1 \\ Characteristics of the MSBase cohort \\ at inclusion at last EDSS visit \\ patients, number (female, \%) $\quad 35,869(71 \%)$ \\ age, years $^{\ddagger} \quad 38 \pm 12 \quad 43 \pm 12$ \\ follow-up duration, years* $\quad 3.4(0.3,8.0)$ \\ time from MS onset, years* $\quad 3.6(0.8,10) \quad 9.9(4.6,17)$ \\ disability, EDSS step* $\quad 2(1.5,3.5) \quad 2.5(1.5,5)$ \\ annualised relapse rate* $\quad 0.34(0,0.86)$ \\ disease course, number (\%) \\ missing \\ $1882(5.2 \%)$ \\ $0(0 \%)$ \\ clinically isolated syndrome \\ $7979(22.2 \%)$ \\ 3066 (9.4\%) \\ relapsing-remitting MS \\ $21,600(60.2 \%) \quad 23,541(72.0 \%)$ \\ secondary progressive MS \\ $2183(6.1 \%)$ \\ $3874(11.8 \%)$ \\ primary progressive MS \\ $1825(5.1 \%)$ \\ $1622(5.0 \%)$ \\ progressive relapsing MS \\ $388(1.1 \%)$ \\ $603(1.8 \%)$ \\ visits per patient, number ${ }^{*}$ \\ $5(2,13)$ \\ visit frequency (with EDSS), per year ${ }^{*}$ \\ $1.5(1.4,2.6)$ \\ patients treated with disease modifying therapy during follow-up, number (\%)
any therapy
$22,097(61.6 \%)$
interferon $\beta-1 \mathrm{a}, \mathrm{IM}$
$6137(17.1 \%)$
interferon $\beta-1 \mathrm{a}, \mathrm{SC}$
$7556(21.1 \%)$
interferon $\beta-1 b$
$5199(14.5 \%)$
glatiramer acetate
$5568(15.5 \%)$
teriflunomide
$601(1.7 \%)$ 


$\begin{array}{lr}\text { dimethyl fumarate } & 815(2.3 \%) \\ \text { fingolimod } & 3497(9.7 \%) \\ \text { siponimod } & 3\left(10^{-5} \%\right) \\ \text { cladribine } & 68(0.2 \%) \\ \text { natalizumab } & 3304(9.2 \%) \\ \text { alemtuzumab } & 29(0.1 \%) \\ \text { rituximab } & 78(0.2 \%) \\ \text { mitoxantrone } & 1126(3.1 \%) \\ \text { autologous hematopoietic stem cell therapy } & 19(0.1 \%) \\ \text { enrolled in randomised clinical trials } & 142(0.4 \%) \\ \text { EDSS, Expanded Disability Status Scale } & 26 \%(0 \%, 92 \%) \\ \text { proportion of the follow-up time on disease modifying therapy* } & 3(1,6) \\ \text { total MRI scans per patient, number* } & 14,435(40 \%)\end{array}$


Table 2

\section{Distributions of the evaluated variables at MSBase centres}

patients, number

$105(19,345)$

female, $\%$

$69 \%(65 \%, 76 \%)$

cumulative follow-up, patient-years

$214(21,1494)$

patients with relapsing-remitting MS, \%

$82 \%(73 \%, 88 \%)$

patients with secondary progressive MS, \%

$6 \%(0 \%, 12 \%)$

patients with primary progressive MS, \%

$4 \%(0 \%, 8 \%)$

patients with a visit within the first year after disease onset, \%

$22 \%(11 \%, 37 \%)$

pregnancies per female

$0.07(0.00,0.19)$

recorded visits per patient, number

$4.8(2.3,10.6)$

coefficient of variance of the recorded visits

$0.69(0.55,0.90)$

annualised relapse rate

$0.79(0.47,1.33)$

relapses with recorded symptomatology, \%

$92 \%(74 \%, 100 \%)$

relapses with recorded severity, \%

$50 \%(20 \%, 91 \%)$

relapses with recorded therapy, \%

$52 \%(35 \%, 74 \%)$

relapses with recorded recovery , \%

$47 \%(24 \%, 83 \%)$

relapses with complete information, \%

$15 \%(4 \%, 42 \%)$

annual frequency of MRI

$1.06(0.49,1.98)$

MRl's with reported contrast-enhancing lesions, \%

$29 \%(6 \%, 60 \%)$

MRI's with reported new hyperintense T2 lesions, \%

$9 \%(1 \%, 26 \%)$

MRl's with reported hyperintense T2 lesions, \%

$55 \%(11 \%, 90 \%)$

patients with recorded lumbar puncture, $\%$

$33 \%(14 \%, 57 \%)$

patients with reported CSF information, \%

$18 \%(5 \%, 41 \%)$

patients with reported IgG oligoclonal band status, \%

$5 \%(0 \%, 16 \%)$

patients with $\geq 5$-year prospective follow-up and recorded therapy, $\%$ 
1

2

3

4

5

6

7

8

9

10

11

12

13

14

15

16

17

18

19

20

21

22

23

24

25

26

27

28

29

30

31

32

33

34

35

36

37

38

39

40

41

42

43

44

45

46

47

48

49

50

51

52

53

54

55

56

57

58

59

60
$76 \%(0 \%, 92 \%)$

patients with a reported adverse event, \%

$4 \%(0,16 \%)$

error rate (per 100 patient-years)

$3.0(1.9,8.7)$

The data are shown as median (quartiles).

CSF, cerebrospinal fluid; MRI, magnetic resonance imaging 


\section{Figure legends}

\section{Figure 1}

Contribution of patient characteristics to the data density score (and its domains), error rate and generalisability score.

\section{Figure 2}

Associations between the number of patients contributed to the global cohort per MSBase centre and the corresponding error rate $(\rho=-0.43)$, data density score $(\rho=0.37)$ and generalisaibility score $(\rho=0.79)$.

\section{Figure 3}

Associations among error rate, data density score and generalisability score. Circle size indicates the size of the cohort contributed by centre. Weak associations between error rate and the data density score $(\rho=-0.32)$ and the generalisability score $(\rho=-0.33)$, and moderate association between the data density score and the generalisability score $(\rho=0.58)$ were observed.

\section{Online supplementary material Online Supplement 1 MSBase Scientific Leadership Group, co-investigators and contributors}

\section{Online Supplement 2}

A typical data quality assessment applied in a number of previous MSBase studies

\section{Supplementary Table 1}

Number of participating patients per centre

\section{Supplementary Table 2}


Individual variables used to calculate error rate, the data density score and the generalisabiltiy score for each MSBase centre

\section{Supplementary Figure}

Error rate, data density score (and its components) and generalisability score calculated for each MSBase centre. 
female-to-male ratio

reported prevalence of MS phenotypes

patients treated

proportion of recorded time on treatment

therapies represented

Contribution of patient characteristics to the data density score (and its domains), error rate and generalisability score.

Figure 1 $480 \times 868 \mathrm{~mm}(120 \times 120 \mathrm{DPI})$ 

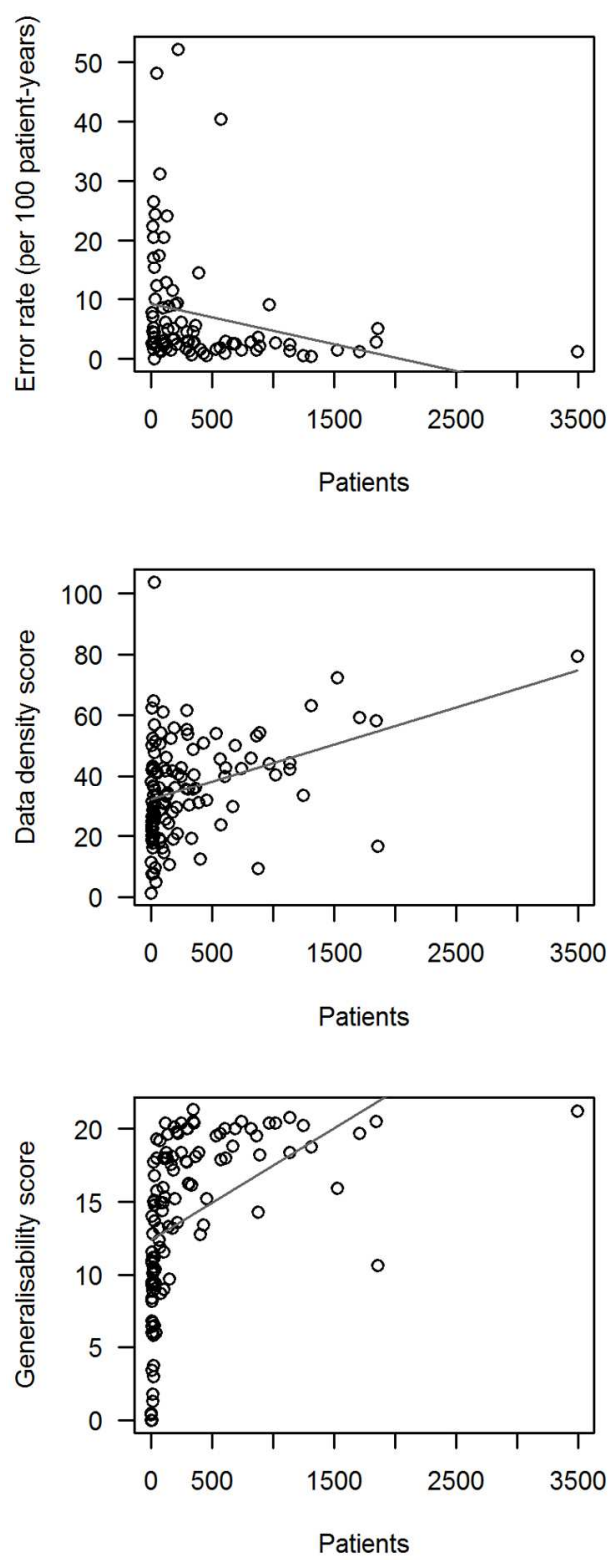

Associations between the number of patients contributed to the global cohort per MSBase centre and the corresponding error rate $(\rho=-0.43)$, data density score $(\rho=0.37)$ and generalisaibility score $(\rho=0.79)$. Figure 2

$69 \times 166 \mathrm{~mm}(300 \times 300 \mathrm{DPI})$ 


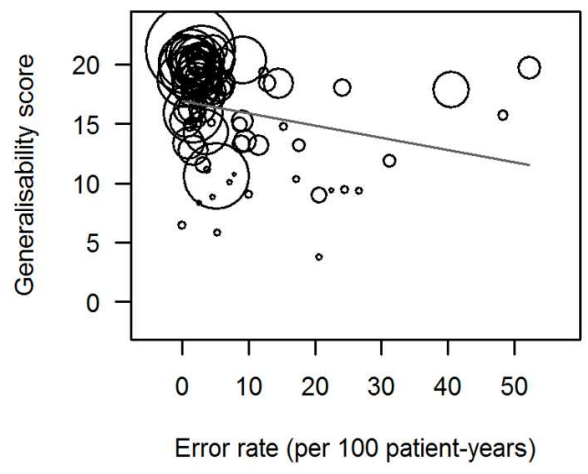

Associations among error rate, data density score and generalisability score. Circle size indicates the size of the cohort contributed by centre. Weak associations between error rate and the data density score ( $\rho=-$ $0.32)$ and the generalisability score $(\rho=-0.33)$, and moderate association between the data density score and the generalisability score $(\rho=0.58)$ were observed. Figure 3 $69 \times 170 \mathrm{~mm}(300 \times 300 \mathrm{DPI})$ 
MSBase Scientific Leadership Group

Dr Raed Alroughani, Dr Cavit Boz, Dr Helmut Butzkueven, Dr Edgardo Cristiano, Dr Francois Grand'Maison, Dr Orla Gray, Ms Jodi Haartsen, Dr Dana Horakova, Dr Raymond Hupperts, Dr Guillermo Izquierdo, Dr Tomas Kalincik, Dr Ludwig Kappos, Dr Ilya Kister, Dr Thomas Leist, Dr Sara Eichau Madueno, Dr Liliana Patrucco, Dr Maria Pia Sormani, Dr Maria Trojano, Dr Freek Verheul.

\section{MSBase study group co-investigators and contributors}

From CIREN, Havana, Cuba, Dr Jose Antonio Cabrera-Gomez.

From MS Clinic, Hopital Tenon, Paris, France, Dr Etienne Roullet.

From University Hospital Nijmegen, Nijmegen, Netherlands, Dr Cees Zwanikken.

From Francicus Ziekenhuis, Roosendaal, Netherlands, Dr Leontien Den Braber-Moerland.

From FLENI, Buenos Aires, Argentina, Dr Marcela Fiol.

From Hospital Fernandez, Capital Federal, Argentina, Dr Norma Deri.

From Hospital de Clinical, Buenos Aires, Argentina, Dr Alejandro Caride.

From Clinica Modelo de Lanus, Lanus, Argentina, Dr Alfredo Firstenfeld.

From Instituto Rodriguez Alfici, Mendoza, Argentina, Dr Alberto Rodriguez Alfici.

From Institute of Neuroscience Buenos Aires, Buenos Aires, Argentina, Dr Maria Laura Saladino.

From Instituto de Neurociencias Cordoba, Cordoba, Argentina, Dr Elizabeth Alejandra Bacile.

From Consultorio Privado, Capital Federal, Argentina, Dr Aldo Savino.

From Hospital Italiano, Buenos Aires, Argentina, Dr Edgardo Cristiano.

From HIGA Gral. San Martin La Plata, La Plata, Argentina, Dr Vetere Santiago.

From Sanatorio Allende, Cordoba, Argentina, Dr Carlos Vrech.

From Sir Charles Gairdner Hospital, Perth, Australia, Dr Allan Kermode.

From Brain and Mind Centre, Sydney, Australia, Dr Michael Barnett.

From University of Melbourne, Melbourne, Australia, Dr Tim Spelman, Dr Vilija Jokubaitis.

From Royal North Shore Hospital, Sydney, Australia, Dr John Parratt.

From University Newcastle, Newcastle, Australia, Dr Jeannette Lechner-Scott.

From Geelong Hospital, Geelong, Australia, Dr Cameron Shaw.

From St Vincents Hospital, Melbourne, Australia, Dr Neil Shuey.

From Monash Medical Centre, Melbourne, Australia, Dr Ernest Butler.

From Liverpool Hospital, Sydney, Australia, Dr Suzanne Hodgkinson.

From Box Hill Hospital, Melbourne, Australia, Ms Jodi Haartsen.

From Westmead Hospital, Sydney, Australia, Dr Steve Vucic.

From Royal Brisbane and Women's Hospital, Spring Hill, Australia, Dr Pamela McCombe.

From Royal Hobart Hospital, Hobart, Australia, Dr Michael Dreyer.

From The Alfred, Melbourne, Australia, Dr Olga Skibina.

From Austin Health, Melbourne, Australia, Dr Richard Macdonell.

From Macquarie University Hospital, Sydney, Australia, Dr Ik Lin Tan.

From Concord Repatriation General Hospital, Concord West, Australia, Dr Todd Hardy.

From Royal Brisbane and Women's Hospital, Brisbane, Australia, Dr Pamela McCombe.

From Cliniques Universitaires Saint-Luc, Brussels, Belgium, Dr Vincent Van Pesch.

From Hospital Ecoville, Curitiba, Brazil, Dr Walter Oleschko Arruda.

From Universidade Metropolitana de Santos, Santos, Brazil, Dr Yara Fragoso.

From CSSS Saint-Jérôme, Saint-Jerome, Canada, Dr Julie Prevost.

From Jewish General Hospital, Montreal, Canada, Dr Fraser Moore.

From Hopital Notre Dame, Montreal, Canada, Dr Pierre Duquette, Dr Marc Girard, Dr Alexandre Prat.

From CHRTR, Trois-Rivieres, Canada, Dr Stephane Charest.

From Centre de réadaptation déficience physique Chaudière-Appalache, Levis, Canada, Dr Pierre

Grammond.

From Neuro Rive-Sud, Greenfield Park, Canada, Dr Francois Grand'Maison.

From Universitatsspital Basel, Basel, Switzerland, Dr Ludwig Kappos.

From General University Hospital and Charles University in Prague, Prague, Czech Republic, Dr Eva Havrdova, Dr Dana Horakova.

From Nemocnice Jihlava, Jihlava, Czech Republic, Dr Radek Ampapa.

From Multiple Sclerosis Centre Kamillus-Klinik, Asbach, Germany, Dr Dieter Poehlau.

From Kommunehospitalet, Arhus C, Denmark, Dr Thor Petersen.

From Alexandria University Hospital, Alexandria, Egypt, Dr Karim Kotkata.

From Hospital Universitario Virgen de Valme, Seville, Spain, Dr Ricardo Fernandez Bolaños.

From Hospital Donostia, San Sebastián, Spain, Dr Javier Olascoaga.

From Hospital Universitario La Paz, Madrid, Spain, Dr Celia Oreja-Guevara. 
From Hospital Universitario Virgen Macarena, Sevilla, Spain, Dr Guillermo Izquierdo.

From Hospital Mancha Centro, Alcazar de San Juan, Spain, Dr Millan Pascual.

From Hospital de Galdakao-Usansolo, Galdakao, Spain, Dr Jose Luis Sanchez-Menoyo.

From Hospital Germans Trias i Pujol, Badalona, Spain, Dr Cristina Ramo.

From The Walton Centre for Neurology and Neurosurgery, Liverpool, United Kingdom, Dr Carolyn Young.

From Craigavon Area Hospital, Craigavon, United Kingdom, Dr Stella Hughes.

From Royal Victoria Hospital, Belfast, United Kingdom, Dr Gavin McDonnell.

From South East Trust, Belfast, United Kingdom, Dr Orla Gray.

From G. Papanicolaou Hospital, Thessaloniki, Greece, Dr Pavlos Myserlis.

From Instituto de Seguridad Social de Guatemala, Guatemala, Dr Alejandro Jose Diaz Jimenez.

From Veszprém Megyei Csolnoky Ferenc Kórház, Veszprem, Hungary, Dr Piroska Imre.

From Jahn Ferenc Teaching Hospital, Budapest, Hungary, Dr Csilla Rozsa, Dr Krisztian Kasa.

From Semmelweis University Budapest, Budapest, Hungary, Dr Magdolna Simo.

From University of Debrecen, Debrecen, Hungary, Dr Tunde Csepany.

From Péterfy Sandor Hospital, Budapest, Hungary, Dr Krisztina Kovacs.

From Josa András Hospital, Nyiregyhaza, Hungary, Dr Tunde Erdelyi.

From Petz A. County Hospital, Gyor, Hungary, Dr Gabor Rum.

From BAZ County Hospital, Miskolc, Hungary, Dr Attila Sas.

From Szent Imre Hospital, Budapest, Hungary, Dr Eniko Dobos.

From University of Pécs, Pecs, Hungary, Dr Anita Trauninger.

From Kaposi Mór Teaching Hospital, Kaposvar, Hungary, Dr Istvan Deme.

From University of Szeged, Szeged, Hungary, Dr Cecilia Rajda.

From Sheba Medical Center, Ramat Gan, Israel, Dr Joab Chapman.

From Hadassah University Hospital, Jerusalem, Israel, Dr Dimitrios Karussis.

From Assaf Harofeh Medical Center, Beer-Yaakov, Israel, Dr Shlomo Flechter.

From Bombay Hospital Institute of Medical Sciences, Mumbai, India, Dr Bhim Singhal.

From PGIMER, Chandigarh, India, Dr Dheeraj Khurana.

From Isfahan University of Medical Sciences, Isfahan, Iran, Dr Vahid Shaygannejad.

From Golestan, Ahvaz, Iran, Dr Seyed Aidin Sajedi.

From Azienda Ospedaliera San Luigi di Orbassano, Torino, Italy, Dr Antonio Bertolotto.

From University 'G. d'Annunzio', Chieti, Italy, Dr Alessandra Lugaresi, Dr Giovanna De Luca, Dr

Valeria Di Tommaso, Dr Daniela Travaglini, Dr Erika Pietrolongo, Dr Maria di loia, Dr Deborah Farina, Dr Luca Mancinelli.

From Azienda Sanitaria Unica Regionale Marche - AV3, Macerata, Italy, Dr Matteo Diamanti, Dr Elisabetta Cartechini.

From University of Bari, Bari, Italy, Dr Maria Trojano.

From University of Florence, Florence, Italy, Dr Maria Pia Amato.

From Universita, Napoli, Italy, Dr Pietro B Carrieri.

From C. Mondino National Neurological Institute, Pavia, Italy, Dr Roberto Bergamaschi.

From Ospedali Riuniti di Salerno, Salerno, Italy, Dr Gerardo luliano.

From University of Parma, Parma, Italy, Dr Franco Granella.

From Azienda Ospedaliera di Rilievo Nazionale San Giuseppe Moscati Avellino, Avellino, Italy, Dr Daniele Spitaleri.

From San Carlo Borromeo, Milan, Italy, Dr Simone Tonietti.

From Nuovo Ospedale Civile Sant'Agostino/Estense, Modena, Italy, Dr Patrizia Sola, Dr Diana

Ferraro, Dr Francesca Vitetta, Dr Anna Maria Simone.

From Amiri Hospital, Kuwait, Kuwait, Dr Raed Alroughani.

From Clinical Centar-Neurology, Skopje, Macedonia, Dr Vladimir Bojkovski.

From Clinic of Neurology Clinical Center, Skopje, Macedonia, Dr Tatjana Petkovska-Boskova.

From Mater Dei Hospital, Balzan, Malta, Dr Norbert Vella.

From Hospital Angeles de las Lomas. Instituto Mexicano de Neurociencias., Huixquilucan Estado de Mexico, Mexico, Dr Eli Skromne.

From Hospital Kuala Lumpour, Kuala Lumpur, Malaysia, Dr Joyce Pauline Joseph.

From Penang General Hospital, Penang, Malaysia, Dr Jyh Yung Hor.

From Hospital Sultanah Nora Ismail, Batu Pahat, Malaysia, Dr Chu Zhen Quek.

From University of Maiduguri Teaching Hospital, Maiduguri, Nigeria, Dr Yakub Nyandaiti.

From Zuyderland Ziekenhuis, Sittard, Netherlands, Dr Raymond Hupperts.

From Jeroen Bosch Ziekenhuis, Den Bosch, Netherlands, Dr Erik van Munster.

From Groene Hart Ziekenhuis, Gouda, Netherlands, Dr Freek Verheul. 
From Hospital São João, Porto, Portugal, Dr Maria Edite Rio. From Hospital Fernando Fonseca, Amadora, Portugal, Dr Ricardo Ginestal. From King Fahad Specialist Hospital-Dammam, Khobar, Saudi Arabia, Dr Talal Al-Harbi. From Dr Lutfi Kirdar Kartal Education and Research Hosp., Istanbul, Turkey, Dr Ulku Turk Boru. From KTU Medical Faculty Farabi Hospital, Trabzon, Turkey, Dr Cavit Boz. From 19 Mayis University, Medical Faculty, Samsun, Turkey, Dr Murat Terzi. From Cheng Hsin General Hospital, Taipei, Taiwan, China, Dr Kai Chen Wang.

From New York University Langone Medical Center, New York, United States, Dr llya Kister. From UT Southwestern, Dallas, United States, Dr Benjamin Greenberg. From Thomas Jefferson University, United States, Dr Donald McCarren.

\section{Administrative and technical support}

From the MSBase Administrations Dr Jill Byron, Ms Lisa Morgan and Ms Eloise Hinson. From Rodanotech, Geneva, Switzerland; Mr Samir Mechati, Mr Matthieu Corageoud, Mr Alexandre Bulla. 


\section{A typical MSBase data quality assessment}

2

3

4

- Duplicate patient records were removed.

- Centres with <10 patient record were excluded.

- Patients with missing date of birth were excluded.

- MS onset dates after the MSBase data extract date were removed.

- Patients with missing date of the first clinical presentation of MS were excluded.

- The dates of MS onset and the first recorded MS course were aligned.

- Patients with the age at onset outside the 0-100 range were excluded.

- A logical sequence of the MS courses (e.g. clinically isolated syndrome, relapsing-remitting MS, secondary progressive MS) was assured.

- Records of the initiation of the progressive MS prior to its clinical onset were excluded.

- Visits with missing visit date or the recorded date before the clinical MS onset or after the date of MSBase data extract were removed.

- EDSS scores outside the range of possible EDSS values were removed.

- Duplicate visits were merged.

- MS relapses with missing visit date or the recorded date after the date of MSBase data extract were removed.

- Duplicate MS relapses were merged.

- Relapses occurring within 30 days of each other were merged.

- Visits preceded by relapses were identified and time from the last relapse was calculated for each visit.

- Therapies were labelled as discontinued or continuing.

- Therapies with erroneous date entries were removed (e.g. commencement date > termination date, commencement after the MSBase data extract date, commencement of disease modifying therapy before the year 1980).

- MS disease modifying therapies were identified and labelled.

- Duplicate treatment entries were removed.

- Where multiple disease modifying therapies were recorded simultaneously, treatment end date of the previous therapy was imputed as the commencement date of the following therapy.

- Consecutive entries for certain disease modifying therapies were merged into a continuous treatment entry, given that the gap between the entries did not exceed 190 days for mitoxantrone, 365 days for cladribine, 90 days for other disease modifying therapies.

- The minimum duration of treatment effect was recorded as 190 days (mitoxantrone), 5 years (alemtuzumab) or 365 days (cladribine) from treatment commencement.

- MRI entries with missing or erroneous date (before the first clinical presentation of MS or after the MSBase data extract date) were removed.

- The terminology used for the McDonald criteria, Barkhoff criteria and the T2 or T1 contrastenhancing lesions was unified across the entries.

- Duplicate MRI entries were merged.

- Cerebrospinal fluid entries with missing or erroneous date (before the first clinical presentation of MS or after the MSBase data extract date) were removed.

- The terminology used to describe the result of the cerebrospinal fluid analysis was unified across the entries. 


\section{Number of participating patients per centre}

Centre

General University Hospital and Charles University, Prague, Czech Republic

Isfahan University of Medical Sciences, Isfahan, Iran

University 'G. d'Annunzio', Chieti, Italy

Hospital Universitario Virgen Macarena, Sevilla, Spain

University of Bari, Bari, Italy

Hopital Notre Dame, Montreal, Canada

University Hospital Nijmegen, Nijmegen, Netherlands

Amiri Hospital, Kuwait, Kuwait

Neuro Rive-Sud, Greenfield Park, Canada

19 Mayis University, Medical Faculty, Samsun, Turkey

KTU Medical Faculty Farabi Hospital, Trabzon, Turkey

Nuovo Ospedale Civile Sant'Agostino/Estense, Modena, Italy

Golestan, Ahvaz, Iran

Centre de réadaptation déficience physique Chaudière-Appalache, Levis, Canada

University of Melbourne, Melbourne, Australia

Box Hill Hospital, Box Hill, Australia

Zuyderland Ziekenhuis, Sittard, Netherlands

Hospital Universitario La Paz, Madrid, Spain

Cliniques Universitaires Saint-Luc, Brussels, Belgium

Ospedali Riuniti di Salerno, Salerno, Italy

University Newcastle, Newcastle, Australia

Bombay Hospital Institute of Medical Sciences, Mumbai, India

Azienda Sanitaria Unica Regionale Marche - AV3, Macerata, Italy

Kommunehospitalet, Arhus C, Denmark

C. Mondino National Neurological Institute, Pavia, Italy

Jeroen Bosch Ziekenhuis, Den Bosch, Netherlands

Brain and Mind Centre, Camperdown, Australia

Groene Hart Ziekenhuis, Gouda, Netherlands

Hospital Germans Trias i Pujol, Badalona, Spain

Flinders Medical Centre, Adelaide, Australia

Azienda Ospedaliera di Rilievo Nazionale San Giuseppe Moscati, Avellino, Italy

Royal Hobart Hospital, Hobart, Australia

Liverpool Hospital, Liverpool, Australia

Royal Brisbane and Women's Hospital, Spring Hill, Australia

Hospital Universitario Virgen de Valme, Seville, Spain

Hospital São João, Porto, Portugal

University of Parma, Parma, Italy

University of Florence, Florence, Italy

Hospital Italiano, Buenos Aires, Argentina

Westmead Hospital, Sydney, Australia

FLENI, Buenos Aires, Argentina

Monash Medical Centre, Melbourne, Australia

Craigavon Area Hospital, Craigavon, United Kingdom

Nemocnice Jihlava, Jihlava, Czech Republic

Hospital de Galdakao-Usansolo, Galdakao, Spain

Royal Victoria Hospital, Belfast, United Kingdom

Hopital Tenon, Paris, France

Institute of Neuroscience Buenos Aires, Buenos Aires, Argentina

CIREN, Havana, Cuba

Multiple Sclerosis Centre Kamillus-Klinik, Asbach, Germany

Francicus Ziekenhuis, Roosendaal, Netherlands

CSSS Saint-Jérôme, Saint-Jerome, Canada

The Alfred, Melbourne, Australia

Hospital Donostia, San Sebastián, Spain

Geelong Hospital, Geelong, Australia

Sir Charles Gairdner Hospital, Perth, Australia

St Vincents Hospital, Melbourne, Australia

Mater Dei Hospital, Balzan, Malta

Patients
3492
1857
1842
1705
1526
1308
1245
1136
1133
1021
969
885
874
863
820
738
686
665
609
603
566
565
528
451
429
402
391
362
348
345
345
329
308
303
300
292
291
282
246
245
221
216
207
196
186
179
176
174
161
149
140
138
130
123
116
114

Page 1 of 2 
Hospital Fernandez, Capital Federal, Argentina

New York University Langone Medical Center, New York, United States

Assaf Harofeh Medical Center, Beer-Yaakov, Israel 94

South East Trust, Belfast, United Kingdom $\quad 93$

Hospital Ecoville, Curitiba, Brazil $\quad 90$

PGIMER, Chandigarh, India

Jahn Ferenc Teaching Hospital, Budapest, Hungary 78

Hospital Angeles de las Lomas, Mexico, Mexico $\quad 78$

Jewish General Hospital, Montreal, Canada $\quad 73$

King Fahad Specialist Hospital-Dammam, Khobar, Saudi Arabia 67

Royal North Shore Hospital, Sydney, Australia $\quad 66$

Consultorio Privado, Capital Federal, Argentina 65

Austin Health, Heidelberg, Australia $\quad 45$

Clinic of Neurology Clinical Center, Skopje, Macedonia 43

Royal Brisbane and Women's Hospital, Brisbane, Australia 41

Thomas Jefferson University, United States $\quad 40$

Alexandria University Hospital, Alexandria, Egypt $\quad 30$

Semmelweis University Budapest, Budapest, Hungary 28

Instituto de Neurociencias Cordoba, Cordoba, Argentina 27

Central Clinical Emergency Military Hospital, Bucharest, Romania 26

HIGA Gral. San Martin La Plata, La Plata, Argentina 25

Concord Repatriation General Hospital, Concord West, Australia 25

University of Debrecen, Debrecen, Hungary 24

Péterfy Sandor Hospital, Budapest, Hungary 24

Sanatorio Allende, Cordoba, Argentina $\quad 22$

Clinica Modelo de Lanus, Lanus, Argentina 19

Universidade Metropolitana de Santos, Santos, Brazil 19

Veszprém Megyei Csolnoky Ferenc Kórház zrt., Veszprem, Hungary 19

Josa András Hospital, Nyiregyhaza, Hungary 19

Petz A. County Hospital, Gyor, Hungary 19

Hospital Kuala Lumpur, Kuala Lumpur, Malaysia 18

Macquarie University Hospital, Sydney, Australia 17

The Walton Centre for Neurology and Neurosurgery, Liverpool, United Kingdom 13

BAZ County Hospital, Miskolc, Hungary 13

Penang General Hospital, Penang, Malaysia 13

Instituto Rodriguez Alfici, Mendoza, Argentina 12

Szent Imre Hospital, Budapest, Hungary 12

Clinical Centar-Neurology, Skopje, Macedonia $\quad 12$

CHRTR, Trois-Rivieres, Canada 11

Hospital de Clinicas, Bbuenos Aires, Argentina 9

Instituto de Seguridad Social de Guatemala, Guatemala, Guatemala 9

Azienda Ospedaliera San Luigi di Orbassano, Torino, Italy 9

Hospital Fernando Fonseca, Amadora, Portugal 9

Hadassah University Hospital, Jerusalem, Israel 8

$\begin{array}{ll}\text { Universitatsspital Basel, Basel, Switzerland } & 7\end{array}$

Universita, Napoli, Italy $\quad 7$

University of Pécs, Pecs, Hungary $\quad 6$

Kaposi Mór Teaching Hospital, Kaposvar, Hungary $\quad 6$

University of Szeged, Szeged, Hungary $\quad 6$

G. Papanicolaou Hospital, Thessaloniki, Greece 5

UT Southwestern, Dallas, United States 4

Hospital San Bernanrdo, San Bernardo, Argentina 3

San Carlo Borromeo, Milan, Italy 3

Hospital sultanah Nora Ismail, Batu Pahat, Malaysia 3

Dr Lutfi Kirdar Kartal Education and Research Hosp., Istanbul, Turkey 3

Cheng Hsin General Hospital, Taipei, Taiwan, China 2

Hospiral Mancha Centro, Alcazar de San Juan, Spain 1

Sheba Medical Center, Ramat Gan, Israel

University of Maiduguri Teaching Hospital, Maiduguri, Nigeria 
$\%$ with

follow-up

in the

$\%$ MSBase previous

centre patients population year

001

002

003

004

005

006

007

008

009

010

011

012

013

014

015

016

017

018

019

020

021

022

023

024

025

026

027

028

029

030

031

032

033

034

035

036

037

038

039

040

041

042

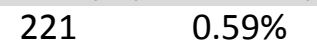

126

9

19

12

182

28

65

246

25

23

3

114

392

843

66

580

116

120

240

342

774

246

358

315

329

145

45

21

27

56

632

1

105

59

184

73

1308

16

864

1149
$0.34 \%$

$0.02 \%$

$0.05 \%$

$0.03 \%$

$0.49 \%$

$0.08 \%$

$0.17 \%$

$0.66 \%$

$0.07 \%$

$0.06 \%$

$0.01 \%$

$0.31 \%$

$1.05 \%$

$2.27 \%$

$0.18 \%$

$1.56 \%$

$0.31 \%$

$0.32 \%$

$0.65 \%$

$0.92 \%$

$2.08 \%$

$0.66 \%$

$0.96 \%$

$0.85 \%$

$0.88 \%$

$0.39 \%$

$0.12 \%$

$0.06 \%$

$0.07 \%$

$0.15 \%$

$1.70 \%$

$0.00 \%$

$0.28 \%$

$0.16 \%$

$0.49 \%$

$0.20 \%$

$3.52 \%$

$0.04 \%$

$2.32 \%$

$3.09 \%$

$0.02 \%$
$\%$ with first
cumulative EDSS within mean

follow-up the year of (years)

MS onset

$444 \quad 42 \%$

(years)

follow-up follow-up SD

2.0

2.6

1.3

567

$20 \%$

$56 \%$

$21 \%$

38

$0 \%$

$0 \%$

$5 \%$

0

647

33

217

953

88

93

$74 \%$

$100 \%$

$11 \%$

$27 \%$

$71 \%$

$0 \%$

$61 \%$

$1 \%$

$19 \%$

$33 \%$

$84 \%$

$67 \%$

$63 \%$

$74 \%$

$83 \%$

$2 \%$

$75 \%$

$91 \%$

$95 \%$

$63 \%$

$100 \%$

$53 \%$

$0 \%$

$0 \%$

$93 \%$

$97 \%$

$44 \%$

$59 \%$

$0 \%$
$67 \%$

$0 \%$

$18 \%$

$18 \%$

$28 \%$

$38 \%$

$24 \%$

$70 \%$

$0 \%$

$18 \%$

177

538

4668

$8 \%$

$23 \%$

$5 \%$

$18 \%$

$26 \%$

$15 \%$

$5 \%$

$9 \%$

$18 \%$

$22 \%$

$14 \%$

$18 \%$

$5 \%$

$15 \%$

$29 \%$

$10 \%$

$11 \%$

$29 \%$

$29 \%$

$100 \%$

$12 \%$

$68 \%$

$36 \%$

$59 \%$

$18 \%$

$6 \%$

$23 \%$

$33 \%$

0\%

$25 \%$

$57 \%$

11

15745

34
8087

6640
4.5

5.4

2.0

0.0

3.6

1.2

3.3

3.9

3.5

4.0

0.4

1.6

1.4

5.5

0.0

3.8

2.5

3.1

0.7

4.3

4.9

3.0

4.7

4.3

3.6

1.6

6.1

0.9

0.6

4.1

7.1

4.9

1.3

4.0

4.0

5.4

12.0

3.1

9.4

5.8

1.6
3.6

4.5

2.8

0.0

3.8

0.9

1.9

4.0

2.6

3.0

0.4

2.2 


\begin{tabular}{|c|c|c|c|c|c|c|c|c|}
\hline 043 & 161 & $0.43 \%$ & $0 \%$ & 1188 & $94 \%$ & 7.4 & 3.8 & 0.5 \\
\hline 044 & 3526 & $9.48 \%$ & $73 \%$ & 24042 & $30 \%$ & 6.8 & 4.9 & 0.7 \\
\hline 045 & 196 & $0.53 \%$ & $99 \%$ & 542 & $51 \%$ & 2.8 & 1.4 & 0.5 \\
\hline 046 & 151 & $0.41 \%$ & $0 \%$ & 3 & $1 \%$ & 0.0 & 0.1 & 5.0 \\
\hline 047 & 451 & $1.21 \%$ & $1 \%$ & 2379 & $37 \%$ & 5.3 & 5.5 & 1.0 \\
\hline 048 & 30 & $0.08 \%$ & $13 \%$ & 0 & $0 \%$ & 0.0 & 0.0 & 0.0 \\
\hline 049 & 300 & $0.81 \%$ & $69 \%$ & 1841 & $45 \%$ & 6.1 & 4.1 & 0.7 \\
\hline 050 & 135 & $0.36 \%$ & $87 \%$ & 924 & $39 \%$ & 6.8 & 4.6 & 0.7 \\
\hline 051 & 665 & $1.79 \%$ & $0 \%$ & 2835 & $25 \%$ & 4.3 & 4.6 & 1.1 \\
\hline 052 & 95 & $0.26 \%$ & $62 \%$ & 441 & $39 \%$ & 4.6 & 4.1 & 0.9 \\
\hline 053 & 1705 & $4.58 \%$ & $68 \%$ & 13096 & $33 \%$ & 7.7 & 5.9 & 0.8 \\
\hline 054 & 1 & $0.00 \%$ & $0 \%$ & 1 & $0 \%$ & 1.4 & 0.0 & 0.0 \\
\hline 055 & 190 & $0.51 \%$ & $89 \%$ & 704 & $37 \%$ & 3.7 & 2.6 & 0.7 \\
\hline 056 & 348 & $0.94 \%$ & $49 \%$ & 1894 & $17 \%$ & 5.4 & 4.5 & 0.8 \\
\hline 057 & 27 & $0.07 \%$ & $96 \%$ & 119 & $74 \%$ & 4.4 & 4.2 & 0.9 \\
\hline 058 & 176 & $0.47 \%$ & $0 \%$ & 131 & $88 \%$ & 0.7 & 1.3 & 1.7 \\
\hline 059 & 13 & $0.03 \%$ & $0 \%$ & 13 & $100 \%$ & 1.0 & 1.3 & 1.4 \\
\hline 060 & 207 & $0.56 \%$ & $0 \%$ & 528 & $16 \%$ & 2.5 & 3.6 & 1.4 \\
\hline 061 & 179 & $0.48 \%$ & $28 \%$ & 257 & $5 \%$ & 1.4 & 3.0 & 2.1 \\
\hline 062 & 96 & $0.26 \%$ & $82 \%$ & 190 & $25 \%$ & 2.0 & 2.0 & 1.0 \\
\hline 063 & 8 & $0.02 \%$ & $25 \%$ & 0 & $13 \%$ & 0.0 & 0.0 & 0.0 \\
\hline 064 & 15 & $0.04 \%$ & $0 \%$ & 0 & $7 \%$ & 0.0 & 0.0 & 0.0 \\
\hline 065 & 19 & $0.05 \%$ & $0 \%$ & 62 & $0 \%$ & 3.3 & 2.2 & 0.7 \\
\hline 066 & 78 & $0.21 \%$ & $0 \%$ & 332 & $18 \%$ & 4.3 & 3.6 & 0.9 \\
\hline 067 & 28 & $0.08 \%$ & $57 \%$ & 50 & $18 \%$ & 1.8 & 1.1 & 0.6 \\
\hline 068 & 24 & $0.06 \%$ & $88 \%$ & 174 & $38 \%$ & 7.3 & 4.0 & 0.6 \\
\hline 069 & 24 & $0.06 \%$ & $0 \%$ & 52 & $4 \%$ & 2.2 & 1.5 & 0.7 \\
\hline 070 & 19 & $0.05 \%$ & $0 \%$ & 26 & $0 \%$ & 1.4 & 0.9 & 0.6 \\
\hline 071 & 19 & $0.05 \%$ & $0 \%$ & 29 & $21 \%$ & 1.5 & 1.4 & 0.9 \\
\hline 072 & 13 & $0.03 \%$ & $0 \%$ & 28 & $0 \%$ & 2.2 & 1.1 & 0.5 \\
\hline 073 & 12 & $0.03 \%$ & $0 \%$ & 67 & $17 \%$ & 5.6 & 3.9 & 0.7 \\
\hline 074 & 6 & $0.02 \%$ & $0 \%$ & 21 & $17 \%$ & 3.5 & 2.7 & 0.8 \\
\hline 075 & 6 & $0.02 \%$ & $17 \%$ & 14 & $17 \%$ & 2.3 & 1.9 & 0.8 \\
\hline 076 & 6 & $0.02 \%$ & $83 \%$ & 39 & $83 \%$ & 6.4 & 4.7 & 0.7 \\
\hline 077 & 1 & $0.00 \%$ & $0 \%$ & 0 & $0 \%$ & 0.0 & 0.0 & 0.0 \\
\hline 078 & 8 & $0.02 \%$ & $0 \%$ & 39 & $0 \%$ & 4.9 & 4.5 & 0.9 \\
\hline 079 & 94 & $0.25 \%$ & $0 \%$ & 645 & $18 \%$ & 6.9 & 4.3 & 0.6 \\
\hline 080 & 599 & $1.61 \%$ & $11 \%$ & 564 & $27 \%$ & 1.0 & 2.5 & 2.6 \\
\hline 081 & 88 & $0.24 \%$ & $0 \%$ & 9 & $16 \%$ & 0.1 & 0.4 & 4.2 \\
\hline 082 & 1857 & $4.99 \%$ & $20 \%$ & 1824 & $10 \%$ & 1.0 & 1.3 & 1.4 \\
\hline 083 & 875 & $2.35 \%$ & $6 \%$ & 111 & $26 \%$ & 0.1 & 0.9 & 7.0 \\
\hline 084 & 9 & $0.02 \%$ & $0 \%$ & 3 & $67 \%$ & 0.3 & 0.3 & 0.8 \\
\hline 085 & 1872 & $5.03 \%$ & $38 \%$ & 12097 & $29 \%$ & 6.5 & 6.7 & 1.0 \\
\hline 086 & 528 & $1.42 \%$ & $52 \%$ & 3602 & $28 \%$ & 6.8 & 5.5 & 0.8 \\
\hline 087 & 1526 & $4.10 \%$ & $24 \%$ & 14109 & $40 \%$ & 9.2 & 5.6 & 0.6 \\
\hline 088 & 282 & $0.76 \%$ & $0 \%$ & 1281 & $22 \%$ & 4.5 & 4.2 & 0.9 \\
\hline 089 & 7 & $0.02 \%$ & $0 \%$ & 6 & $29 \%$ & 0.9 & 0.8 & 1.0 \\
\hline
\end{tabular}




\begin{tabular}{|c|c|c|c|c|c|c|c|c|}
\hline 090 & 429 & $1.15 \%$ & $0 \%$ & 4416 & $53 \%$ & 10.3 & 8.0 & 0.8 \\
\hline 091 & 621 & $1.67 \%$ & $36 \%$ & 2815 & $36 \%$ & 4.5 & 6.0 & 1.3 \\
\hline 092 & 291 & $0.78 \%$ & $60 \%$ & 1945 & $51 \%$ & 6.7 & 6.0 & 0.9 \\
\hline 093 & 360 & $0.97 \%$ & $64 \%$ & 1801 & $38 \%$ & 5.0 & 5.0 & 1.0 \\
\hline 094 & 3 & $0.01 \%$ & $67 \%$ & 1 & $33 \%$ & 0.4 & 0.4 & 0.9 \\
\hline 095 & 887 & $2.38 \%$ & $41 \%$ & 6692 & $47 \%$ & 7.6 & 7.1 & 0.9 \\
\hline 096 & 1200 & $3.23 \%$ & $55 \%$ & 1885 & $30 \%$ & 1.6 & 1.7 & 1.1 \\
\hline 097 & 12 & $0.03 \%$ & $0 \%$ & 7 & $75 \%$ & 0.6 & 0.7 & 1.2 \\
\hline 098 & 43 & $0.12 \%$ & $16 \%$ & 214 & $100 \%$ & 5.0 & 3.3 & 0.7 \\
\hline 099 & 106 & $0.28 \%$ & $1 \%$ & 293 & $37 \%$ & 2.8 & 3.2 & 1.2 \\
\hline 100 & 78 & $0.21 \%$ & $0 \%$ & 4 & $14 \%$ & 0.1 & 0.4 & 7.9 \\
\hline 101 & 22 & $0.06 \%$ & $45 \%$ & 17 & $50 \%$ & 0.8 & 1.6 & 2.1 \\
\hline 102 & 17 & $0.05 \%$ & $76 \%$ & 18 & $18 \%$ & 1.0 & 1.0 & 1.0 \\
\hline 103 & 3 & $0.01 \%$ & $67 \%$ & 0 & $100 \%$ & 0.1 & 0.1 & 0.9 \\
\hline 104 & 1 & $0.00 \%$ & $0 \%$ & 4 & $0 \%$ & 4.0 & 0.0 & 0.0 \\
\hline 105 & 1245 & $3.35 \%$ & $0 \%$ & 3368 & $11 \%$ & 2.7 & 3.8 & 1.4 \\
\hline 106 & 140 & $0.38 \%$ & $0 \%$ & 249 & $21 \%$ & 1.8 & 1.2 & 0.7 \\
\hline 107 & 748 & $2.01 \%$ & $24 \%$ & 6064 & $27 \%$ & 8.1 & 7.0 & 0.9 \\
\hline 108 & 402 & $1.08 \%$ & $0 \%$ & 248 & $1 \%$ & 0.6 & 1.2 & 1.9 \\
\hline 109 & 375 & $1.01 \%$ & $29 \%$ & 1532 & $29 \%$ & 4.1 & 4.5 & 1.1 \\
\hline 110 & 337 & $0.91 \%$ & $47 \%$ & 256 & $3 \%$ & 0.8 & 2.4 & 3.2 \\
\hline 111 & 292 & $0.79 \%$ & $0 \%$ & 2216 & $22 \%$ & 7.6 & 5.5 & 0.7 \\
\hline 112 & 9 & $0.02 \%$ & $0 \%$ & 6 & $22 \%$ & 0.6 & 0.4 & 0.6 \\
\hline 113 & 26 & $0.07 \%$ & $8 \%$ & 158 & $38 \%$ & 6.1 & 6.6 & 1.1 \\
\hline 114 & 73 & $0.20 \%$ & $81 \%$ & 69 & $8 \%$ & 0.9 & 1.0 & 1.1 \\
\hline 115 & 3 & $0.01 \%$ & $0 \%$ & 0 & $0 \%$ & 0.1 & 0.1 & 1.7 \\
\hline 116 & 1021 & $2.74 \%$ & $49 \%$ & 3556 & $39 \%$ & 3.5 & 4.4 & 1.3 \\
\hline 117 & 1081 & $2.91 \%$ & $49 \%$ & 3347 & $27 \%$ & 3.1 & 3.6 & 1.2 \\
\hline 118 & 46 & $0.12 \%$ & $70 \%$ & 201 & $46 \%$ & 4.4 & 4.8 & 1.1 \\
\hline 119 & 2 & $0.01 \%$ & $0 \%$ & 0 & $0 \%$ & 0.0 & 0.0 & 0.0 \\
\hline 120 & 108 & $0.29 \%$ & $10 \%$ & 175 & $33 \%$ & 1.6 & 1.9 & 1.2 \\
\hline 121 & 4 & $0.01 \%$ & $0 \%$ & 0 & $25 \%$ & 0.0 & 0.0 & 0.0 \\
\hline 122 & 40 & $0.11 \%$ & $0 \%$ & 0 & $0 \%$ & 0.0 & 0.0 & 0.0 \\
\hline
\end{tabular}


mean visit visit visit mean visit visit count visit count \% visits visits with frequency frequency frequency count

\begin{tabular}{|c|c|c|c|c|c|c|c|c|}
\hline SD & CoV & & with EDSS & no EDSS & (per year) & SD & CoV & relapses \\
\hline 5.0 & 5.4 & 1.1 & $87 \%$ & 0.7 & 7.2 & 54.7 & 7.6 & 515 \\
\hline 6.8 & 5.5 & 0.8 & $69 \%$ & 2.9 & 1.9 & 4.0 & 2.2 & 111 \\
\hline 2.8 & 1.3 & 0.5 & $100 \%$ & 0.0 & 1.1 & 1.7 & 1.6 & 23 \\
\hline 2.0 & 1.1 & 0.6 & $57 \%$ & 1.5 & 40.9 & 167.1 & 4.1 & 9 \\
\hline 0.8 & 0.5 & 0.6 & $64 \%$ & 0.4 & 0.0 & 0.0 & 0.0 & 12 \\
\hline 6.6 & 7.5 & 1.1 & $90 \%$ & 0.6 & 6.2 & 23.1 & 3.7 & 544 \\
\hline 2.6 & 1.3 & 0.5 & $73 \%$ & 0.9 & 2.2 & 2.0 & 0.9 & 42 \\
\hline 4.0 & 1.5 & 0.4 & $96 \%$ & 0.2 & 1.7 & 2.0 & 1.2 & 55 \\
\hline 9.5 & 12.1 & 1.3 & $99 \%$ & 0.1 & 4.1 & 23.4 & 5.7 & 566 \\
\hline 3.1 & 1.3 & 0.4 & $96 \%$ & 0.1 & 1.3 & 0.9 & 0.7 & 68 \\
\hline 9.2 & 6.2 & 0.7 & $99 \%$ & 0.1 & 2.9 & 1.5 & 0.5 & 56 \\
\hline 2.3 & 1.2 & 0.5 & $100 \%$ & 0.0 & 3.2 & 2.8 & 0.9 & 4 \\
\hline 3.4 & 3.5 & 1.1 & $69 \%$ & 1.5 & 2.0 & 3.2 & 1.6 & 8 \\
\hline 2.3 & 3.2 & 1.4 & $19 \%$ & 9.9 & 3.0 & 36.9 & 12.2 & 1635 \\
\hline 8.5 & 5.8 & 0.7 & $68 \%$ & 3.8 & 1.8 & 1.5 & 0.8 & 2658 \\
\hline 0.5 & 0.6 & 1.2 & $20 \%$ & 2.1 & 0.3 & 1.2 & 3.9 & 123 \\
\hline 7.9 & 6.3 & 0.8 & $91 \%$ & 0.6 & 2.4 & 2.6 & 1.1 & 2353 \\
\hline 3.9 & 2.8 & 0.7 & $96 \%$ & 0.2 & 1.8 & 1.4 & 0.8 & 263 \\
\hline 2.9 & 3.2 & 1.1 & $17 \%$ & 13.9 & 0.8 & 1.2 & 1.5 & 348 \\
\hline 2.4 & 1.3 & 0.5 & $92 \%$ & 0.2 & 3.1 & 4.2 & 1.3 & 88 \\
\hline 3.9 & 4.5 & 1.2 & $25 \%$ & 11.7 & 1.2 & 3.5 & 3.1 & 904 \\
\hline 7.4 & 6.0 & 0.8 & $61 \%$ & 4.6 & 1.6 & 1.5 & 0.9 & 2972 \\
\hline 5.8 & 3.4 & 0.6 & $72 \%$ & 2.0 & 2.3 & 2.0 & 0.9 & 631 \\
\hline 5.9 & 4.4 & 0.7 & $58 \%$ & 4.2 & 1.6 & 2.1 & 1.3 & 979 \\
\hline 5.3 & 4.0 & 0.8 & $67 \%$ & 2.6 & 2.3 & 4.7 & 2.0 & 1078 \\
\hline 2.4 & 0.9 & 0.4 & $91 \%$ & 0.2 & 1.2 & 1.6 & 1.3 & 7 \\
\hline 2.4 & 1.7 & 0.7 & $32 \%$ & 5.1 & 1.3 & 1.3 & 1.0 & 61 \\
\hline 7.9 & 7.1 & 0.9 & $33 \%$ & 15.7 & 3.3 & 7.9 & 2.4 & 202 \\
\hline 2.6 & 1.5 & 0.6 & $40 \%$ & 3.8 & 2.7 & 1.9 & 0.7 & 18 \\
\hline 1.9 & 1.5 & 0.8 & $96 \%$ & 0.1 & 1.5 & 2.3 & 1.6 & 29 \\
\hline 2.9 & 2.0 & 0.7 & $35 \%$ & 5.3 & 0.7 & 1.0 & 1.4 & 160 \\
\hline 8.4 & 6.3 & 0.8 & $67 \%$ & 4.2 & 1.7 & 4.1 & 2.5 & 2487 \\
\hline 10.0 & 0.0 & 0.0 & $100 \%$ & 0.0 & 2.1 & 0.0 & 0.0 & 3 \\
\hline 1.9 & 1.9 & 1.0 & $26 \%$ & 5.0 & 3.8 & 9.3 & 2.4 & 0 \\
\hline 6.3 & 4.2 & 0.7 & $98 \%$ & 0.1 & 2.7 & 3.6 & 1.3 & 131 \\
\hline 7.4 & 4.2 & 0.6 & $81 \%$ & 1.3 & 2.0 & 0.9 & 0.4 & 154 \\
\hline 12.8 & 7.6 & 0.6 & $99 \%$ & 0.1 & 2.6 & 0.9 & 0.4 & 174 \\
\hline 13.6 & 7.5 & 0.5 & $74 \%$ & 4.8 & 1.3 & 0.9 & 0.7 & 6806 \\
\hline 5.8 & 3.3 & 0.6 & $53 \%$ & 5.1 & 2.9 & 2.5 & 0.9 & 49 \\
\hline 12.5 & 6.8 & 0.5 & $75 \%$ & 4.2 & 1.5 & 0.8 & 0.5 & 4522 \\
\hline 7.7 & 5.6 & 0.7 & $98 \%$ & 0.1 & 1.5 & 1.9 & 1.2 & 2395 \\
\hline 4.6 & 4.2 & 0.9 & $97 \%$ & 0.1 & 2.6 & 2.7 & 1.0 & 17 \\
\hline
\end{tabular}




\begin{tabular}{|c|c|c|c|c|c|c|c|c|}
\hline 12.4 & 7.0 & 0.6 & $99 \%$ & 0.2 & 2.1 & 2.2 & 1.0 & 542 \\
\hline 22.0 & 18.8 & 0.9 & $99 \%$ & 0.2 & 3.3 & 2.2 & 0.7 & 13750 \\
\hline 9.2 & 2.9 & 0.3 & $100 \%$ & 0.0 & 4.0 & 1.6 & 0.4 & 594 \\
\hline 1.0 & 0.3 & 0.3 & $99 \%$ & 0.0 & 0.9 & 5.3 & 6.1 & 21 \\
\hline 3.6 & 2.7 & 0.7 & $99 \%$ & 0.0 & 3.0 & 6.2 & 2.1 & 2490 \\
\hline 0.0 & 0.2 & 5.5 & $4 \%$ & 0.8 & 0.0 & 0.0 & 0.0 & 58 \\
\hline 13.9 & 8.6 & 0.6 & $87 \%$ & 2.1 & 2.8 & 1.8 & 0.6 & 980 \\
\hline 13.1 & 7.9 & 0.6 & $72 \%$ & 4.7 & 2.1 & 1.2 & 0.6 & 661 \\
\hline 5.5 & 5.2 & 1.0 & $48 \%$ & 5.9 & 2.0 & 7.4 & 3.8 & 2426 \\
\hline 4.0 & 2.8 & 0.7 & $96 \%$ & 0.2 & 1.2 & 1.8 & 1.6 & 210 \\
\hline 13.0 & 9.1 & 0.7 & $99 \%$ & 0.1 & 2.3 & 3.3 & 1.4 & 7619 \\
\hline 4.0 & 0.0 & 0.0 & $100 \%$ & 0.0 & 2.8 & 0.0 & 0.0 & 1 \\
\hline 13.1 & 10.1 & 0.8 & $94 \%$ & 0.7 & 5.0 & 4.9 & 1.0 & 527 \\
\hline 6.7 & 5.0 & 0.7 & $96 \%$ & 0.3 & 1.2 & 1.2 & 1.0 & 1363 \\
\hline 8.7 & 6.8 & 0.8 & $100 \%$ & 0.0 & 3.0 & 2.9 & 1.0 & 97 \\
\hline 2.4 & 2.2 & 0.9 & $90 \%$ & 0.3 & 2.6 & 5.2 & 2.0 & 319 \\
\hline 2.2 & 1.3 & 0.6 & $78 \%$ & 0.6 & 2.0 & 2.3 & 1.2 & 12 \\
\hline 3.1 & 2.0 & 0.6 & $41 \%$ & 4.5 & 1.6 & 1.2 & 0.7 & 485 \\
\hline 1.7 & 1.9 & 1.1 & $39 \%$ & 2.6 & 0.4 & 0.8 & 1.8 & 774 \\
\hline 3.5 & 2.8 & 0.8 & $61 \%$ & 2.3 & 1.8 & 1.9 & 1.1 & 345 \\
\hline 0.3 & 0.5 & 1.9 & $29 \%$ & 0.6 & 0.0 & 0.0 & 0.0 & 24 \\
\hline 0.1 & 0.3 & 3.0 & $100 \%$ & 0.0 & 0.0 & 0.0 & 0.0 & 17 \\
\hline 28.6 & 17.8 & 0.6 & $95 \%$ & 1.5 & 9.0 & 3.3 & 0.4 & 136 \\
\hline 21.2 & 11.9 & 0.6 & $92 \%$ & 1.7 & 7.8 & 4.7 & 0.6 & 564 \\
\hline 21.0 & 12.7 & 0.6 & $98 \%$ & 0.3 & 12.2 & 2.3 & 0.2 & 111 \\
\hline 47.9 & 19.2 & 0.4 & $97 \%$ & 1.3 & 7.7 & 3.7 & 0.5 & 161 \\
\hline 25.3 & 19.0 & 0.8 & $96 \%$ & 1.2 & 12.7 & 5.3 & 0.4 & 132 \\
\hline 12.9 & 6.5 & 0.5 & $76 \%$ & 4.1 & 10.3 & 4.4 & 0.4 & 103 \\
\hline 9.7 & 10.3 & 1.1 & $72 \%$ & 3.8 & 7.8 & 5.4 & 0.7 & 84 \\
\hline 21.2 & 12.8 & 0.6 & $79 \%$ & 5.7 & 9.9 & 2.8 & 0.3 & 83 \\
\hline 18.3 & 6.5 & 0.4 & $71 \%$ & 7.4 & 4.6 & 2.8 & 0.6 & 86 \\
\hline 18.3 & 7.0 & 0.4 & $71 \%$ & 7.3 & 7.8 & 4.2 & 0.5 & 38 \\
\hline 17.3 & 14.1 & 0.8 & $50 \%$ & 17.5 & 5.3 & 4.8 & 0.9 & 62 \\
\hline 21.3 & 6.4 & 0.3 & $100 \%$ & 0.0 & 5.6 & 4.7 & 0.8 & 35 \\
\hline 1.0 & 0.0 & 0.0 & $100 \%$ & 0.0 & 0.0 & 0.0 & 0.0 & 0 \\
\hline 4.8 & 2.8 & 0.6 & $93 \%$ & 0.4 & 1.2 & 0.9 & 0.7 & 39 \\
\hline 23.7 & 15.4 & 0.7 & $98 \%$ & 0.5 & 3.6 & 1.2 & 0.3 & 444 \\
\hline 1.3 & 1.8 & 1.4 & $49 \%$ & 1.3 & 0.8 & 3.3 & 4.3 & 1334 \\
\hline 1.2 & 1.4 & 1.1 & $83 \%$ & 0.3 & 1.0 & 4.2 & 4.0 & 119 \\
\hline 2.7 & 3.3 & 1.2 & $98 \%$ & 0.0 & 2.3 & 9.4 & 4.1 & 445 \\
\hline 1.0 & 0.3 & 0.3 & $99 \%$ & 0.0 & 0.7 & 14.8 & 20.9 & 0 \\
\hline 2.8 & 1.5 & 0.5 & $96 \%$ & 0.1 & 17.8 & 28.6 & 1.6 & 16 \\
\hline 10.8 & 11.2 & 1.0 & $90 \%$ & 1.2 & 2.2 & 4.6 & 2.1 & 6184 \\
\hline 15.9 & 14.2 & 0.9 & $72 \%$ & 4.6 & 2.7 & 4.2 & 1.6 & 2435 \\
\hline 18.1 & 11.3 & 0.6 & $97 \%$ & 0.5 & 2.1 & 1.1 & 0.5 & 8715 \\
\hline 5.8 & 4.8 & 0.8 & $87 \%$ & 0.9 & 2.1 & 6.5 & 3.1 & 936 \\
\hline 3.4 & 2.4 & 0.7 & $83 \%$ & 0.7 & 3.2 & 2.6 & 0.8 & 8 \\
\hline
\end{tabular}




\begin{tabular}{|c|c|c|c|c|c|c|c|c|}
\hline 16.4 & 14.1 & 0.9 & $98 \%$ & 0.4 & 1.8 & 1.5 & 0.8 & 2167 \\
\hline 8.5 & 10.6 & 1.3 & $97 \%$ & 0.2 & 3.7 & 13.5 & 3.6 & 1836 \\
\hline 18.6 & 14.8 & 0.8 & $97 \%$ & 0.5 & 3.5 & 2.8 & 0.8 & 1137 \\
\hline 9.2 & 7.8 & 0.8 & $97 \%$ & 0.1 & 2.3 & 2.2 & 1.0 & 637 \\
\hline 2.3 & 1.5 & 0.7 & $100 \%$ & 0.0 & 3.3 & 3.8 & 1.1 & 4 \\
\hline 9.7 & 6.7 & 0.7 & $90 \%$ & 1.1 & 2.0 & 2.8 & 1.4 & 2508 \\
\hline 4.9 & 4.5 & 0.9 & $100 \%$ & 0.0 & 2.6 & 3.7 & 1.4 & 1222 \\
\hline 3.4 & 2.8 & 0.8 & $100 \%$ & 0.0 & 6.7 & 13.5 & 2.0 & 9 \\
\hline 10.6 & 6.6 & 0.6 & $94 \%$ & 0.6 & 6.2 & 21.9 & 3.6 & 76 \\
\hline 3.3 & 3.9 & 1.2 & $52 \%$ & 2.8 & 1.3 & 1.6 & 1.3 & 226 \\
\hline 1.0 & 0.3 & 0.2 & $96 \%$ & 0.0 & 0.1 & 0.5 & 7.5 & 20 \\
\hline 1.7 & 1.7 & 1.0 & $68 \%$ & 0.8 & 2.4 & 3.9 & 1.6 & 36 \\
\hline 2.1 & 0.8 & 0.4 & $100 \%$ & 0.0 & 2.6 & 4.0 & 1.5 & 42 \\
\hline 1.7 & 0.6 & 0.3 & $100 \%$ & 0.0 & 8.1 & 7.1 & 0.9 & 1 \\
\hline 17.0 & 0.0 & 0.0 & $100 \%$ & 0.0 & 4.3 & 0.0 & 0.0 & 9 \\
\hline 2.8 & 3.0 & 1.1 & $95 \%$ & 0.1 & 0.8 & 2.0 & 2.5 & 3230 \\
\hline 4.4 & 2.5 & 0.6 & $75 \%$ & 1.5 & 2.3 & 1.5 & 0.6 & 328 \\
\hline 10.5 & 7.0 & 0.7 & $72 \%$ & 3.6 & 1.8 & 2.2 & 1.2 & 2834 \\
\hline 1.2 & 1.3 & 1.1 & $98 \%$ & 0.0 & 0.8 & 1.7 & 2.1 & 231 \\
\hline 4.4 & 4.4 & 1.0 & $54 \%$ & 2.8 & 1.1 & 1.7 & 1.5 & 1070 \\
\hline 0.6 & 0.9 & 1.4 & $6 \%$ & 7.3 & 0.2 & 1.3 & 7.5 & 787 \\
\hline 13.6 & 8.7 & 0.6 & $78 \%$ & 3.9 & 2.0 & 1.3 & 0.7 & 512 \\
\hline 2.2 & 0.7 & 0.3 & $43 \%$ & 3.0 & 3.6 & 2.7 & 0.8 & 27 \\
\hline 5.0 & 2.5 & 0.5 & $97 \%$ & 0.1 & 1.2 & 0.7 & 0.6 & 53 \\
\hline 3.0 & 2.4 & 0.8 & $88 \%$ & 0.4 & 2.9 & 2.4 & 0.8 & 63 \\
\hline 1.3 & 0.6 & 0.4 & $57 \%$ & 1.0 & 3.9 & 6.8 & 1.7 & 3 \\
\hline 6.3 & 6.7 & 1.1 & $90 \%$ & 0.6 & 3.9 & 12.1 & 3.1 & 3116 \\
\hline 5.1 & 4.9 & 1.0 & $89 \%$ & 0.6 & 3.3 & 9.7 & 3.0 & 3805 \\
\hline 7.7 & 7.8 & 1.0 & $95 \%$ & 0.4 & 2.9 & 3.3 & 1.1 & 129 \\
\hline 0.0 & 0.0 & 0.0 & $0 \%$ & 0.5 & 0.0 & 0.0 & 0.0 & 3 \\
\hline 2.3 & 1.7 & 0.7 & $96 \%$ & 0.1 & 0.8 & 0.8 & 1.1 & 133 \\
\hline 1.0 & 0.0 & 0.0 & $100 \%$ & 0.0 & 0.0 & 0.0 & 0.0 & 4 \\
\hline 0.0 & 0.0 & 0.0 & $0 \%$ & 0.0 & 0.0 & 0.0 & 0.0 & 0 \\
\hline
\end{tabular}




\begin{tabular}{|c|c|c|c|c|c|c|}
\hline $\begin{array}{l}\text { mean } \\
\text { annualised } \\
\text { relapse rate }\end{array}$ & $\begin{array}{l}\text { annualised } \\
\text { relapse rate } \\
\text { SD }\end{array}$ & $\begin{array}{l}\text { annualised } \\
\text { relapse rate } \\
\text { CoV }\end{array}$ & $\begin{array}{l}\% \text { relapses } \\
\text { with clinical } \\
\text { information }\end{array}$ & $\begin{array}{l}\% \text { relapses } \\
\text { with } \\
\text { information } \\
\text { on duration }\end{array}$ & $\begin{array}{l}\% \text { relapses } \\
\text { with } \\
\text { information } \\
\text { on recovery }\end{array}$ & $\begin{array}{l}\text { \% relapses } \\
\text { with } \\
\text { information } \\
\text { on severity }\end{array}$ \\
\hline 1.2 & 34.8 & 30.0 & $97 \%$ & $50 \%$ & $59 \%$ & $74 \%$ \\
\hline 0.2 & 0.8 & 4.1 & $99 \%$ & $77 \%$ & $89 \%$ & $52 \%$ \\
\hline 0.5 & 4.5 & 9.5 & $100 \%$ & $96 \%$ & $100 \%$ & $100 \%$ \\
\hline 0.2 & 83.6 & 351.6 & $100 \%$ & $33 \%$ & $56 \%$ & $100 \%$ \\
\hline 0.0 & 0.0 & 0.0 & $100 \%$ & $83 \%$ & $100 \%$ & $100 \%$ \\
\hline 0.8 & 30.8 & 36.6 & $94 \%$ & $36 \%$ & $83 \%$ & $29 \%$ \\
\hline 1.3 & 3.0 & 2.4 & $100 \%$ & $86 \%$ & $100 \%$ & $100 \%$ \\
\hline 0.3 & 0.7 & 2.7 & $100 \%$ & $44 \%$ & $76 \%$ & $100 \%$ \\
\hline 0.6 & 12.1 & 20.3 & $97 \%$ & $52 \%$ & $76 \%$ & $15 \%$ \\
\hline 0.8 & 1.1 & 1.4 & $100 \%$ & $74 \%$ & $97 \%$ & $100 \%$ \\
\hline 0.6 & 0.6 & 0.9 & $91 \%$ & $89 \%$ & $98 \%$ & $95 \%$ \\
\hline 3.2 & 0.9 & 0.3 & $50 \%$ & $100 \%$ & $100 \%$ & $75 \%$ \\
\hline 0.0 & 0.1 & 1.4 & $50 \%$ & $63 \%$ & $38 \%$ & $38 \%$ \\
\hline 3.0 & 19.4 & 6.4 & $96 \%$ & $88 \%$ & $92 \%$ & $92 \%$ \\
\hline 0.6 & 1.7 & 2.9 & $85 \%$ & $76 \%$ & $67 \%$ & $69 \%$ \\
\hline 42.1 & 2.0 & 0.0 & $58 \%$ & $22 \%$ & $40 \%$ & $49 \%$ \\
\hline 1.1 & 3.2 & 3.0 & $94 \%$ & $68 \%$ & $81 \%$ & $78 \%$ \\
\hline 0.9 & 1.5 & 1.6 & $100 \%$ & $86 \%$ & $97 \%$ & $99 \%$ \\
\hline 1.0 & 2.3 & 2.3 & $71 \%$ & $12 \%$ & $43 \%$ & $49 \%$ \\
\hline 0.5 & 2.1 & 3.9 & $30 \%$ & $86 \%$ & $25 \%$ & $33 \%$ \\
\hline 0.6 & 5.1 & 8.4 & $1 \%$ & $2 \%$ & $5 \%$ & $5 \%$ \\
\hline 0.8 & 1.8 & 2.3 & $90 \%$ & $81 \%$ & $47 \%$ & $54 \%$ \\
\hline 0.9 & 2.3 & 2.7 & $77 \%$ & $37 \%$ & $37 \%$ & $36 \%$ \\
\hline 0.6 & 1.1 & 2.0 & $64 \%$ & $37 \%$ & $19 \%$ & $28 \%$ \\
\hline 0.8 & 6.3 & 7.9 & $91 \%$ & $84 \%$ & $27 \%$ & $21 \%$ \\
\hline 0.0 & 0.1 & 15.3 & $57 \%$ & $43 \%$ & $43 \%$ & $57 \%$ \\
\hline 0.3 & 1.0 & 3.8 & $79 \%$ & $15 \%$ & $67 \%$ & $90 \%$ \\
\hline 0.7 & 6.0 & 8.2 & $96 \%$ & $0 \%$ & $53 \%$ & $20 \%$ \\
\hline 1.0 & 1.4 & 1.4 & $100 \%$ & $94 \%$ & $94 \%$ & $50 \%$ \\
\hline 1.7 & 1.1 & 0.6 & $100 \%$ & $41 \%$ & $45 \%$ & $72 \%$ \\
\hline 0.7 & 0.7 & 1.0 & $88 \%$ & $84 \%$ & $30 \%$ & $20 \%$ \\
\hline 0.6 & 4.7 & 8.6 & $47 \%$ & $46 \%$ & $31 \%$ & $21 \%$ \\
\hline 0.6 & 0.0 & 0.0 & $100 \%$ & $67 \%$ & $100 \%$ & $67 \%$ \\
\hline 0.0 & 0.0 & 0.0 & $0 \%$ & $0 \%$ & $0 \%$ & $0 \%$ \\
\hline 0.6 & 1.5 & 2.6 & $94 \%$ & $98 \%$ & $97 \%$ & $92 \%$ \\
\hline 0.2 & 0.6 & 3.1 & $79 \%$ & $27 \%$ & $64 \%$ & $68 \%$ \\
\hline 0.4 & 0.6 & 1.4 & $96 \%$ & $26 \%$ & $88 \%$ & $96 \%$ \\
\hline 0.4 & 0.7 & 1.7 & $84 \%$ & $44 \%$ & $29 \%$ & $24 \%$ \\
\hline 1.4 & 4.8 & 3.3 & $100 \%$ & $22 \%$ & $33 \%$ & $100 \%$ \\
\hline 0.6 & 1.0 & 1.8 & $85 \%$ & $86 \%$ & $35 \%$ & $14 \%$ \\
\hline 0.4 & 1.5 & 4.1 & $77 \%$ & $7 \%$ & $59 \%$ & $53 \%$ \\
\hline 1.5 & 1.0 & 0.6 & $94 \%$ & $0 \%$ & $0 \%$ & $0 \%$ \\
\hline
\end{tabular}




\begin{tabular}{|c|c|c|c|c|c|c|}
\hline 0.5 & 1.8 & 3.9 & $99 \%$ & $98 \%$ & $98 \%$ & $99 \%$ \\
\hline 0.6 & 1.3 & 2.3 & $96 \%$ & $5 \%$ & $10 \%$ & $72 \%$ \\
\hline 1.1 & 0.9 & 0.9 & $100 \%$ & $0 \%$ & $2 \%$ & $100 \%$ \\
\hline 6.1 & 0.8 & 0.1 & $100 \%$ & $86 \%$ & $100 \%$ & $100 \%$ \\
\hline 1.0 & 7.8 & 7.5 & $92 \%$ & $97 \%$ & $54 \%$ & $54 \%$ \\
\hline 0.0 & 0.0 & 0.0 & $86 \%$ & $53 \%$ & $84 \%$ & $84 \%$ \\
\hline 0.5 & 1.3 & 2.5 & $79 \%$ & $71 \%$ & $48 \%$ & $70 \%$ \\
\hline 0.7 & 0.6 & 0.9 & $62 \%$ & $8 \%$ & $11 \%$ & $8 \%$ \\
\hline 0.9 & 17.9 & 20.9 & $46 \%$ & $7 \%$ & $10 \%$ & $11 \%$ \\
\hline 0.5 & 1.7 & 3.7 & $88 \%$ & $11 \%$ & $17 \%$ & $10 \%$ \\
\hline 0.6 & 4.5 & 7.8 & $69 \%$ & $12 \%$ & $11 \%$ & $4 \%$ \\
\hline 0.7 & 0.0 & 0.0 & $0 \%$ & $0 \%$ & $0 \%$ & $0 \%$ \\
\hline 0.7 & 4.6 & 6.1 & $96 \%$ & $96 \%$ & $94 \%$ & $95 \%$ \\
\hline 0.7 & 2.2 & 3.1 & $77 \%$ & $12 \%$ & $79 \%$ & $73 \%$ \\
\hline 0.8 & 3.4 & 4.1 & $99 \%$ & $100 \%$ & $100 \%$ & $100 \%$ \\
\hline 2.4 & 5.6 & 2.3 & $100 \%$ & $20 \%$ & $5 \%$ & $7 \%$ \\
\hline 0.9 & 2.3 & 2.5 & $100 \%$ & $50 \%$ & $58 \%$ & $75 \%$ \\
\hline 0.9 & 1.8 & 2.0 & $96 \%$ & $25 \%$ & $41 \%$ & $27 \%$ \\
\hline 3.0 & 1.7 & 0.6 & $66 \%$ & $18 \%$ & $5 \%$ & $0 \%$ \\
\hline 1.8 & 2.5 & 1.4 & $78 \%$ & $52 \%$ & $68 \%$ & $58 \%$ \\
\hline 0.0 & 0.0 & 0.0 & $58 \%$ & $33 \%$ & $4 \%$ & $4 \%$ \\
\hline 0.0 & 0.0 & 0.0 & $100 \%$ & $94 \%$ & $24 \%$ & $24 \%$ \\
\hline 2.2 & 2.3 & 1.0 & $50 \%$ & $0 \%$ & $7 \%$ & $24 \%$ \\
\hline 1.7 & 5.8 & 3.4 & $76 \%$ & $10 \%$ & $40 \%$ & $40 \%$ \\
\hline 2.2 & 3.4 & 1.5 & $95 \%$ & $73 \%$ & $86 \%$ & $92 \%$ \\
\hline 0.9 & 0.9 & 1.0 & $98 \%$ & $88 \%$ & $93 \%$ & $91 \%$ \\
\hline 2.5 & 14.1 & 5.6 & $86 \%$ & $1 \%$ & $17 \%$ & $8 \%$ \\
\hline 3.9 & 5.2 & 1.3 & $82 \%$ & $0 \%$ & $40 \%$ & $41 \%$ \\
\hline 2.9 & 9.9 & 3.5 & $60 \%$ & $0 \%$ & $1 \%$ & $12 \%$ \\
\hline 2.9 & 2.4 & 0.8 & $12 \%$ & $37 \%$ & $1 \%$ & $5 \%$ \\
\hline 1.3 & 5.1 & 3.9 & $55 \%$ & $3 \%$ & $0 \%$ & $1 \%$ \\
\hline 1.8 & 1.8 & 1.0 & $87 \%$ & $0 \%$ & $0 \%$ & $0 \%$ \\
\hline 4.5 & 2.5 & 0.5 & $68 \%$ & $3 \%$ & $5 \%$ & $5 \%$ \\
\hline 0.9 & 1.2 & 1.3 & $100 \%$ & $63 \%$ & $97 \%$ & $97 \%$ \\
\hline 0.0 & 0.0 & 0.0 & $0 \%$ & $0 \%$ & $0 \%$ & $0 \%$ \\
\hline 1.0 & 2.3 & 2.3 & $100 \%$ & $51 \%$ & $0 \%$ & $100 \%$ \\
\hline 0.7 & 0.7 & 1.0 & $100 \%$ & $79 \%$ & $48 \%$ & $100 \%$ \\
\hline 2.4 & 3.9 & 1.7 & $57 \%$ & $18 \%$ & $36 \%$ & $33 \%$ \\
\hline 12.9 & 3.7 & 0.3 & $37 \%$ & $50 \%$ & $43 \%$ & $38 \%$ \\
\hline 0.2 & 3.8 & 15.5 & $95 \%$ & $2 \%$ & $12 \%$ & $12 \%$ \\
\hline 0.0 & 0.0 & 0.0 & $0 \%$ & $0 \%$ & $0 \%$ & $0 \%$ \\
\hline 5.1 & 44.4 & 8.6 & $100 \%$ & $100 \%$ & $100 \%$ & $100 \%$ \\
\hline 0.5 & 3.2 & 6.2 & $78 \%$ & $4 \%$ & $54 \%$ & $40 \%$ \\
\hline 0.7 & 3.2 & 4.8 & $78 \%$ & $14 \%$ & $28 \%$ & $24 \%$ \\
\hline 0.6 & 0.9 & 1.5 & $91 \%$ & $14 \%$ & $17 \%$ & $17 \%$ \\
\hline 0.7 & 4.9 & 6.7 & $100 \%$ & $9 \%$ & $57 \%$ & $7 \%$ \\
\hline 1.3 & 1.1 & 0.8 & $100 \%$ & $25 \%$ & $75 \%$ & $13 \%$ \\
\hline
\end{tabular}




\begin{tabular}{|c|c|c|c|c|c|c|}
\hline 0.5 & 1.0 & 2.1 & $81 \%$ & $10 \%$ & $13 \%$ & $9 \%$ \\
\hline 0.7 & 9.6 & 14.7 & $82 \%$ & $29 \%$ & $31 \%$ & $17 \%$ \\
\hline 0.6 & 2.5 & 4.3 & $93 \%$ & $40 \%$ & $40 \%$ & $52 \%$ \\
\hline 0.4 & 1.5 & 4.1 & $98 \%$ & $94 \%$ & $96 \%$ & $95 \%$ \\
\hline 3.0 & 2.2 & 0.7 & $100 \%$ & $100 \%$ & $100 \%$ & $100 \%$ \\
\hline 0.4 & 1.8 & 4.7 & $92 \%$ & $15 \%$ & $45 \%$ & $20 \%$ \\
\hline 0.6 & 2.3 & 3.6 & $94 \%$ & $85 \%$ & $82 \%$ & $82 \%$ \\
\hline 1.3 & 0.7 & 0.5 & $100 \%$ & $100 \%$ & $100 \%$ & $100 \%$ \\
\hline 0.4 & 11.1 & 31.1 & $74 \%$ & $37 \%$ & $84 \%$ & $75 \%$ \\
\hline 0.8 & 1.6 & 2.0 & $81 \%$ & $15 \%$ & $43 \%$ & $23 \%$ \\
\hline 4.7 & 0.2 & 0.0 & $100 \%$ & $40 \%$ & $85 \%$ & $65 \%$ \\
\hline 2.1 & 2.7 & 1.3 & $86 \%$ & $42 \%$ & $42 \%$ & $47 \%$ \\
\hline 2.4 & 2.1 & 0.9 & $100 \%$ & $12 \%$ & $40 \%$ & $36 \%$ \\
\hline 3.0 & 0.0 & 0.0 & $100 \%$ & $100 \%$ & $100 \%$ & $100 \%$ \\
\hline 2.3 & 0.0 & 0.0 & $100 \%$ & $11 \%$ & $33 \%$ & $100 \%$ \\
\hline 1.0 & 2.8 & 2.9 & $100 \%$ & $0 \%$ & $1 \%$ & $9 \%$ \\
\hline 1.3 & 1.6 & 1.2 & $100 \%$ & $32 \%$ & $43 \%$ & $26 \%$ \\
\hline 0.5 & 1.9 & 4.0 & $96 \%$ & $1 \%$ & $36 \%$ & $10 \%$ \\
\hline 0.9 & 0.7 & 0.7 & $0 \%$ & $2 \%$ & $1 \%$ & $0 \%$ \\
\hline 0.7 & 2.4 & 3.4 & $73 \%$ & $66 \%$ & $75 \%$ & $40 \%$ \\
\hline 3.1 & 2.4 & 0.8 & $36 \%$ & $17 \%$ & $26 \%$ & $23 \%$ \\
\hline 0.2 & 0.5 & 2.2 & $100 \%$ & $71 \%$ & $73 \%$ & $62 \%$ \\
\hline 4.7 & 7.6 & 1.6 & $100 \%$ & $41 \%$ & $7 \%$ & $100 \%$ \\
\hline 0.3 & 0.4 & 1.1 & $94 \%$ & $89 \%$ & $94 \%$ & $92 \%$ \\
\hline 0.9 & 2.0 & 2.2 & $87 \%$ & $49 \%$ & $68 \%$ & $60 \%$ \\
\hline 17.7 & 6.8 & 0.4 & $100 \%$ & $33 \%$ & $33 \%$ & $100 \%$ \\
\hline 0.9 & 15.4 & 17.6 & $93 \%$ & $60 \%$ & $79 \%$ & $72 \%$ \\
\hline 1.1 & 8.8 & 7.7 & $97 \%$ & $27 \%$ & $65 \%$ & $73 \%$ \\
\hline 0.6 & 3.4 & 5.3 & $94 \%$ & $67 \%$ & $76 \%$ & $70 \%$ \\
\hline 0.0 & 0.0 & 0.0 & $67 \%$ & $100 \%$ & $100 \%$ & $33 \%$ \\
\hline 0.8 & 0.6 & 0.7 & $68 \%$ & $38 \%$ & $53 \%$ & $20 \%$ \\
\hline 0.0 & 0.0 & 0.0 & $100 \%$ & $100 \%$ & $100 \%$ & $100 \%$ \\
\hline 0.0 & 0.0 & 0.0 & $0 \%$ & $0 \%$ & $0 \%$ & $0 \%$ \\
\hline
\end{tabular}


$\%$ relapses $\%$ relapses

with treatment information information (nr) $35 \%$ $66 \%$ $100 \%$ $100 \%$

$100 \%$

$53 \%$

$45 \%$

$80 \%$

$38 \%$

$51 \%$

$75 \%$

$100 \%$

$75 \%$

$35 \%$

$58 \%$

$53 \%$

$51 \%$

$47 \%$

$29 \%$

$40 \%$

$87 \%$

$45 \%$

$26 \%$

$17 \%$

$28 \%$

$43 \%$

$77 \%$

$64 \%$

$72 \%$

$55 \%$

$39 \%$

$47 \%$

$33 \%$

$0 \%$

$64 \%$

$40 \%$

$46 \%$

$7 \%$

$14 \%$

$35 \%$

$16 \%$

$53 \%$

with

$35 \%$

$100 \%$

$56 \%$

$100 \%$

$14 \%$

$100 \%$

$76 \%$

$6 \%$

$97 \%$

$59 \%$

$50 \%$

$38 \%$

$31 \%$

$26 \%$

$22 \%$

$35 \%$

$44 \%$

$10 \%$

$3 \%$

$0 \%$

$17 \%$

$15 \%$

$2 \%$

$4 \%$

$0 \%$

$43 \%$

$12 \%$

$33 \%$

$21 \%$

$9 \%$

$12 \%$

$33 \%$

$0 \%$

$55 \%$

$17 \%$

$36 \%$

$1 \%$

$33 \%$

$3 \%$

$12 \%$

$0 \%$ complete female $15 \% \quad 138 \quad 62 \% \quad 1003$

$11 \quad 58 \% \quad 25$

12

859

65

102

788

46

91

4

476

1781

3351

156

4278

322

829

1323

2229

2894

1252

3150

1452

213

474

313

100

67

221

1595

7

345

136

545

360

3620

23

$870 \quad 76 \%$

$$
\begin{array}{llll} 
& M R I & \text { MRI } & \text { MRI } \\
\text { MRI } & \text { frequency } & \text { frequency } & \text { frequency }
\end{array}
$$
(peryear) SD CoV

$\begin{array}{lll}2.3 & 54.8 & 24.2\end{array}$

$\begin{array}{lll}0.8 & 9.2 & 11.3\end{array}$

$\begin{array}{lll}0.7 & 8.1 & 11.0\end{array}$

$\begin{array}{lll}0.7 & 167.3 \quad 253.1\end{array}$
0.0

1.3

2.0

0.5

0.8

0.5

1.0

3.2

2.7

3.3

0.7

53.5

1.9

1.1

2.3

8.1

1.5

0.8

1.7

1.9

1.1

0.2

2.1

1.1

5.4

4.0

1.0

0.4

1.4

3.0

0.6

0.7

0.9

0.2

0.7
0.0

20.3

$13-0.6$

$1.9 \quad 4.0$

$46.6 \quad 56.4$

$1.0 \quad 1.8$

$0.8 \quad 0.8$

$\begin{array}{ll}2.0 & 0.6\end{array}$

$3.9 \quad 1.5$

$55.5 \quad 16.8$

$1.9 \quad 2.7$

$1.3 \quad 0.0$

$4.0 \quad 2.1$

$1.6 \quad 1.4$

$5.0 \quad 2.2$

$6.7 \quad 0.8$

$10.5 \quad 7.0$

$2.0 \quad 2.6$

$2.3 \quad 1.4$

$4.4 \quad 2.4$

$5.2 \quad 4.9$

$0.9 \quad 5.3$

$2.3 \quad 1.1$

$16.3 \quad 14.3$

$5.9 \quad 1.1$

$2.4 \quad 0.6$

$1.0 \quad 1.1$

$2.3 \quad 6.6$

$0.0 \quad 0.0$

$12.1 \quad 4.1$

$1.5 \quad 2.6$

$1.0 \quad 1.4$

$3.3 \quad 3.6$

$1.0 \quad 4.4$

$\begin{array}{ll}1.8 & 2.7\end{array}$
3668

3129

13

0.5

0.5

1.2

$86 \%$

1.7

3.8

$6 \quad 86 \% \quad 13$

2.0

4.2

$1.2 \quad 1.0$ 


\begin{tabular}{|c|c|c|c|c|c|c|c|}
\hline $67 \%$ & $66 \%$ & 119 & $74 \%$ & 746 & 0.6 & 0.9 & 1.5 \\
\hline $70 \%$ & $8 \%$ & 2514 & $71 \%$ & 24181 & 1.0 & 1.1 & 1.1 \\
\hline $47 \%$ & $2 \%$ & 135 & $69 \%$ & 760 & 1.4 & 1.2 & 0.9 \\
\hline $100 \%$ & $100 \%$ & 106 & $70 \%$ & 0 & 0.0 & 0.0 & 0.0 \\
\hline $25 \%$ & $12 \%$ & 300 & $67 \%$ & 192 & 0.1 & 1.8 & 22.6 \\
\hline $83 \%$ & $62 \%$ & 23 & $77 \%$ & 0 & 0.0 & 0.0 & 0.0 \\
\hline $52 \%$ & $19 \%$ & 208 & $69 \%$ & 1115 & 0.6 & 1.5 & 2.5 \\
\hline $48 \%$ & $2 \%$ & 84 & $62 \%$ & 937 & 1.0 & 1.1 & 1.1 \\
\hline $26 \%$ & $3 \%$ & 451 & $68 \%$ & 1441 & 0.5 & 17.9 & 35.2 \\
\hline $27 \%$ & $5 \%$ & 74 & $78 \%$ & 530 & 1.2 & 1.5 & 1.3 \\
\hline $32 \%$ & $1 \%$ & 1137 & $67 \%$ & 3830 & 0.3 & 1.6 & 5.5 \\
\hline $0 \%$ & $0 \%$ & 1 & $100 \%$ & 0 & 0.0 & 0.0 & 0.0 \\
\hline $47 \%$ & $41 \%$ & 124 & $65 \%$ & 1847 & 2.6 & 8.9 & 3.4 \\
\hline $57 \%$ & $28 \%$ & 242 & $70 \%$ & 1543 & 0.8 & 1.6 & 1.9 \\
\hline $69 \%$ & $69 \%$ & 21 & $78 \%$ & 128 & 1.1 & 2.6 & 2.5 \\
\hline $33 \%$ & $3 \%$ & 125 & $71 \%$ & 352 & 2.7 & 3.8 & 1.4 \\
\hline $67 \%$ & $50 \%$ & 8 & $62 \%$ & 19 & 1.5 & 1.9 & 1.3 \\
\hline $23 \%$ & $8 \%$ & 138 & $67 \%$ & 289 & 0.5 & 1.1 & 2.0 \\
\hline $9 \%$ & $0 \%$ & 130 & $73 \%$ & 396 & 1.5 & 1.3 & 0.9 \\
\hline $25 \%$ & $17 \%$ & 70 & $73 \%$ & 244 & 1.3 & 2.1 & 1.7 \\
\hline $13 \%$ & $0 \%$ & 5 & $63 \%$ & 58 & 0.0 & 0.0 & 0.0 \\
\hline $12 \%$ & $0 \%$ & 8 & $53 \%$ & 12 & 0.0 & 0.0 & 0.0 \\
\hline $65 \%$ & $4 \%$ & 15 & $79 \%$ & 75 & 1.2 & 1.4 & 1.1 \\
\hline $68 \%$ & $24 \%$ & 60 & $77 \%$ & 199 & 0.6 & 2.0 & 3.3 \\
\hline $75 \%$ & $60 \%$ & 16 & $57 \%$ & 81 & 1.6 & 2.0 & 1.3 \\
\hline $84 \%$ & $78 \%$ & 15 & $63 \%$ & 279 & 1.6 & 1.1 & 0.7 \\
\hline $83 \%$ & $3 \%$ & 18 & $75 \%$ & 125 & 2.4 & 10.4 & 4.4 \\
\hline $78 \%$ & $37 \%$ & 11 & $58 \%$ & 74 & 2.8 & 2.2 & 0.8 \\
\hline $80 \%$ & $1 \%$ & 16 & $84 \%$ & 69 & 2.4 & 8.5 & 3.6 \\
\hline $67 \%$ & $0 \%$ & 10 & $77 \%$ & 91 & 3.2 & 2.9 & 0.9 \\
\hline $65 \%$ & $0 \%$ & 11 & $92 \%$ & 56 & 0.8 & 2.5 & 3.0 \\
\hline $84 \%$ & $0 \%$ & 4 & $67 \%$ & 40 & 1.9 & 2.9 & 1.5 \\
\hline $71 \%$ & $5 \%$ & 5 & $83 \%$ & 35 & 2.5 & 1.7 & 0.7 \\
\hline $94 \%$ & $89 \%$ & 6 & $100 \%$ & 26 & 0.7 & 1.1 & 1.6 \\
\hline $0 \%$ & $0 \%$ & 0 & $0 \%$ & 0 & 0.0 & 0.0 & 0.0 \\
\hline $13 \%$ & $0 \%$ & 3 & $38 \%$ & 0 & 0.0 & 0.0 & 0.0 \\
\hline $50 \%$ & $48 \%$ & 63 & $67 \%$ & 203 & 0.3 & 1.1 & 3.5 \\
\hline $32 \%$ & $13 \%$ & 394 & $66 \%$ & 2233 & 4.0 & 6.2 & 1.6 \\
\hline $31 \%$ & $15 \%$ & 55 & $63 \%$ & 56 & 6.1 & 0.6 & 0.1 \\
\hline $13 \%$ & $10 \%$ & 1481 & $80 \%$ & 2 & 0.0 & 0.0 & 5.5 \\
\hline $0 \%$ & $0 \%$ & 658 & $75 \%$ & 0 & 0.0 & 0.0 & 0.0 \\
\hline $50 \%$ & $100 \%$ & 6 & $67 \%$ & 27 & 8.7 & 89.2 & 10.3 \\
\hline $74 \%$ & $28 \%$ & 1266 & $68 \%$ & 13099 & 1.1 & 4.0 & 3.7 \\
\hline $48 \%$ & $3 \%$ & 379 & $72 \%$ & 3924 & 1.1 & 3.4 & 3.1 \\
\hline $29 \%$ & $7 \%$ & 1027 & $67 \%$ & 22302 & 1.6 & 2.7 & 1.7 \\
\hline $54 \%$ & $4 \%$ & 194 & $69 \%$ & 1074 & 0.8 & 11.9 & 14.2 \\
\hline $25 \%$ & $13 \%$ & 4 & $57 \%$ & 15 & 2.5 & 2.8 & 1.1 \\
\hline
\end{tabular}




$\begin{array}{rrrrrrrr}13 \% & 5 \% & 289 & 67 \% & 1102 & 0.2 & 0.9 & 3.5 \\ 41 \% & 6 \% & 418 & 67 \% & 3514 & 1.2 & 19.5 & 15.6 \\ 62 \% & 12 \% & 193 & 66 \% & 3127 & 1.6 & 5.5 & 3.4 \\ 72 \% & 67 \% & 237 & 66 \% & 3101 & 1.7 & 3.0 & 1.7 \\ 50 \% & 50 \% & 2 & 67 \% & 6 & 4.5 & 1.9 & 0.4 \\ 59 \% & 10 \% & 611 & 69 \% & 6243 & 0.9 & 4.5 & 4.8 \\ 75 \% & 58 \% & 790 & 66 \% & 3120 & 1.7 & 2.8 & 1.7 \\ 89 \% & 100 \% & 5 & 42 \% & 7 & 1.0 & 7.0 & 7.1 \\ 50 \% & 30 \% & 27 & 63 \% & 77 & 0.4 & 0.5 & 1.5 \\ 64 \% & 11 \% & 63 & 59 \% & 427 & 1.5 & 3.5 & 2.4 \\ 50 \% & 65 \% & 56 & 72 \% & 13 & 3.1 & 0.1 & 0.0 \\ 22 \% & 8 \% & 17 & 77 \% & 47 & 2.8 & 3.5 & 1.3 \\ 19 \% & 14 \% & 15 & 88 \% & 2 & 0.1 & 0.0 & 0.0 \\ 100 \% & 100 \% & 2 & 67 \% & 5 & 15.2 & 7.1 & 0.5 \\ 100 \% & 33 \% & 1 & 100 \% & 2 & 0.5 & 0.0 & 0.0 \\ 55 \% & 1 \% & 853 & 69 \% & 924 & 0.3 & 1.5 & 5.6 \\ 91 \% & 23 \% & 109 & 78 \% & 126 & 0.5 & 0.6 & 1.3 \\ 50 \% & 1 \% & 538 & 72 \% & 2815 & 0.5 & 1.6 & 3.5 \\ 1 \% & 0 \% & 274 & 68 \% & 0 & 0.0 & 0.0 & 0.0 \\ 82 \% & 29 \% & 272 & 73 \% & 1273 & 0.8 & 2.3 & 2.8 \\ 83 \% & 10 \% & 242 & 72 \% & 314 & 1.2 & 0.2 & 0.2 \\ 81 \% & 43 \% & 206 & 71 \% & 1430 & 0.6 & 0.8 & 1.2 \\ 70 \% & 7 \% & 9 & 100 \% & 22 & 3.8 & 4.6 & 1.2 \\ 70 \% & 64 \% & 15 & 58 \% & 78 & 0.5 & 0.6 & 1.2 \\ 56 \% & 25 \% & 43 & 59 \% & 112 & 1.6 & 2.0 & 1.3 \\ 67 \% & 33 \% & 1 & 33 \% & 4 & 23.6 & 3.4 & 0.1 \\ 57 \% & 38 \% & 669 & 66 \% & 4252 & 1.2 & 11.5 & 9.6 \\ 49 \% & 34 \% & 741 & 69 \% & 4483 & 1.3 & 10.9 & 8.1 \\ 74 \% & 48 \% & 29 & 63 \% & 245 & 1.2 & 5.0 & 4.1 \\ 100 \% & 33 \% & 2 & 100 \% & 2 & 0.0 & 0.0 & 0.0 \\ 43 \% & 11 \% & 83 & 77 \% & 315 & 1.8 & 1.3 & 0.7 \\ 75 \% & 100 \% & 4 & 100 \% & 5 & 0.0 & 0.0 & 0.0 \\ 0 \% & 0 \% & 29 & 73 \% & 28 & 0.0 & 0.0 & 0.0\end{array}$


$\%$ MRI with $\quad \%$ MRI with

$\% \mathrm{MRI}$ with information on information enhancing on $\mathrm{T} 2$ lesions lesions

\section{$95 \%$}

$98 \%$

$94 \%$

$96 \%$

$25 \%$

$98 \%$

$94 \%$

$88 \%$

$99 \%$

$96 \%$

$91 \%$

$100 \%$

$0 \%$

$60 \%$

$51 \%$

$7 \%$

$96 \%$

$80 \%$

$24 \%$

$1 \%$

$0 \%$

$47 \%$

$55 \%$

$1 \%$

95\%

2\%

$1 \%$

$13 \%$

$87 \%$

$43 \%$

$91 \%$

$38 \%$

$100 \%$

$5 \%$

$71 \%$

$5 \%$

99\%

$98 \%$

$4 \%$

$59 \%$

$92 \%$

$0 \%$

on T2 lesion

$100 \%$

$96 \%$

$100 \%$

$90 \%$

$34 \%$

$76 \%$

$95 \%$

$70 \%$

$91 \%$

$100 \%$

$0 \%$

$35 \%$

$21 \%$

$6 \%$

$38 \%$

$54 \%$

$1 \%$

$0 \%$

$0 \%$

$5 \%$

$47 \%$

$2 \%$

$1 \%$

$0 \%$

$3 \%$

$1 \%$

$77 \%$

$15 \%$

$0 \%$

$35 \%$

$100 \%$

$100 \%$

$93 \%$

$5 \%$

$4 \%$

$12 \%$

$9 \%$

$39 \%$

$13 \%$

$0 \%$ information count information CSF count information results

$\begin{array}{rrrrrr}93 \% & 1 \% & 1 \% & 107 & 48 \% & 85 \% \\ 96 \% & 6 \% & 6 \% & 73 & 58 \% & 96 \%\end{array}$

$100 \%$

$24 \%$

$100 \%$

$2 \%$

$26 \%$

$42 \%$

$11 \%$

$0 \%$

$8 \%$

$25 \%$

$0 \%$

$23 \%$

$24 \%$

$7 \%$

$55 \%$

$53 \%$

$13 \%$

$1 \%$

$0 \%$

$31 \%$

$65 \%$

$3 \%$

$26 \%$

$0 \%$

$4 \%$

$9 \%$

$64 \%$

$34 \%$

$38 \%$

$22 \%$

$71 \%$

$100 \%$

$54 \%$

$5 \%$

$81 \%$

$11 \%$

$9 \%$

$28 \%$

$35 \%$

$0 \%$

\section{$94 \%$}

$24 \%$

$25 \%$

$2 \%$

$20 \%$

$35 \%$

$11 \%$

$0 \%$

$8 \%$

$25 \%$

$0 \%$

$16 \%$

$6 \%$

$0 \%$

$29 \%$

$42 \%$

$1 \%$

$0 \%$

$0 \%$

$1 \%$

$22 \%$

$1 \%$

$0 \%$

$0 \%$

$0 \%$

$0 \%$

$43 \%$

$3 \%$

$0 \%$

$15 \%$

$71 \%$

$5 \%$

$41 \%$

$1 \%$

$3 \%$

$1 \%$

$4 \%$

$10 \%$

$5 \%$

$0 \%$
$58 \%$

$100 \%$

$100 \%$

$100 \% \quad 42 \%$

$40 \% \quad 92 \%$

$14 \% \quad 100 \%$

$17 \% \quad 82 \%$

$49 \% \quad 93 \%$

$20 \% \quad 100 \%$

$57 \% \quad 92 \%$

$0 \% \quad 0 \%$

$1 \% \quad 100 \%$

$15 \% \quad 91 \%$

$20 \% \quad 73 \%$

$15 \% \quad 90 \%$

$64 \% \quad 73 \%$

$24 \% \quad 100 \%$

$24 \% \quad 55 \%$

$2 \% \quad 0 \%$

$15 \% \quad 4 \%$

$13 \% \quad 50 \%$

$20 \% \quad 67 \%$

$35 \% \quad 87 \%$

$16 \% \quad 12 \%$

$0 \% \quad 0 \%$

$12 \% \quad 78 \%$

$42 \% \quad 68 \%$

$14 \% \quad 100 \%$

$15 \% \quad 100 \%$

$38 \% \quad 0 \%$

$51 \% \quad 30 \%$

$0 \% \quad 0 \%$

$51 \% \quad 69 \%$

$41 \% \quad 100 \%$

$6 \% \quad 36 \%$

$33 \% \quad 79 \%$

$33 \% \quad 97 \%$

$25 \% \quad 50 \%$

$46 \% \quad 54 \%$

$22 \% \quad 98 \%$

$71 \% \quad 0 \%$ 


\begin{tabular}{|c|c|c|c|c|c|c|}
\hline $78 \%$ & $17 \%$ & $86 \%$ & $15 \%$ & 66 & $41 \%$ & $100 \%$ \\
\hline $0 \%$ & $0 \%$ & $0 \%$ & $0 \%$ & 2324 & $66 \%$ & $96 \%$ \\
\hline $0 \%$ & $0 \%$ & $0 \%$ & $0 \%$ & 181 & $92 \%$ & $48 \%$ \\
\hline $0 \%$ & $0 \%$ & $0 \%$ & $0 \%$ & 0 & $0 \%$ & $0 \%$ \\
\hline $93 \%$ & $29 \%$ & $13 \%$ & $7 \%$ & 9 & $2 \%$ & $78 \%$ \\
\hline $0 \%$ & $0 \%$ & $0 \%$ & $0 \%$ & 0 & $0 \%$ & $0 \%$ \\
\hline $90 \%$ & $75 \%$ & $7 \%$ & $6 \%$ & 235 & $78 \%$ & $43 \%$ \\
\hline $3 \%$ & $24 \%$ & $5 \%$ & $0 \%$ & 80 & $59 \%$ & $23 \%$ \\
\hline $11 \%$ & $6 \%$ & $0 \%$ & $0 \%$ & 306 & $46 \%$ & $22 \%$ \\
\hline $2 \%$ & $62 \%$ & $54 \%$ & $1 \%$ & 61 & $64 \%$ & $59 \%$ \\
\hline $60 \%$ & $70 \%$ & $18 \%$ & $11 \%$ & 1246 & $73 \%$ & $93 \%$ \\
\hline $0 \%$ & $0 \%$ & $0 \%$ & $0 \%$ & 0 & $0 \%$ & $0 \%$ \\
\hline $93 \%$ & $93 \%$ & $73 \%$ & $69 \%$ & 170 & $89 \%$ & $98 \%$ \\
\hline $55 \%$ & $39 \%$ & $5 \%$ & $4 \%$ & 212 & $61 \%$ & $83 \%$ \\
\hline $100 \%$ & $96 \%$ & $99 \%$ & $95 \%$ & 22 & $81 \%$ & $100 \%$ \\
\hline $38 \%$ & $49 \%$ & $23 \%$ & $4 \%$ & 70 & $40 \%$ & $66 \%$ \\
\hline $26 \%$ & $21 \%$ & $0 \%$ & $0 \%$ & 5 & $38 \%$ & $60 \%$ \\
\hline $39 \%$ & $3 \%$ & $2 \%$ & $0 \%$ & 104 & $50 \%$ & $79 \%$ \\
\hline $9 \%$ & $3 \%$ & $1 \%$ & $0 \%$ & 56 & $31 \%$ & $13 \%$ \\
\hline $74 \%$ & $11 \%$ & $11 \%$ & $5 \%$ & 39 & $41 \%$ & $28 \%$ \\
\hline $0 \%$ & $28 \%$ & $24 \%$ & $0 \%$ & 3 & $38 \%$ & $33 \%$ \\
\hline $17 \%$ & $17 \%$ & $0 \%$ & $0 \%$ & 2 & $13 \%$ & $100 \%$ \\
\hline $73 \%$ & $47 \%$ & $29 \%$ & $16 \%$ & 4 & $21 \%$ & $75 \%$ \\
\hline $41 \%$ & $19 \%$ & $8 \%$ & $5 \%$ & 35 & $45 \%$ & $34 \%$ \\
\hline $16 \%$ & $12 \%$ & $0 \%$ & $0 \%$ & 2 & $7 \%$ & $50 \%$ \\
\hline $79 \%$ & $88 \%$ & $73 \%$ & $56 \%$ & 12 & $50 \%$ & $92 \%$ \\
\hline $66 \%$ & $47 \%$ & $16 \%$ & $10 \%$ & 8 & $33 \%$ & $38 \%$ \\
\hline $50 \%$ & $43 \%$ & $12 \%$ & $4 \%$ & 8 & $42 \%$ & $88 \%$ \\
\hline $67 \%$ & $36 \%$ & $12 \%$ & $4 \%$ & 11 & $58 \%$ & $82 \%$ \\
\hline $81 \%$ & $21 \%$ & $1 \%$ & $0 \%$ & 4 & $31 \%$ & $75 \%$ \\
\hline $59 \%$ & $41 \%$ & $5 \%$ & $5 \%$ & 5 & $42 \%$ & $80 \%$ \\
\hline $93 \%$ & $10 \%$ & $10 \%$ & $3 \%$ & 5 & $83 \%$ & $80 \%$ \\
\hline $63 \%$ & $26 \%$ & $26 \%$ & $6 \%$ & 4 & $67 \%$ & $75 \%$ \\
\hline $4 \%$ & $19 \%$ & $15 \%$ & $0 \%$ & 6 & $100 \%$ & $100 \%$ \\
\hline $0 \%$ & $0 \%$ & $0 \%$ & $0 \%$ & 0 & $0 \%$ & $0 \%$ \\
\hline $0 \%$ & $0 \%$ & $0 \%$ & $0 \%$ & 1 & $13 \%$ & $0 \%$ \\
\hline $99 \%$ & $9 \%$ & $2 \%$ & $1 \%$ & 29 & $31 \%$ & $100 \%$ \\
\hline $38 \%$ & $27 \%$ & $11 \%$ & $7 \%$ & 283 & $47 \%$ & $35 \%$ \\
\hline $41 \%$ & $45 \%$ & $29 \%$ & $13 \%$ & 2 & $2 \%$ & $100 \%$ \\
\hline $0 \%$ & $0 \%$ & $0 \%$ & $0 \%$ & 0 & $0 \%$ & $0 \%$ \\
\hline $0 \%$ & $0 \%$ & $0 \%$ & $0 \%$ & 0 & $0 \%$ & $0 \%$ \\
\hline $89 \%$ & $19 \%$ & $4 \%$ & $0 \%$ & 9 & $100 \%$ & $100 \%$ \\
\hline $91 \%$ & $87 \%$ & $11 \%$ & $11 \%$ & 580 & $31 \%$ & $76 \%$ \\
\hline $63 \%$ & $59 \%$ & $4 \%$ & $4 \%$ & 317 & $60 \%$ & $65 \%$ \\
\hline $84 \%$ & $89 \%$ & $6 \%$ & $6 \%$ & 1348 & $88 \%$ & $96 \%$ \\
\hline $34 \%$ & $32 \%$ & $3 \%$ & $1 \%$ & 150 & $53 \%$ & $69 \%$ \\
\hline $47 \%$ & $47 \%$ & $33 \%$ & $7 \%$ & 5 & $71 \%$ & $100 \%$ \\
\hline
\end{tabular}




\begin{tabular}{|c|c|c|c|c|c|c|}
\hline $7 \%$ & $23 \%$ & $8 \%$ & $3 \%$ & 300 & $70 \%$ & $22 \%$ \\
\hline $20 \%$ & $17 \%$ & $0 \%$ & $0 \%$ & 378 & $61 \%$ & $32 \%$ \\
\hline $90 \%$ & $82 \%$ & $45 \%$ & $42 \%$ & 220 & $76 \%$ & $45 \%$ \\
\hline $6 \%$ & $99 \%$ & $11 \%$ & $1 \%$ & 198 & $55 \%$ & $99 \%$ \\
\hline $67 \%$ & $50 \%$ & $0 \%$ & $0 \%$ & 2 & $67 \%$ & $100 \%$ \\
\hline $72 \%$ & $64 \%$ & $68 \%$ & $42 \%$ & 664 & $75 \%$ & $77 \%$ \\
\hline $99 \%$ & $98 \%$ & $29 \%$ & $28 \%$ & 153 & $13 \%$ & $90 \%$ \\
\hline $43 \%$ & $29 \%$ & $29 \%$ & $29 \%$ & 8 & $67 \%$ & $100 \%$ \\
\hline $6 \%$ & $4 \%$ & $1 \%$ & $1 \%$ & 40 & $93 \%$ & $95 \%$ \\
\hline $6 \%$ & $8 \%$ & $2 \%$ & $0 \%$ & 57 & $54 \%$ & $18 \%$ \\
\hline $85 \%$ & $69 \%$ & $0 \%$ & $0 \%$ & 2 & $3 \%$ & $100 \%$ \\
\hline $55 \%$ & $34 \%$ & $19 \%$ & $11 \%$ & 4 & $18 \%$ & $75 \%$ \\
\hline $100 \%$ & $50 \%$ & $50 \%$ & $50 \%$ & 3 & $18 \%$ & $33 \%$ \\
\hline $100 \%$ & $60 \%$ & $0 \%$ & $0 \%$ & 1 & $33 \%$ & $100 \%$ \\
\hline $50 \%$ & $50 \%$ & $50 \%$ & $0 \%$ & 0 & $0 \%$ & $0 \%$ \\
\hline $99 \%$ & $38 \%$ & $26 \%$ & $14 \%$ & 178 & $14 \%$ & $100 \%$ \\
\hline $10 \%$ & $2 \%$ & $1 \%$ & $0 \%$ & 64 & $46 \%$ & $94 \%$ \\
\hline $77 \%$ & $21 \%$ & $18 \%$ & $3 \%$ & 576 & $77 \%$ & $94 \%$ \\
\hline $0 \%$ & $0 \%$ & $0 \%$ & $0 \%$ & 0 & $0 \%$ & $0 \%$ \\
\hline $24 \%$ & $5 \%$ & $2 \%$ & $1 \%$ & 199 & $53 \%$ & $83 \%$ \\
\hline $0 \%$ & $0 \%$ & $0 \%$ & $0 \%$ & 12 & $4 \%$ & $25 \%$ \\
\hline $98 \%$ & $96 \%$ & $31 \%$ & $31 \%$ & 17 & $6 \%$ & $76 \%$ \\
\hline $36 \%$ & $36 \%$ & $9 \%$ & $9 \%$ & 6 & $67 \%$ & $33 \%$ \\
\hline $15 \%$ & $15 \%$ & $6 \%$ & $3 \%$ & 1 & $4 \%$ & $100 \%$ \\
\hline $71 \%$ & $60 \%$ & $13 \%$ & $9 \%$ & 16 & $22 \%$ & $69 \%$ \\
\hline $75 \%$ & $100 \%$ & $100 \%$ & $75 \%$ & 0 & $0 \%$ & $0 \%$ \\
\hline $90 \%$ & $76 \%$ & $9 \%$ & $8 \%$ & 388 & $38 \%$ & $77 \%$ \\
\hline $97 \%$ & $25 \%$ & $1 \%$ & $0 \%$ & 312 & $29 \%$ & $47 \%$ \\
\hline $79 \%$ & $79 \%$ & $34 \%$ & $33 \%$ & 22 & $48 \%$ & $68 \%$ \\
\hline $50 \%$ & $0 \%$ & $0 \%$ & $0 \%$ & 0 & $0 \%$ & $0 \%$ \\
\hline $36 \%$ & $30 \%$ & $20 \%$ & $17 \%$ & 32 & $30 \%$ & $9 \%$ \\
\hline $100 \%$ & $60 \%$ & $20 \%$ & $0 \%$ & 2 & $50 \%$ & $50 \%$ \\
\hline $46 \%$ & $7 \%$ & $0 \%$ & $0 \%$ & 2 & $5 \%$ & $100 \%$ \\
\hline
\end{tabular}


\% with CSF \% secondary \% primary oligoclonal progressive progressive \% progressive \% relapsing- \% with bands results MS $35 \%$ $14 \%$

$0 \%$

$0 \%$

$8 \%$

$7 \%$

$0 \%$

$0 \%$

$33 \%$

$0 \%$

$69 \%$

$0 \%$

$0 \%$

$32 \%$

$32 \%$

$0 \%$

$44 \%$

$71 \%$

$24 \%$

$0 \%$

$13 \%$

$29 \%$

$31 \%$

$43 \%$

$86 \%$

$0 \%$

$44 \%$

$32 \%$

$33 \%$

$75 \%$

$81 \%$

$41 \%$

$0 \%$

$0 \%$

$75 \%$

$0 \%$

$25 \%$

$71 \%$

$0 \%$

$1 \%$

$7 \%$

$0 \%$ MS

$2 \%$

$0 \%$

95\%

$8 \%$

$10 \%$

$14 \%$

$3 \%$

$4 \%$

$0 \%$

$17 \%$

$0 \%$

$0 \%$

$5 \%$

$7 \%$

$6 \%$

$7 \%$

$6 \%$

$2 \%$

$6 \%$

$2 \%$

$7 \%$

$4 \%$

$14 \%$

$2 \%$

9\%

$5 \%$

$7 \%$

$0 \%$

$7 \%$

$7 \%$

$6 \%$

$0 \%$

$0 \%$

$0 \%$

$3 \%$

$3 \%$

$7 \%$

$0 \%$

$7 \%$

$10 \%$

$0 \%$

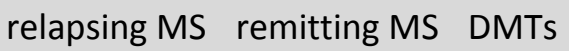

$66 \% \quad 46 \%$

$92 \%$

$0 \%$

$5 \%$

$92 \%$

$83 \%$

$46 \%$

$86 \%$

$69 \%$

$0 \%$

$83 \%$

$100 \%$

$88 \%$

$88 \%$

$84 \%$

$89 \%$

$86 \%$

$79 \%$

$89 \%$

$88 \%$

$96 \%$

$86 \%$

$79 \%$

$80 \%$

$92 \%$

$85 \%$

$90 \%$

$89 \%$

$0 \%$

$85 \%$

$80 \%$

$84 \%$

$100 \%$

$0 \%$

$92 \%$

$81 \%$

$79 \%$

$87 \%$

$0 \%$

$87 \%$

$75 \%$

$71 \%$
$\%$ of relapsing-

remitting with DMTs

\section{$85 \%$}

$78 \%$

$58 \%$

$0 \%$

$57 \%$

$71 \%$

$88 \%$

$45 \%$

$80 \%$

$70 \%$

$67 \%$

$42 \%$

$39 \%$

$74 \%$

$6 \%$

$62 \%$

$67 \%$

$53 \%$

$58 \%$

$64 \%$

$72 \%$

$74 \%$

$63 \%$

$80 \%$

$36 \%$

$66 \%$

$71 \%$

$71 \%$

$26 \%$

$48 \%$

$68 \%$

$100 \%$

$35 \%$

$83 \%$

$81 \%$

$68 \%$

$66 \%$

$44 \%$

$73 \%$

$49 \%$

$57 \%$
$100 \%$

$100 \%$

$0 \%$

$0 \%$

$0 \%$

$87 \%$

$0 \%$

$83 \%$

$85 \%$

$0 \%$

$100 \%$

$0 \%$

$89 \%$

$95 \%$

$89 \%$

$0 \%$

$82 \%$

$90 \%$

$100 \%$

$0 \%$

$98 \%$

$88 \%$

$91 \%$

$88 \%$

$97 \%$

$41 \%$

$100 \%$

$90 \%$

$0 \%$

$0 \%$

93\%

$84 \%$

$0 \%$

$0 \%$

$92 \%$

$92 \%$

$81 \%$

$75 \%$

$0 \%$

$82 \%$

$70 \%$

$0 \%$ 


\begin{tabular}{|c|c|c|c|c|c|c|}
\hline $8 \%$ & $13 \%$ & $7 \%$ & $1 \%$ & $79 \%$ & $45 \%$ & $53 \%$ \\
\hline $15 \%$ & $4 \%$ & $4 \%$ & $0 \%$ & $62 \%$ & $66 \%$ & $86 \%$ \\
\hline $31 \%$ & $1 \%$ & $1 \%$ & $1 \%$ & $62 \%$ & $93 \%$ & $0 \%$ \\
\hline $0 \%$ & $0 \%$ & $0 \%$ & $0 \%$ & $0 \%$ & $3 \%$ & $0 \%$ \\
\hline $0 \%$ & $4 \%$ & $5 \%$ & $1 \%$ & $92 \%$ & $74 \%$ & $96 \%$ \\
\hline $0 \%$ & $3 \%$ & $7 \%$ & $7 \%$ & $83 \%$ & $0 \%$ & $0 \%$ \\
\hline $51 \%$ & $14 \%$ & $6 \%$ & $0 \%$ & $74 \%$ & $70 \%$ & $94 \%$ \\
\hline $6 \%$ & $13 \%$ & $5 \%$ & $1 \%$ & $84 \%$ & $73 \%$ & $92 \%$ \\
\hline $5 \%$ & $10 \%$ & $4 \%$ & $1 \%$ & $75 \%$ & $52 \%$ & $87 \%$ \\
\hline $44 \%$ & $4 \%$ & $16 \%$ & $0 \%$ & $75 \%$ & $61 \%$ & $100 \%$ \\
\hline $76 \%$ & $17 \%$ & $8 \%$ & $1 \%$ & $85 \%$ & $65 \%$ & $83 \%$ \\
\hline $0 \%$ & $0 \%$ & $0 \%$ & $0 \%$ & $100 \%$ & $0 \%$ & $0 \%$ \\
\hline $56 \%$ & $10 \%$ & $12 \%$ & $2 \%$ & $66 \%$ & $44 \%$ & $63 \%$ \\
\hline $8 \%$ & $15 \%$ & $11 \%$ & $1 \%$ & $76 \%$ & $41 \%$ & $62 \%$ \\
\hline $82 \%$ & $0 \%$ & $0 \%$ & $0 \%$ & $96 \%$ & $81 \%$ & $88 \%$ \\
\hline $3 \%$ & $0 \%$ & $1 \%$ & $1 \%$ & $47 \%$ & $21 \%$ & $100 \%$ \\
\hline $20 \%$ & $0 \%$ & $0 \%$ & $0 \%$ & $31 \%$ & $8 \%$ & $0 \%$ \\
\hline $41 \%$ & $24 \%$ & $14 \%$ & $0 \%$ & $79 \%$ & $42 \%$ & $78 \%$ \\
\hline $2 \%$ & $11 \%$ & $6 \%$ & $1 \%$ & $87 \%$ & $22 \%$ & $94 \%$ \\
\hline $69 \%$ & $9 \%$ & $3 \%$ & $1 \%$ & $91 \%$ & $59 \%$ & $100 \%$ \\
\hline $0 \%$ & $0 \%$ & $38 \%$ & $25 \%$ & $38 \%$ & $0 \%$ & $0 \%$ \\
\hline $0 \%$ & $0 \%$ & $0 \%$ & $0 \%$ & $0 \%$ & $0 \%$ & $0 \%$ \\
\hline $25 \%$ & $0 \%$ & $0 \%$ & $0 \%$ & $100 \%$ & $100 \%$ & $100 \%$ \\
\hline $37 \%$ & $1 \%$ & $0 \%$ & $0 \%$ & $100 \%$ & $96 \%$ & $100 \%$ \\
\hline $0 \%$ & $0 \%$ & $7 \%$ & $4 \%$ & $86 \%$ & $100 \%$ & $0 \%$ \\
\hline $25 \%$ & $0 \%$ & $4 \%$ & $4 \%$ & $88 \%$ & $100 \%$ & $100 \%$ \\
\hline $38 \%$ & $0 \%$ & $0 \%$ & $0 \%$ & $96 \%$ & $96 \%$ & $100 \%$ \\
\hline $13 \%$ & $5 \%$ & $0 \%$ & $0 \%$ & $84 \%$ & $95 \%$ & $0 \%$ \\
\hline $18 \%$ & $0 \%$ & $0 \%$ & $0 \%$ & $95 \%$ & $89 \%$ & $0 \%$ \\
\hline $0 \%$ & $0 \%$ & $0 \%$ & $0 \%$ & $100 \%$ & $100 \%$ & $0 \%$ \\
\hline $0 \%$ & $0 \%$ & $0 \%$ & $0 \%$ & $92 \%$ & $100 \%$ & $100 \%$ \\
\hline $0 \%$ & $0 \%$ & $0 \%$ & $0 \%$ & $100 \%$ & $100 \%$ & $100 \%$ \\
\hline $0 \%$ & $0 \%$ & $0 \%$ & $0 \%$ & $83 \%$ & $67 \%$ & $0 \%$ \\
\hline $67 \%$ & $0 \%$ & $0 \%$ & $0 \%$ & $83 \%$ & $100 \%$ & $100 \%$ \\
\hline $0 \%$ & $0 \%$ & $0 \%$ & $0 \%$ & $0 \%$ & $0 \%$ & $0 \%$ \\
\hline $0 \%$ & $13 \%$ & $25 \%$ & $13 \%$ & $75 \%$ & $50 \%$ & $0 \%$ \\
\hline $3 \%$ & $7 \%$ & $3 \%$ & $2 \%$ & $88 \%$ & $96 \%$ & $100 \%$ \\
\hline $2 \%$ & $6 \%$ & $8 \%$ & $3 \%$ & $65 \%$ & $9 \%$ & $46 \%$ \\
\hline $0 \%$ & $3 \%$ & $6 \%$ & $6 \%$ & $69 \%$ & $9 \%$ & $0 \%$ \\
\hline $0 \%$ & $0 \%$ & $2 \%$ & $1 \%$ & $98 \%$ & $38 \%$ & $0 \%$ \\
\hline $0 \%$ & $0 \%$ & $2 \%$ & $0 \%$ & $97 \%$ & $1 \%$ & $100 \%$ \\
\hline $22 \%$ & $0 \%$ & $0 \%$ & $0 \%$ & $56 \%$ & $22 \%$ & $0 \%$ \\
\hline $26 \%$ & $7 \%$ & $7 \%$ & $4 \%$ & $80 \%$ & $52 \%$ & $81 \%$ \\
\hline $26 \%$ & $21 \%$ & $5 \%$ & $1 \%$ & $83 \%$ & $56 \%$ & $75 \%$ \\
\hline $81 \%$ & $11 \%$ & $4 \%$ & $2 \%$ & $90 \%$ & $86 \%$ & $95 \%$ \\
\hline $10 \%$ & $12 \%$ & $8 \%$ & $6 \%$ & $84 \%$ & $46 \%$ & $66 \%$ \\
\hline $60 \%$ & $0 \%$ & $14 \%$ & $14 \%$ & $86 \%$ & $57 \%$ & $0 \%$ \\
\hline
\end{tabular}




\begin{tabular}{|c|c|c|c|c|c|c|}
\hline $6 \%$ & $7 \%$ & $3 \%$ & $2 \%$ & $92 \%$ & $61 \%$ & $64 \%$ \\
\hline $26 \%$ & $13 \%$ & $4 \%$ & $1 \%$ & $76 \%$ & $47 \%$ & $85 \%$ \\
\hline $18 \%$ & $10 \%$ & $6 \%$ & $2 \%$ & $79 \%$ & $78 \%$ & $92 \%$ \\
\hline $76 \%$ & $17 \%$ & $8 \%$ & $1 \%$ & $78 \%$ & $63 \%$ & $88 \%$ \\
\hline $100 \%$ & $0 \%$ & $0 \%$ & $0 \%$ & $100 \%$ & $0 \%$ & $0 \%$ \\
\hline $71 \%$ & $14 \%$ & $7 \%$ & $2 \%$ & $82 \%$ & $57 \%$ & $76 \%$ \\
\hline $16 \%$ & $9 \%$ & $3 \%$ & $0 \%$ & $85 \%$ & $50 \%$ & $98 \%$ \\
\hline $13 \%$ & $0 \%$ & $0 \%$ & $0 \%$ & $42 \%$ & $25 \%$ & $0 \%$ \\
\hline $48 \%$ & $0 \%$ & $0 \%$ & $0 \%$ & $58 \%$ & $35 \%$ & $75 \%$ \\
\hline $32 \%$ & $6 \%$ & $8 \%$ & $5 \%$ & $75 \%$ & $50 \%$ & $76 \%$ \\
\hline $50 \%$ & $6 \%$ & $1 \%$ & $0 \%$ & $91 \%$ & $1 \%$ & $0 \%$ \\
\hline $25 \%$ & $0 \%$ & $14 \%$ & $5 \%$ & $68 \%$ & $32 \%$ & $0 \%$ \\
\hline $0 \%$ & $0 \%$ & $0 \%$ & $0 \%$ & $94 \%$ & $6 \%$ & $0 \%$ \\
\hline $100 \%$ & $0 \%$ & $33 \%$ & $0 \%$ & $33 \%$ & $0 \%$ & $0 \%$ \\
\hline $0 \%$ & $0 \%$ & $100 \%$ & $100 \%$ & $0 \%$ & $100 \%$ & $0 \%$ \\
\hline $85 \%$ & $28 \%$ & $19 \%$ & $4 \%$ & $73 \%$ & $32 \%$ & $83 \%$ \\
\hline $0 \%$ & $9 \%$ & $10 \%$ & $6 \%$ & $87 \%$ & $34 \%$ & $0 \%$ \\
\hline $14 \%$ & $17 \%$ & $16 \%$ & $3 \%$ & $73 \%$ & $66 \%$ & $82 \%$ \\
\hline $0 \%$ & $33 \%$ & $0 \%$ & $0 \%$ & $95 \%$ & $17 \%$ & $40 \%$ \\
\hline $17 \%$ & $7 \%$ & $9 \%$ & $6 \%$ & $83 \%$ & $53 \%$ & $85 \%$ \\
\hline $0 \%$ & $23 \%$ & $15 \%$ & $7 \%$ & $80 \%$ & $8 \%$ & $71 \%$ \\
\hline $0 \%$ & $11 \%$ & $9 \%$ & $5 \%$ & $83 \%$ & $81 \%$ & $96 \%$ \\
\hline $33 \%$ & $0 \%$ & $0 \%$ & $0 \%$ & $89 \%$ & $78 \%$ & $0 \%$ \\
\hline $0 \%$ & $19 \%$ & $8 \%$ & $0 \%$ & $73 \%$ & $92 \%$ & $100 \%$ \\
\hline $6 \%$ & $8 \%$ & $12 \%$ & $8 \%$ & $77 \%$ & $55 \%$ & $0 \%$ \\
\hline $0 \%$ & $0 \%$ & $0 \%$ & $0 \%$ & $0 \%$ & $33 \%$ & $0 \%$ \\
\hline $26 \%$ & $8 \%$ & $6 \%$ & $3 \%$ & $77 \%$ & $49 \%$ & $83 \%$ \\
\hline $64 \%$ & $6 \%$ & $6 \%$ & $2 \%$ & $76 \%$ & $61 \%$ & $95 \%$ \\
\hline $18 \%$ & $4 \%$ & $7 \%$ & $2 \%$ & $85 \%$ & $65 \%$ & $100 \%$ \\
\hline $0 \%$ & $0 \%$ & $0 \%$ & $0 \%$ & $100 \%$ & $0 \%$ & $0 \%$ \\
\hline $50 \%$ & $2 \%$ & $4 \%$ & $2 \%$ & $89 \%$ & $47 \%$ & $100 \%$ \\
\hline $100 \%$ & $0 \%$ & $0 \%$ & $0 \%$ & $75 \%$ & $0 \%$ & $0 \%$ \\
\hline $100 \%$ & $0 \%$ & $0 \%$ & $0 \%$ & $0 \%$ & $0 \%$ & \\
\hline
\end{tabular}


Patients treated with:

mean \% o recorded time on DMTs
$\%$ of recorded $\%$ of recorded

time on DMTs time on DMTs

$46 \%$

$39 \%$

$38 \%$

$74 \%$

$80 \%$

$0 \%$

$62 \%$

$72 \%$

$78 \%$

$55 \%$

$75 \%$

$72 \%$

$82 \%$

$57 \%$

$73 \%$

$55 \%$

$51 \%$

$57 \%$

$62 \%$

$83 \%$

$76 \%$

$76 \%$

$64 \%$

$68 \%$

$55 \%$

$75 \%$

$21 \%$

$84 \%$

$60 \%$

$74 \%$

$55 \%$

$65 \%$

$64 \%$

$32 \%$

$34 \%$

$71 \%$

$68 \%$

$52 \%$

$42 \%$

$72 \%$

$56 \%$

$41 \%$

$43 \%$
$0 \%$

$45 \%$

$43 \%$

$39 \%$

$44 \%$

$43 \%$

$43 \%$

$50 \%$

$43 \%$

$43 \%$

$42 \%$

$19 \%$

$44 \%$

$44 \%$

$48 \%$

$47 \%$

$44 \%$

$43 \%$

$42 \%$

$44 \%$

$40 \%$

$39 \%$

$45 \%$

$40 \%$

$43 \%$

$40 \%$

$47 \%$

$44 \%$

$0 \%$

$45 \%$

$35 \%$

$42 \%$

$42 \%$

$42 \%$

$48 \%$

$41 \%$

$42 \%$

$36 \%$

$\mathrm{coV}$

$71 \%$

$43 \%$

$52 \%$

$92 \%$

$0 \%$

$72 \%$

$60 \%$

$50 \%$

$79 \%$

$58 \%$

$61 \%$

$61 \%$

$77 \%$

$60 \%$

$77 \%$

$36 \%$

$76 \%$

$71 \%$

$57 \%$

$63 \%$

$58 \%$

$67 \%$

$62 \%$

$80 \%$

$54 \%$

$187 \%$

$53 \%$

$67 \%$

$58 \%$

$72 \%$

$71 \%$

$68 \%$

$0 \%$

$131 \%$

$50 \%$

$62 \%$

$82 \%$

$100 \%$

$67 \%$

$73 \%$

$101 \%$

$84 \%$
Pegylated

nterferon Interferon $\beta$-Interferon Interferon $\beta$ - Interferon $\beta$-1a IM 1a IM $\quad \beta$-1b $1 b$ (generic) $\beta$-1asc

11

48

67

6

0

0

0

0

0

0

0

132

2

73

33

37

139

39

65

12

7

10

0
19

16

5

220

132

110
116

2

2
238
116
2

0

0

10

1

0

19

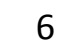

11

37

5

91

43

1

1

8

88

19

8

1

0

0

0

$\begin{array}{rr}0 & 6 \\ 0 & 36\end{array}$

$0 \quad 21$

$0 \quad 55$

$0 \quad 88$

$\begin{array}{ll}0 & 19\end{array}$

$\begin{array}{ll}0 & 7\end{array}$

0

0

0

0

0

0

$0 \quad 18$

$0 \quad 8$

$0 \quad 19$

$0 \quad 30$

$0 \quad 102$

$\begin{array}{ll}0 & 48\end{array}$

$0 \quad 52$

$0 \quad 21$

$0 \quad 16$

$0 \quad 6$

$0 \quad 6$

03

$0 \quad 0$

$0 \quad 0$

$0 \quad 161$

$0 \quad 0$

$0 \quad 5$

$0 \quad 6$

$0 \quad 44$

$\begin{array}{ll}0 & 18\end{array}$

$\begin{array}{ll}0 & 372\end{array}$

$0 \quad 4$

$0 \quad 299$

$0 \quad 153$

1 


\begin{tabular}{|c|c|c|c|c|c|c|c|}
\hline $22 \%$ & $29 \%$ & $134 \%$ & 18 & 0 & 54 & 0 & 20 \\
\hline $60 \%$ & $44 \%$ & $73 \%$ & 994 & 0 & 603 & 0 & 805 \\
\hline $88 \%$ & $26 \%$ & $30 \%$ & 41 & 0 & 48 & 0 & 47 \\
\hline $58 \%$ & $18 \%$ & $30 \%$ & 2 & 0 & 3 & 0 & 8 \\
\hline $55 \%$ & $40 \%$ & $73 \%$ & 118 & 0 & 88 & 0 & 167 \\
\hline $0 \%$ & $0 \%$ & $0 \%$ & 0 & 0 & 0 & 0 & 0 \\
\hline $66 \%$ & $42 \%$ & $63 \%$ & 28 & 0 & 76 & 0 & 99 \\
\hline $66 \%$ & $41 \%$ & $63 \%$ & 19 & 0 & 28 & 0 & 39 \\
\hline $62 \%$ & $43 \%$ & $70 \%$ & 118 & 0 & 164 & 0 & 93 \\
\hline $68 \%$ & $44 \%$ & $64 \%$ & 17 & 0 & 7 & 0 & 30 \\
\hline $58 \%$ & $42 \%$ & $73 \%$ & 231 & 0 & 364 & 0 & 397 \\
\hline $0 \%$ & $0 \%$ & $0 \%$ & 0 & 0 & 0 & 0 & 0 \\
\hline $38 \%$ & $41 \%$ & $108 \%$ & 27 & 0 & 13 & 0 & 24 \\
\hline $33 \%$ & $38 \%$ & $117 \%$ & 67 & 0 & 39 & 0 & 29 \\
\hline $67 \%$ & $34 \%$ & $51 \%$ & 3 & 0 & 9 & 0 & 5 \\
\hline $40 \%$ & $30 \%$ & $74 \%$ & 14 & 0 & 9 & 0 & 16 \\
\hline $9 \%$ & $26 \%$ & $280 \%$ & 0 & 0 & 0 & 0 & 0 \\
\hline $32 \%$ & $42 \%$ & $129 \%$ & 32 & 0 & 10 & 0 & 41 \\
\hline $60 \%$ & $36 \%$ & $59 \%$ & 27 & 0 & 9 & 0 & 29 \\
\hline $57 \%$ & $39 \%$ & $68 \%$ & 22 & 0 & 13 & 0 & 19 \\
\hline $0 \%$ & $0 \%$ & $0 \%$ & 0 & 0 & 0 & 0 & 1 \\
\hline $0 \%$ & $0 \%$ & $0 \%$ & 0 & 0 & 0 & 0 & 0 \\
\hline $86 \%$ & $16 \%$ & $19 \%$ & 6 & 0 & 2 & 0 & 0 \\
\hline $86 \%$ & $22 \%$ & $26 \%$ & 9 & 0 & 12 & 0 & 24 \\
\hline $96 \%$ & $22 \%$ & $23 \%$ & 3 & 0 & 0 & 0 & 0 \\
\hline $87 \%$ & $17 \%$ & $20 \%$ & 11 & 0 & 1 & 0 & 2 \\
\hline $92 \%$ & $21 \%$ & $22 \%$ & 0 & 0 & 1 & 0 & 1 \\
\hline $95 \%$ & $28 \%$ & $29 \%$ & 2 & 0 & 3 & 0 & 1 \\
\hline $80 \%$ & $36 \%$ & $45 \%$ & 4 & 0 & 2 & 0 & 1 \\
\hline $98 \%$ & $4 \%$ & $4 \%$ & 3 & 0 & 1 & 0 & 0 \\
\hline $83 \%$ & $23 \%$ & $28 \%$ & 5 & 0 & 3 & 0 & 3 \\
\hline $94 \%$ & $6 \%$ & $7 \%$ & 1 & 0 & 0 & 0 & 0 \\
\hline $99 \%$ & $51 \%$ & $52 \%$ & 1 & 0 & 1 & 0 & 0 \\
\hline $63 \%$ & $17 \%$ & $27 \%$ & 2 & 0 & 1 & 0 & 3 \\
\hline $0 \%$ & $0 \%$ & $0 \%$ & 0 & 0 & 0 & 0 & 0 \\
\hline $21 \%$ & $48 \%$ & $232 \%$ & 1 & 0 & 2 & 0 & 1 \\
\hline $85 \%$ & $27 \%$ & $32 \%$ & 22 & 0 & 36 & 0 & 29 \\
\hline $20 \%$ & $21 \%$ & $104 \%$ & 35 & 0 & 3 & 0 & 20 \\
\hline $40 \%$ & $24 \%$ & $60 \%$ & 16 & 0 & 0 & 0 & 7 \\
\hline $76 \%$ & $47 \%$ & $62 \%$ & 469 & 0 & 357 & 0 & 258 \\
\hline $23 \%$ & $7 \%$ & $30 \%$ & 120 & 0 & 102 & 13 & 50 \\
\hline $15 \%$ & $27 \%$ & $173 \%$ & 1 & 0 & 0 & 0 & 0 \\
\hline $42 \%$ & $39 \%$ & $94 \%$ & 302 & 0 & 200 & 0 & 407 \\
\hline $47 \%$ & $40 \%$ & $86 \%$ & 82 & 0 & 70 & 0 & 180 \\
\hline $64 \%$ & $34 \%$ & $54 \%$ & 465 & 0 & 218 & 0 & 850 \\
\hline $43 \%$ & $41 \%$ & $95 \%$ & 44 & 0 & 24 & 0 & 68 \\
\hline 79\% & $50 \%$ & $64 \%$ & 1 & 0 & 1 & 0 & 2 \\
\hline
\end{tabular}




$\begin{array}{rrrrrrrr}33 \% & 40 \% & 120 \% & 95 & 0 & 52 & 0 & 62 \\ 57 \% & 43 \% & 76 \% & 151 & 4 & 96 & 0 & 92 \\ 69 \% & 39 \% & 56 \% & 101 & 0 & 9 & 0 & 116 \\ 60 \% & 43 \% & 71 \% & 86 & 0 & 51 & 0 & 105 \\ 0 \% & 0 \% & 0 \% & 0 & 0 & 0 & 0 & 0 \\ 32 \% & 37 \% & 117 \% & 129 & 0 & 66 & 0 & 205 \\ 71 \% & 45 \% & 63 \% & 176 & 0 & 198 & 0 & 123 \\ 46 \% & 45 \% & 97 \% & 0 & 0 & 0 & 0 & 4 \\ 22 \% & 31 \% & 142 \% & 5 & 0 & 1 & 0 & 7 \\ 63 \% & 46 \% & 74 \% & 45 & 0 & 26 & 0 & 1 \\ 11 \% & 11 \% & 100 \% & 30 & 0 & 5 & 0 & 4 \\ 45 \% & 40 \% & 89 \% & 0 & 0 & 8 & 0 & 2 \\ 21 \% & 24 \% & 117 \% & 0 & 0 & 0 & 0 & 2 \\ 0 \% & 0 \% & 0 \% & 0 & 0 & 0 & 0 & 0 \\ 2 \% & 0 \% & 0 \% & 0 & 0 & 0 & 0 & 0 \\ 48 \% & 38 \% & 78 \% & 116 & 0 & 119 & 0 & 231 \\ 33 \% & 38 \% & 115 \% & 10 & 0 & 24 & 0 & 13 \\ 35 \% & 38 \% & 108 \% & 112 & 1 & 140 & 0 & 211 \\ 46 \% & 35 \% & 77 \% & 24 & 0 & 18 & 0 & 29 \\ 57 \% & 43 \% & 75 \% & 73 & 0 & 37 & 0 & 89 \\ 51 \% & 25 \% & 49 \% & 24 & 0 & 17 & 0 & 10 \\ 69 \% & 39 \% & 56 \% & 96 & 0 & 74 & 0 & 115 \\ 72 \% & 42 \% & 58 \% & 2 & 0 & 2 & 0 & 2 \\ 71 \% & 31 \% & 44 \% & 0 & 0 & 22 & 0 & 0 \\ 82 \% & 53 \% & 65 \% & 5 & 0 & 17 & 0 & 17 \\ 100 \% & 58 \% & 58 \% & 1 & 0 & 0 & 0 & 0 \\ 50 \% & 43 \% & 86 \% & 87 & 0 & 128 & 0 & 224 \\ 67 \% & 45 \% & 67 \% & 235 & 0 & 169 & 0 & 216 \\ 56 \% & 41 \% & 73 \% & 13 & 0 & 9 & 0 & 8 \\ 0 \% & 0 \% & 0 \% & 0 & 0 & 0 & 0 & 0 \\ 80 \% & 50 \% & 62 \% & 23 & 0 & 5 & 0 & 12 \\ 0 \% & 0 \% & 0 \% & 0 & 0 & 0 & 0 & 1 \\ 0 \% & 0 \% & 0 \% & 0 & 0 & 0 & 0 & 0\end{array}$




\begin{tabular}{|c|c|c|c|c|c|c|c|c|c|}
\hline $\begin{array}{l}\text { Interferon } \beta \\
\text { 1a SC } \\
\text { (generic) }\end{array}$ & $\begin{array}{l}\text { Glatiramer } \\
\text { Acetate }\end{array}$ & & $\begin{array}{l}\text { Glatiramer } \\
\text { Acetate } \\
\text { (generic) }\end{array}$ & $\begin{array}{l}\text { Glatiramer } \\
\text { Acetate } \\
40 \mathrm{mg}\end{array}$ & & Teriflunomide & $\begin{array}{l}\text { Dimethyl } \\
\text { Fumarate }\end{array}$ & Fingolimod & Cladribine \\
\hline & 0 & 43 & & 0 & 0 & 0 & 0 & 1 & - 0 \\
\hline & 0 & 24 & & 0 & 0 & 0 & 0 & 0 & 0 \\
\hline & 0 & 4 & & 0 & 0 & 0 & 0 & 0 & 0 \\
\hline & 0 & 0 & & 0 & 0 & 0 & 0 & 0 & 0 \\
\hline & 0 & 0 & & 0 & 0 & 0 & 0 & 0 & 0 \\
\hline & 0 & 33 & & 0 & 0 & 1 & 0 & 14 & 0 \\
\hline & 0 & 0 & & 0 & 0 & 0 & 0 & 0 & 0 \\
\hline & 0 & 3 & & 0 & 0 & 0 & 0 & 0 & 0 \\
\hline & 0 & 18 & & 0 & 0 & 0 & 0 & 3 & 0 \\
\hline & 0 & 1 & & 0 & 0 & 0 & 0 & 0 & 0 \\
\hline & 0 & 2 & & 0 & 0 & 2 & 0 & 4 & 0 \\
\hline & 0 & 1 & & 0 & 0 & 0 & 0 & 0 & 0 \\
\hline & 0 & 9 & & 0 & 0 & 1 & 4 & 22 & 0 \\
\hline & 0 & 64 & & 0 & 0 & 0 & 9 & 40 & 1 \\
\hline & 0 & 122 & & 0 & 5 & 26 & 95 & 322 & 7 \\
\hline & 0 & 1 & & 0 & 0 & 0 & 0 & 0 & 0 \\
\hline & 0 & 128 & & 0 & 0 & 15 & 60 & 113 & 6 \\
\hline & 0 & 22 & & 0 & 0 & 4 & 3 & 14 & 4 \\
\hline & 0 & 15 & & 0 & 0 & 3 & 5 & 17 & 0 \\
\hline & 0 & 25 & & 0 & 0 & 22 & 40 & 55 & 0 \\
\hline & 0 & 47 & & 0 & 0 & 4 & 35 & 162 & 11 \\
\hline & 0 & 120 & & 0 & 0 & 8 & 63 & 314 & 22 \\
\hline & 0 & 43 & & 0 & 0 & 6 & 37 & 60 & 2 \\
\hline & 0 & 87 & & 0 & 0 & 5 & 19 & 78 & 6 \\
\hline & 0 & 109 & & 0 & 0 & 30 & 35 & 116 & 6 \\
\hline & 0 & 16 & & 0 & 0 & 19 & 19 & 29 & 0 \\
\hline & 0 & 10 & & 0 & 0 & 1 & 6 & 60 & 1 \\
\hline & 0 & 4 & & 0 & 0 & 2 & 0 & 19 & 0 \\
\hline & 0 & 0 & & 0 & 0 & 2 & 7 & 7 & 0 \\
\hline & 0 & 1 & & 0 & 0 & 1 & 2 & 6 & 0 \\
\hline & 0 & 12 & & 0 & 0 & 4 & 3 & 13 & 0 \\
\hline 0 & 0 & 104 & & 0 & 0 & 41 & 17 & 59 & 0 \\
\hline & 0 & 1 & & 0 & 0 & 0 & 0 & 0 & 0 \\
\hline & 0 & 9 & & 0 & 0 & 0 & 0 & 0 & 0 \\
\hline & 0 & 33 & & 0 & 0 & 1 & 0 & 10 & 0 \\
\hline & 0 & 69 & & 0 & 0 & 28 & 28 & 43 & 0 \\
\hline & 0 & 13 & & 0 & 0 & 0 & 0 & 7 & 0 \\
\hline & 0 & 343 & & 0 & 0 & 142 & 59 & 97 & 0 \\
\hline 0 & 0 & 2 & & 0 & 0 & 0 & 0 & 0 & 0 \\
\hline & 0 & 267 & & 0 & 0 & 47 & 50 & 103 & 0 \\
\hline 0 & 0 & 238 & & 0 & 0 & 55 & 56 & 96 & 0 \\
\hline & 0 & 0 & & 0 & 0 & 0 & 0 & 1 & 0 \\
\hline
\end{tabular}




\begin{tabular}{|c|c|c|c|c|c|c|}
\hline 5 & 0 & 0 & 0 & 0 & 0 & 0 \\
\hline 591 & 0 & 41 & 61 & 59 & 372 & 0 \\
\hline 63 & 0 & 0 & 3 & 0 & 14 & 0 \\
\hline 5 & 0 & 0 & 0 & 0 & 0 & 0 \\
\hline 85 & 0 & 0 & 2 & 1 & 8 & 0 \\
\hline 0 & 0 & 0 & 0 & 0 & 0 & 0 \\
\hline 51 & 0 & 0 & 0 & 6 & 32 & 0 \\
\hline 40 & 0 & 0 & 2 & 0 & 22 & 0 \\
\hline 98 & 0 & 0 & 0 & 0 & 0 & 0 \\
\hline 19 & 0 & 0 & 0 & 17 & 1 & 0 \\
\hline 274 & 0 & 0 & 29 & 55 & 269 & 0 \\
\hline 0 & 0 & 0 & 0 & 0 & 0 & 0 \\
\hline 18 & 0 & 0 & 2 & 17 & 15 & 0 \\
\hline 20 & 0 & 0 & 2 & 12 & 18 & 0 \\
\hline 11 & 0 & 0 & 5 & 4 & 5 & 0 \\
\hline 9 & 0 & 0 & 0 & 0 & 0 & 0 \\
\hline 1 & 0 & 0 & 0 & 0 & 0 & 0 \\
\hline 18 & 0 & 0 & 0 & 0 & 0 & 0 \\
\hline 12 & 0 & 0 & 0 & 2 & 2 & 0 \\
\hline 18 & 0 & 0 & 1 & 12 & 8 & 0 \\
\hline 0 & 0 & 0 & 0 & 0 & 0 & 0 \\
\hline 0 & 0 & 0 & 0 & 0 & 0 & 0 \\
\hline 7 & 0 & 0 & 0 & 0 & 1 & 0 \\
\hline 32 & 0 & 0 & 0 & 0 & 0 & 0 \\
\hline 0 & 0 & 0 & 0 & 0 & 1 & 0 \\
\hline 4 & 0 & 0 & 0 & 0 & 3 & 0 \\
\hline 2 & 0 & 0 & 0 & 0 & 7 & 0 \\
\hline 4 & 0 & 0 & 0 & 0 & 0 & 0 \\
\hline 1 & 0 & 0 & 0 & 0 & 0 & 0 \\
\hline 0 & 0 & 0 & 0 & 0 & 0 & 0 \\
\hline 5 & 0 & 0 & 0 & 0 & 2 & 0 \\
\hline 1 & 0 & 0 & 0 & 0 & 0 & 0 \\
\hline 0 & 0 & 0 & 0 & 0 & 0 & 0 \\
\hline 2 & 0 & 0 & 0 & 1 & 0 & 0 \\
\hline 1 & 0 & 0 & 0 & 0 & 0 & 0 \\
\hline 1 & 0 & 0 & 0 & 0 & 0 & 0 \\
\hline 24 & 0 & 0 & 0 & 0 & 0 & 0 \\
\hline 3 & 0 & 0 & 0 & 0 & 0 & 0 \\
\hline 9 & 0 & 0 & 0 & 0 & 0 & 0 \\
\hline 50 & 0 & 0 & 0 & 0 & 76 & 0 \\
\hline 3 & 5 & 0 & 0 & 0 & 1 & 0 \\
\hline 1 & 0 & 0 & 0 & 0 & 0 & 0 \\
\hline 297 & 0 & 0 & 38 & 32 & 191 & 0 \\
\hline 89 & 0 & 0 & 6 & 13 & 24 & 0 \\
\hline 295 & 0 & 0 & 6 & 0 & 116 & 1 \\
\hline 41 & 0 & 0 & 1 & 0 & 1 & 1 \\
\hline 2 & 0 & 0 & 0 & 0 & 0 & 0 \\
\hline
\end{tabular}

http://mc.manuscriptcentral.com/multiple-sclerosis 


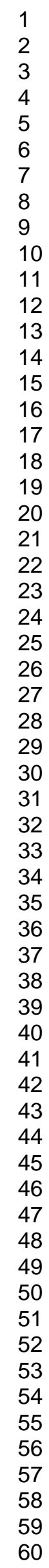

\begin{tabular}{|c|c|c|c|c|c|c|c|}
\hline 0 & 137 & 0 & 0 & 1 & 0 & 5 & 0 \\
\hline 0 & 107 & 0 & 0 & 1 & 33 & 25 & 0 \\
\hline 0 & 58 & 0 & 0 & 3 & 3 & 49 & 0 \\
\hline 0 & 64 & 0 & 0 & 4 & 7 & 39 & 0 \\
\hline 0 & 0 & 0 & 0 & 1 & 0 & 0 & 0 \\
\hline 0 & 267 & 0 & 0 & 1 & 3 & 43 & 0 \\
\hline 0 & 10 & 0 & 0 & 44 & 36 & 230 & 0 \\
\hline 0 & 0 & 0 & 0 & 0 & 0 & 0 & 0 \\
\hline 0 & 2 & 0 & 0 & 0 & 0 & 0 & 0 \\
\hline 0 & 0 & 0 & 0 & 0 & 0 & 0 & 0 \\
\hline 0 & 5 & 0 & 0 & 0 & 0 & 0 & 0 \\
\hline 0 & 0 & 0 & 0 & 0 & 0 & 0 & 0 \\
\hline 0 & 0 & 0 & 0 & 0 & 0 & 0 & 0 \\
\hline 0 & 0 & 0 & 0 & 0 & 0 & 0 & 0 \\
\hline 0 & 1 & 0 & 0 & 0 & 0 & 0 & 0 \\
\hline 0 & 129 & 0 & 0 & 0 & 0 & 24 & 0 \\
\hline 0 & 9 & 0 & 0 & 0 & 0 & 0 & 0 \\
\hline 0 & 145 & 0 & 1 & 7 & 12 & 32 & 0 \\
\hline 0 & 19 & 0 & 0 & 0 & 0 & 0 & 0 \\
\hline 0 & 99 & 0 & 0 & 6 & 2 & 24 & 0 \\
\hline 0 & 12 & 0 & 0 & 0 & 9 & 9 & 0 \\
\hline 0 & 31 & 0 & 0 & 0 & 0 & 1 & 0 \\
\hline 0 & 1 & 0 & 0 & 0 & 0 & 0 & 0 \\
\hline 0 & 0 & 0 & 0 & 0 & 0 & 0 & 0 \\
\hline 0 & 0 & 0 & 0 & 0 & 0 & 8 & 0 \\
\hline 0 & 0 & 0 & 0 & 0 & 0 & 0 & 0 \\
\hline 0 & 159 & 0 & 0 & 34 & 18 & 69 & 0 \\
\hline 0 & 190 & 0 & 0 & 20 & 22 & 137 & 2 \\
\hline 0 & 7 & 0 & 0 & 0 & 0 & 6 & 0 \\
\hline 0 & 0 & 0 & 0 & 0 & 0 & 0 & 0 \\
\hline 0 & 33 & 0 & 0 & 0 & 1 & 5 & 0 \\
\hline 0 & 0 & 0 & 0 & 0 & 0 & 0 & 0 \\
\hline 0 & 0 & 0 & 0 & 0 & 0 & 0 & 0 \\
\hline
\end{tabular}

http://mc.manuscriptcentral.com/multiple-sclerosis 


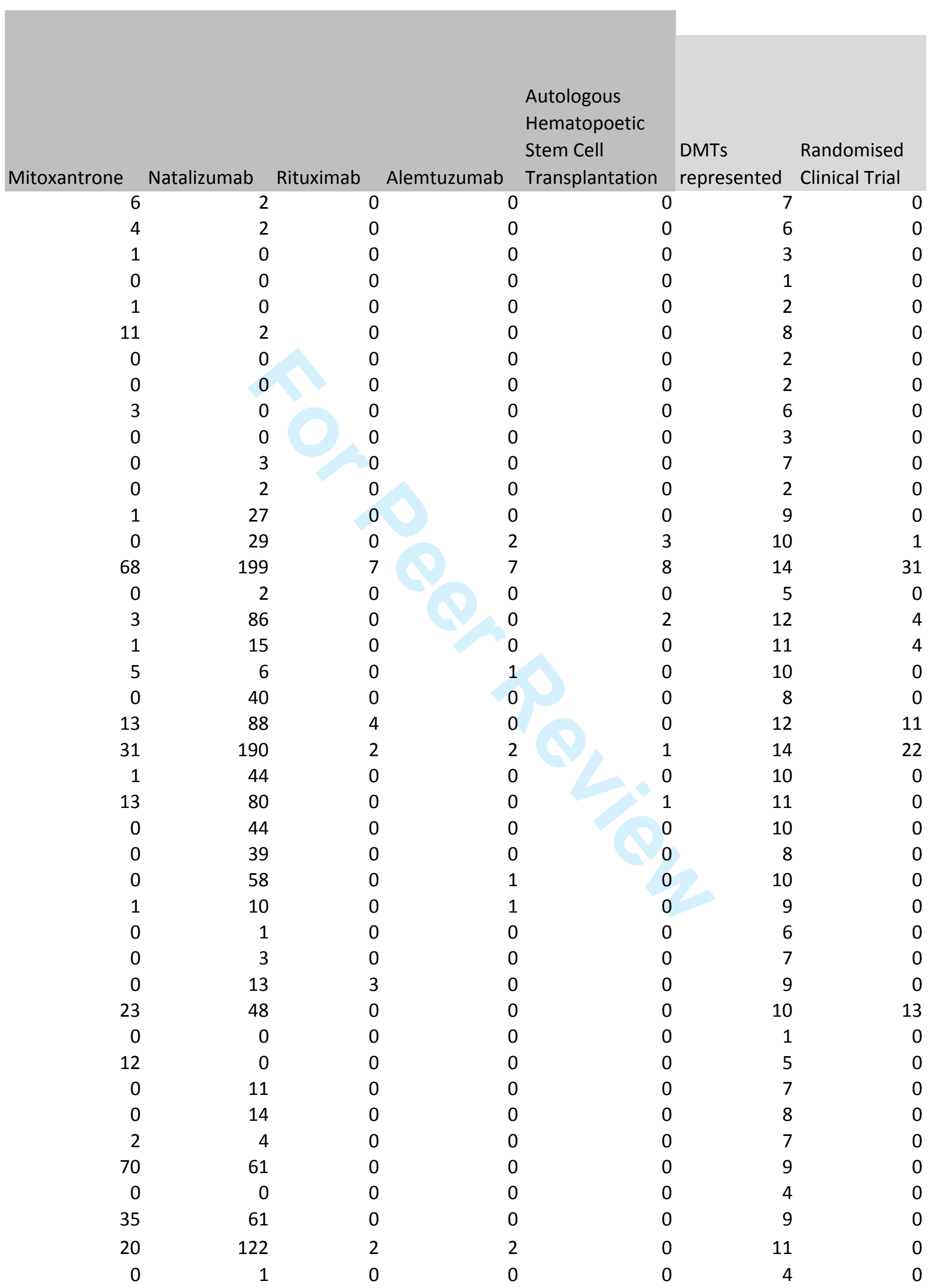




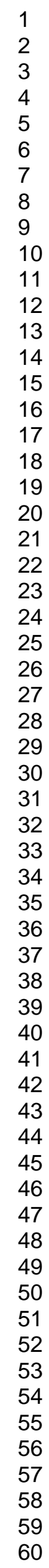

\begin{tabular}{|c|c|c|c|c|c|}
\hline 11 & 0 & 0 & 0 & 0 & 5 \\
\hline 32 & 381 & 36 & 11 & 17 & 13 \\
\hline 0 & 14 & 0 & 0 & 0 & 7 \\
\hline 57 & 0 & 0 & 0 & 0 & 5 \\
\hline 24 & 141 & 0 & 0 & 0 & 9 \\
\hline 0 & 0 & 0 & 0 & 0 & 0 \\
\hline 23 & 24 & 4 & 0 & 0 & 9 \\
\hline 7 & 19 & 2 & 0 & 0 & 9 \\
\hline 10 & 29 & 0 & 0 & 0 & 6 \\
\hline 0 & 12 & 1 & 0 & 0 & 8 \\
\hline 38 & 190 & 4 & 0 & 1 & 11 \\
\hline 0 & 0 & 0 & 0 & 0 & 0 \\
\hline 1 & 8 & 1 & 0 & 0 & 10 \\
\hline 5 & 24 & 1 & 1 & 0 & 11 \\
\hline 0 & 7 & 0 & 0 & 0 & 8 \\
\hline 1 & 0 & 0 & 0 & 0 & 5 \\
\hline 0 & 0 & 0 & 0 & 0 & 1 \\
\hline 0 & 1 & 0 & 0 & 0 & 5 \\
\hline 0 & 18 & 0 & 0 & 0 & 7 \\
\hline 0 & 10 & 0 & 0 & 0 & 8 \\
\hline 0 & 0 & 0 & 0 & 0 & 1 \\
\hline 0 & 0 & 0 & 0 & 0 & 0 \\
\hline 1 & 19 & 0 & 0 & 0 & 6 \\
\hline 1 & 76 & 0 & 0 & 0 & 6 \\
\hline 0 & 28 & 0 & 0 & 0 & 3 \\
\hline 0 & 24 & 0 & 0 & 0 & 6 \\
\hline 0 & 24 & 0 & 0 & 0 & 5 \\
\hline 0 & 18 & 0 & 0 & 0 & 5 \\
\hline 0 & 17 & 0 & 0 & 0 & 5 \\
\hline 0 & 13 & 0 & 0 & 0 & 3 \\
\hline 0 & 12 & 0 & 0 & 0 & 6 \\
\hline 0 & 6 & 0 & 0 & 0 & 3 \\
\hline 0 & 4 & 0 & 0 & 0 & 3 \\
\hline 0 & 6 & 0 & 0 & 0 & 6 \\
\hline 0 & 0 & 0 & 0 & 0 & 1 \\
\hline 1 & 0 & 0 & 0 & 0 & 5 \\
\hline 0 & 0 & 0 & 0 & 0 & 4 \\
\hline 24 & 1 & 5 & 0 & 0 & 7 \\
\hline 1 & 6 & 0 & 0 & 0 & 5 \\
\hline 93 & 0 & 0 & 0 & 0 & 6 \\
\hline 5 & 0 & 0 & 0 & 0 & 9 \\
\hline 0 & 0 & 0 & 0 & 0 & 2 \\
\hline 114 & 130 & 0 & 0 & 0 & 9 \\
\hline 30 & 35 & 1 & 0 & 1 & 11 \\
\hline 67 & 115 & 3 & 0 & 0 & 10 \\
\hline 19 & 3 & 0 & 0 & 0 & 9 \\
\hline 0 & 0 & 0 & 0 & 0 & 4 \\
\hline
\end{tabular}




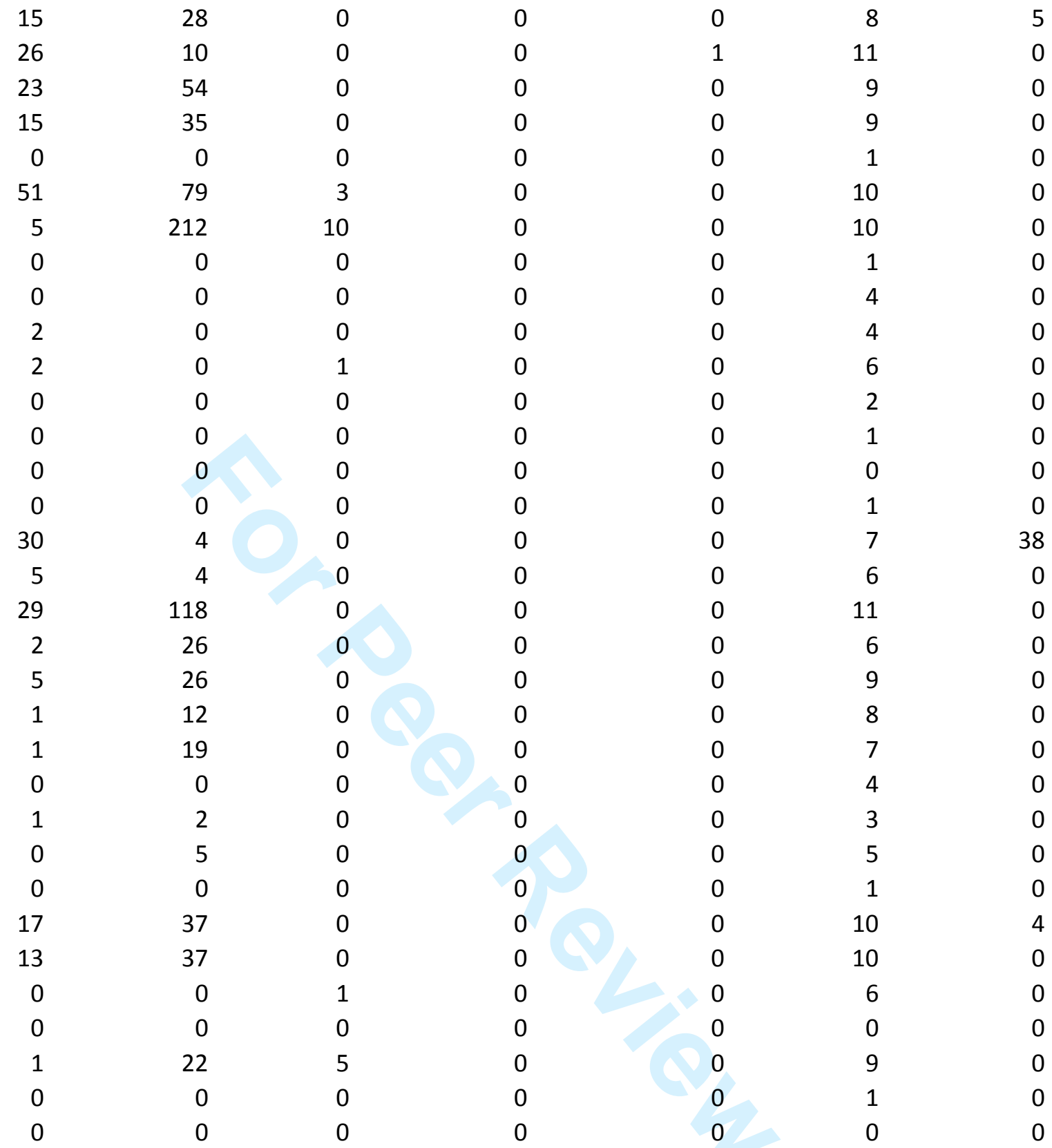




\begin{tabular}{|c|c|c|c|}
\hline $\begin{array}{l}\text { adverse } \\
\text { events } \\
\text { (count) }\end{array}$ & $\begin{array}{l}\% \text { females } \\
\text { pregnant }\end{array}$ & errors & $\begin{array}{l}\text { error rate } \\
\text { (per } 100 \\
\text { patient- } \\
\text { years) }\end{array}$ \\
\hline 0.08 & $17 \%$ & 232 & 52.2 \\
\hline 0.11 & $8 \%$ & 19 & 3.3 \\
\hline 0.06 & $0 \%$ & 11 & 22.4 \\
\hline 0.03 & $9 \%$ & 2 & 5.3 \\
\hline 0.00 & $0 \%$ & 4 & 0.0 \\
\hline 0.08 & $21 \%$ & 25 & 3.9 \\
\hline 0.24 & $42 \%$ & 8 & 24.4 \\
\hline 0.00 & $0 \%$ & 38 & 17.5 \\
\hline 0.00 & $0 \%$ & 58 & 6.1 \\
\hline 0.00 & $0 \%$ & 0 & 0.0 \\
\hline 0.02 & $0 \%$ & 3 & 3.2 \\
\hline 1.61 & $50 \%$ & 0 & 0.0 \\
\hline 0.01 & $2 \%$ & 11 & 6.2 \\
\hline 0.65 & $38 \%$ & 77 & 14.3 \\
\hline 0.10 & $29 \%$ & 131 & 2.8 \\
\hline 1.37 & $10 \%$ & 3 & 102.8 \\
\hline 0.63 & $18 \%$ & 39 & 1.8 \\
\hline 0.05 & $19 \%$ & 8 & 2.7 \\
\hline 0.23 & $28 \%$ & 45 & 12.5 \\
\hline 0.44 & $3 \%$ & 17 & 10.4 \\
\hline 0.28 & $22 \%$ & 19 & 1.3 \\
\hline 0.18 & $54 \%$ & 55 & 1.4 \\
\hline 0.24 & $32 \%$ & 48 & 6.5 \\
\hline 0.15 & $10 \%$ & 55 & 3.3 \\
\hline 0.02 & $37 \%$ & 41 & 3.0 \\
\hline 0.01 & $0 \%$ & 8 & 0.7 \\
\hline 0.46 & $7 \%$ & 44 & 19.5 \\
\hline 0.27 & $19 \%$ & 5 & 1.8 \\
\hline 0.11 & $0 \%$ & 1 & 5.4 \\
\hline 0.84 & $11 \%$ & 4 & 24.0 \\
\hline 0.05 & $34 \%$ & 18 & 7.9 \\
\hline 0.07 & $10 \%$ & 124 & 2.8 \\
\hline 0.00 & $100 \%$ & 0 & 0.0 \\
\hline 0.00 & $0 \%$ & 24 & 20.6 \\
\hline 0.03 & $2 \%$ & 6 & 2.6 \\
\hline 0.01 & $8 \%$ & 37 & 5.0 \\
\hline 0.00 & $16 \%$ & 6 & 1.5 \\
\hline 0.22 & $100 \%$ & 59 & 0.4 \\
\hline 0.29 & $0 \%$ & 7 & 20.6 \\
\hline 0.04 & $19 \%$ & 118 & 1.5 \\
\hline 0.01 & $8 \%$ & 92 & 1.4 \\
\hline 1.18 & $0 \%$ & 0 & 0.0 \\
\hline
\end{tabular}

http://mc.manuscriptcentral.com/multiple-sclerosis 


\begin{tabular}{|c|c|c|c|}
\hline 0.06 & $8 \%$ & 18 & 1.5 \\
\hline 0.02 & $24 \%$ & 251 & 1.0 \\
\hline 0.04 & $10 \%$ & 49 & 9.0 \\
\hline 0.00 & $0 \%$ & 5 & 145.4 \\
\hline 0.00 & $0 \%$ & 14 & 0.6 \\
\hline 0.00 & $4 \%$ & 2 & 0 \\
\hline 0.05 & $27 \%$ & 53 & 2. \\
\hline 0.05 & $35 \%$ & 24 & 2.6 \\
\hline 0.00 & $12 \%$ & 74 & 2.6 \\
\hline 0.10 & $18 \%$ & 17 & 3 \\
\hline 0.02 & $21 \%$ & 152 & \\
\hline 0.00 & $0 \%$ & 0 & 0.0 \\
\hline 0.08 & $14 \%$ & 18 & 2.6 \\
\hline 0.03 & $81 \%$ & 56 & \\
\hline 0.09 & $19 \%$ & 2 & 1.7 \\
\hline 0.01 & $2 \%$ & 15 & 11. \\
\hline 0.00 & $0 \%$ & 2 & 15.7 \\
\hline 0.00 & $1 \%$ & 13 & \\
\hline 0.18 & $50 \%$ & 13 & \\
\hline 0.15 & $14 \%$ & 16 & 8.4 \\
\hline 0.00 & $0 \%$ & 11 & 0.0 \\
\hline 0.00 & $13 \%$ & 7 & \\
\hline 0.26 & $20 \%$ & 1 & 1.6 \\
\hline 0.08 & $10 \%$ & 4 & \\
\hline 0.08 & $0 \%$ & 5 & 10.0 \\
\hline 0.25 & $27 \%$ & 5 & 2 \\
\hline 0.48 & $0 \%$ & 8 & 15.3 \\
\hline 0.15 & $18 \%$ & 7 & 26.6 \\
\hline 0.27 & $6 \%$ & 5 & 17 \\
\hline 0.18 & $0 \%$ & 2 & 7. \\
\hline 0.24 & $9 \%$ & 3 & 4. \\
\hline 0.80 & $0 \%$ & 0 & 0.0 \\
\hline 0.14 & $0 \%$ & 1 & 7. \\
\hline 0.16 & $0 \%$ & 3 & 7.8 \\
\hline 0.00 & $0 \%$ & 0 & 0.0 \\
\hline 0.00 & $0 \%$ & 1 & 2.5 \\
\hline 0.01 & $14 \%$ & 16 & 2 \\
\hline 0.02 & $0 \%$ & 234 & 41.5 \\
\hline 0.76 & $0 \%$ & 19 & 205.3 \\
\hline 0.00 & $0 \%$ & 94 & 5.2 \\
\hline 0.00 & $0 \%$ & 4 & 3.6 \\
\hline 0.00 & $0 \%$ & 0 & 0. \\
\hline 0.03 & $10 \%$ & 337 & 2.8 \\
\hline 0.10 & $26 \%$ & 60 & 1.7 \\
\hline 0.13 & $31 \%$ & 220 & 1. \\
\hline 0.19 & $12 \%$ & 22 & 1.7 \\
\hline 0.00 & $0 \%$ & 1 & 16. \\
\hline
\end{tabular}

http://mc.manuscriptcentral.com/multiple-sclerosis 


1
2
3
4
5
6
7
8
9
10
11
12
13
14
15
16
17
18
19
20
21
22
23
24
25
26
27
28
29
30
31
32
33
34
35
36
37
38
39
40
41
42
43
40
45
49
50
51
52
53
55
50

$\begin{array}{rrrr}0.04 & 4 \% & 42 & 1.0 \\ 0.13 & 6 \% & 28 & 1.0 \\ 0.06 & 18 \% & 58 & 3.0 \\ 0.01 & 16 \% & 48 & 2.7 \\ 0.00 & 0 \% & 0 & 0.0 \\ 0.04 & 16 \% & 140 & 2.1 \\ 0.01 & 7 \% & 42 & 2.2 \\ 0.00 & 0 \% & 6 & 84.2 \\ 0.00 & 11 \% & 103 & 48.2 \\ 0.06 & 0 \% & 7 & 2.4 \\ 0.00 & 5 \% & 0 & 0.0 \\ 0.88 & 0 \% & 12 & 70.7 \\ 0.00 & 7 \% & 0 & 0.0 \\ 3.04 & 0 \% & 0 & 0.0 \\ 0.00 & 0 \% & 0 & 0.0 \\ 0.00 & 4 \% & 18 & 0.5 \\ 0.00 & 23 \% & 22 & 8.8 \\ 0.04 & 25 \% & 161 & 2.7 \\ 0.00 & 1 \% & 4 & 1.6 \\ 0.03 & 21 \% & 85 & 5.5 \\ 0.02 & 18 \% & 78 & 30.5 \\ 0.05 & 24 \% & 102 & 4.6 \\ 0.00 & 0 \% & 1 & 17.3 \\ 0.01 & 0 \% & 7 & 4.4 \\ 0.47 & 7 \% & 16 & 23.3 \\ 0.00 & 0 \% & 0 & 0.0 \\ 0.06 & 90 \% & 305 & 8.6 \\ 0.12 & 63 \% & 85 & 2.5 \\ 0.11 & 31 \% & 4 & 2.0 \\ 0.00 & 0 \% & 0 & 0.0 \\ 0.06 & 0 \% & 4 & 2.3 \\ 0.00 & 0 \% & 0 & 0.0 \\ 0.00 & 0 \% & 2 & 0.0\end{array}$




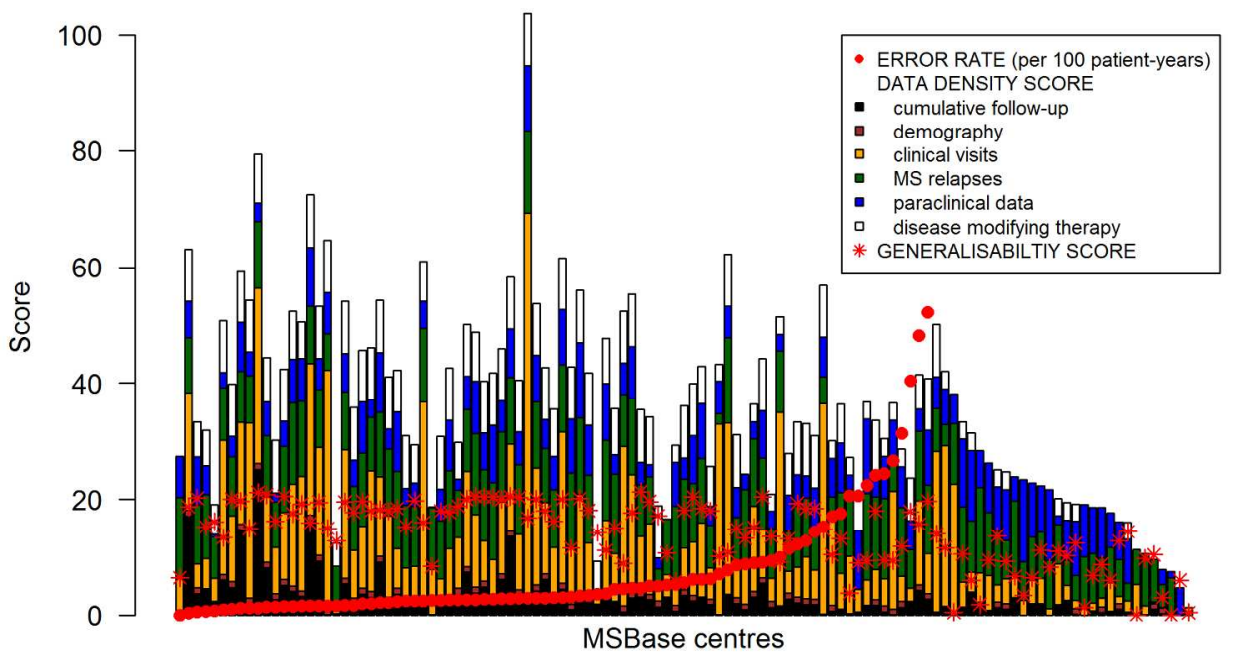

$199 \times 139 \mathrm{~mm}(300 \times 300 \mathrm{DPI})$ 


\section{University Library}

\section{- M M N E R VA A gateway to Melbourne's research publications}

Minerva Access is the Institutional Repository of The University of Melbourne

Author/s:

Kalincik, T;Kuhle, J;Pucci, E;Ignacio Rojas, J;Tsolaki, M;Sirbu, C-A;Slee, M;Butzkueven, H

Title:

Data quality evaluation for observational multiple sclerosis registries

Date:

2017-04-01

Citation:

Kalincik, T., Kuhle, J., Pucci, E., Ignacio Rojas, J., Tsolaki, M., Sirbu, C. -A., Slee, M. \& Butzkueven, H. (2017). Data quality evaluation for observational multiple sclerosis registries. MULTIPLE SCLEROSIS JOURNAL, 23 (5), pp.647-655. https:// doi.org/10.1177/1352458516662728.

Persistent Link:

http://hdl.handle.net/11343/127267 